\title{
Colored Corn: An Up-Date on Metabolites Extraction, Health Implication, and Potential Use
}

\author{
Raffaella Colombo $^{1}$ (D), Lucia Ferron ${ }^{1,2}$ and Adele Papetti ${ }^{1, *(D)}$ \\ 1 Department of Drug Sciences, University of Pavia, Viale Taramelli 12, 27100 Pavia, Italy; \\ raffaella.colombo@unipv.it (R.C.); lucia.ferron01@universitadipavia.it (L.F.) \\ 2 FlaNat Research Italia Srl, Via Giuseppe di Vittorio 1, 20017 Rho (Milano), Italy \\ * Correspondence: adele.papetti@unipv.it; Tel.: +39-0382-987-863
}

Citation: Colombo, R.; Ferron, L.;

Papetti, A. Colored Corn: An

Up-Date on Metabolites Extraction, Health Implication, and Potential Use. Molecules 2021, 26, 199. https:// doi.org/10.3390/molecules26010199

Academic Editor: Gianni Sacchetti Received: 12 November 2020 Accepted: 29 December 2020 Published: 2 January 2021

Publisher's Note: MDPI stays neutral with regard to jurisdictional clai$\mathrm{ms}$ in published maps and institutional affiliations.

Copyright: (C) 2021 by the authors. Licensee MDPI, Basel, Switzerland. This article is an open access article distributed under the terms and conditions of the Creative Commons Attribution (CC BY) license (https:// creativecommons.org/licenses/by/ $4.0 /)$.

\begin{abstract}
Colored (orange, pink, red, purple, and blue) corn strongly attracted attention on its healthy properties mainly due to its anthocyanin and carotenoid composition which is also responsible for its pigmentation. The present review summarized the recent updates on the extraction and chemical characterization of the main plant secondary metabolites present in colored seeds, kernel, cob, husk, and silk. The main approaches used to stabilize the extracts have been discussed as well as their food and non-food uses. Both in vitro and in vivo (animal models) studies on the different effects (antibacterial, antimutagenic, antioxidant, and anti-inflammatory activities, effects on metabolic syndrome, diabetes, glucose and lipidic metabolism, and neuroprotection) of pigmented extracts on animal and human health have been summarized.
\end{abstract}

Keywords: colored corn; purple corn; blue corn; corn waste; anthocyanins; polyphenols; extraction; corn-based food; non-food corn use

\section{Introduction}

One of the most produced cereal worldwide is Zea mays ssp. mays L. (corn) with about 1.15 billion tons estimated per year in 2018 [1]. Corn is commonly used for feed and food, but also for other different industrial purpose, i.e., pharmaceutical and cosmetic fields.

In the last decades, the scientific research focused the attention on pigmented corn varieties because of the well-known healthy properties of anthocyanins [2-6]. For this reason, breeding technologies have been pointed out to increase the anthocyanin content in uncolored varieties using different approaches, mainly starting from those traditionally cultivated in South and Central America [7]. Purple corn is typical of the Andean Region of South America where it has been used for centuries for the preparation of traditional food and beverages. Recently, it was also considered a good source of natural colorants to be used in textile industry [7] because of its content in anthocyanins; however, it also contained other phenolic compounds showing health-promoting effects. For these reasons, purple varieties attracted the attention of researchers to a greater extent for their potential use in food supplement and cosmetic industries. Anthocyanins are mainly located in pericarp in red and black varieties, while they are concentrated in the aleurone in blue corn [8].

The aim of this review is to summarize the results obtained in the last years about the biological properties tested both in vitro and in vivo, and the food and non-food use of pigmented corn varieties, considering not only kernels and its derived products, but also corn waste, i.e., husk and cob. In addition, different extraction approaches applied to obtain the tested extracts are discussed, as well as the different secondary metabolite profiles in relation to corn color.

\section{Pigmented Corns and By-Products}

Corn, also known as maize, is an ancient crop, nowadays still cultivated all around the world. Recently, it has been suggested that it was firstly domesticated as staple crop in 
Honduras around 4300 years ago, based on its discovery in the El Gigante rock shelter in the Honduran highlands [9]. Mythology reported that corn was kept inside a mountain, then divine intervention made it available to humanity by opening the mountain with a thunderbolt. However, the crashing thunder burned the corn, giving rise to the different kernel colors: the black seeds were the outermost, totally burnt, followed by blue, red, yellow and finally white ones, corresponding to the unburned seeds in the center of the mountain [7].

Ancient American corn varieties were reported to be widely pigmented, going from white to black colors. However, after the discovery of America in 1492 and the spread of this crop in Europe, it lost its pigmentation due to the adaptation process to European environmental conditions (longer photoperiod and cold-temperate climate), with only rare exceptions [7].

The corn color is mainly due to the presence of a huge number of secondary metabolites, such as phenolic acids, carotenoids, and flavonoids. The different expression of these pigments imparts to corn tissues different colors, from yellow-orange to dark purple-blue, as well as ivory, and cream colors. Pigments are generally mostly concentrated in the thick pericarp or in the aleurone layers of kernels, and also in corn cobs [10-12]. In plants, flavonoids physiological role consists in the recruitment of pollinators and protection of tissues from UV and oxidative stress. Several authors reported that the flavonoid biosynthesis pathways are modulated by two kind of transcription factors, encoded by the bHLH (basic Helix-Loop-Helix) and MYB (MYeloBlastosis) gene families, while WD40 (tryptophanaspartic acid 40) factor has been exclusively related to anthocyanins structural genes; the interaction of these transcription factors and their target genes resulted in both spatial and temporal biosynthesis of anthocyanins in maize [7,13-17].

Anthocyanins can be accumulated in seeds, corn stalks, cobs, and leaves and their different chemical structure together with their different contents lead to various color shades, from pink to dark blue, which characterized the different pigmented corn varieties.

However, it is well known that maize plant genotype is not the only factor determining corn pigmentation, as different environmental factors, altitude, temperature, and abiotic stress, in addition to maturation stage, could highly affect anthocyanins accumulation in corn $[18,19]$, differently from cultivation practices (for example, potassium fertilization and seeding density) which have less impact, as reported by Jing et al. for eighteen different purple corn cultivars from Perù [20]. The evaluation of the effect of location and genotype, in addition to their interactions, is useful for breeding, as demonstrated by Khampas et al. [21] who selected a distinct genotype grown in a particular zone of Thailand, characterized by high pelargonidin-3-O-glucoside and peonidin-3-O-glucoside levels and high 2,2-diphenyl-1-picrylhydrazyl (DPPH) radical scavenger capacity. Germination is another factor affecting polyphenols concentration, especially anthocyanins, as demonstrated by the decrease of their amount in purple corn seed, with the exception of peonidin malonylated forms [22].

Generally, purple corn kernels are richer in anthocyanin than blue $4910 \mathrm{mg}$ vs. $363 \mathrm{mg} / \mathrm{kg}$ dry corn) and red ones [12,18], and this content is very close to that of other food sources, but differently from these other sources it is concentrated in the pericarp and therefore easier extractable, thus it has been pointed out as an extremely valuable source of bioactive pigments [23].

Recently Li et al. [24] investigated the potential use of blue and purple corn byproducts obtained from both the dry- and wet-milling processes, showing that, based on the extent of anthocyanins accumulation in the aleurone layers and corn phenotype, highly pigmented coproduct suitable for further utilization could be obtained from these processes. Moreover, several studies reported that high content of pigments in corn were found in the inedible husk, cob, and silk, which ranged from $0.49 \%$ and $4.6 \%$ of the dry or fresh weight, respectively $[11,25,26]$. As regards corn silk, it is considered a waste product which could be used as natural colorant, flavoring agent or to produce value-added products such as snack, corn silk tea, or cosmetics. Different silk quality can be achieved according to the 
harvesting period: In fact, at silking stage it contains the highest amount of polyphenols, differently from what happened at the physiological maturity stage. At immature stage, an intermediate amount was detected [27]. Silk phytochemical composition was also evaluated for four purple waxy corn genotypes (Zea mays L. var. ceratina) at edible and seed stages by Simla et al. [28]. Corn, cob, and husk were also considered. Silk at both the maturity stages had higher content of anthocyanin and phenolics, as well as antiradical capacity against DPPH and 2,2'-azinobis [3-ethylbenzothiazoline-6-sulfonic acid]-diammonium salt (ABTS) than other ear components. Anyway, cob had a high anthocyanin content independently from the genotype and maturity stage. Considering that silk is used for the preparation of tea/herbal-like beverage, different colored silks (yellow, green, pink, and purple) were compared with six medicinal herbs for their polyphenols and chlorophyll contents. Corn silks at the silking stage were the richest in total polyphenols, with about two- to four-fold higher content than silks at the R4 dough stage, while anthocyanins highly contributed to the color of pink and purple silks and proanthocyanidins were present only in the purple colored variety. Moreover, their polyphenolic content is generally higher than that of Mentha piperita, Ginkgo biloba, Thymus serpyllum, and Salvia officinalis, but lower than that of green tea. HPLC-MS analyses indicated the presence of five chlorogenic acids, including 3-caffeoylquinic acid (-CQA), 4-CQA, 5-CQA, and $p$-coumaroylquinic acid, with 3-CQA and 4-CQA present in higher concentration (932.7-1840.8 mg/100 $\mathrm{g}$ and $186.9-426.6 \mathrm{mg}$ 3-CQA equivalents $/ 100 \mathrm{~g}$, respectively) and about two-fold content in purple and green silks with respect to yellow ones. Three flavone glycosides were also present, of which maysin and its methoxy analogue are prevalent. A lower chlorophyll $a$ and $b$ content than the medicinal herbs was found, and chlorophyll a was present in higher concentration than $\mathrm{b}$ in all the tested samples. Some minerals, i.e., $\mathrm{Na}, \mathrm{K}, \mathrm{Mg}$, and $\mathrm{Ca}$, were also quantified, with their concentration depending on silk maturation stage and only $\mathrm{K}$ level was higher at silking stage than at R4 dough stage. Moreover, as there is not a meaningful difference between corn silks at silking stage and the tested medicinal herbs and considering their polyphenolic profiles, corn silks could be used, similar to tea, as a functional ingredient in food supplements [29].

\section{Pigmented Maize All Around the World}

Anthocyanins rich corn varieties are mainly cultivated in South America, especially in Peru and Bolivia [20]. During these last years, several Central and South American pigmented corn accessions have been deeply investigated for their antocyanins content.

Several works reported that the 3-O-glycosylated (glucoside) forms of cyanidin, pelargonidin, and peonidin, along with their malonylated and succynil derivatives (i.e., cyanidin-3-O-(3"-Omalonylglucoside), cyanidin-3-O-(6"-O-malonylglucoside), pelargonidin-3-O-malonylglucoside, peonidin-3-O-malonylglucoside, cyanidin 3-O-(3", 6 "-O-dimalonylglucoside, cyanidin 3-O(6"-O-succinylglucoside), cyanidin 3-O-(3",6"-O-malonylsuccinylglucoside), and peonidin $3-O-\left(6^{\prime \prime}-\mathrm{O}\right.$-succinylglucoside) $)$ are the most common abundant and common compounds detected in these pigmented varieties [30,31]. Among them cianydin-3-O-glucoside (C3G) was showed to be mainly expressed in purple and red kernels, differently from its malonylated derivative which was revealed in higher concentration in red/blue and blue varieties [18,32]. Cyanidin-3-O-(6"-O-disuccinylglucoside) in addition to cyanidin-3-O-(6"$O$-succinylglucoside) was identified as a marker in fifteen blue corn accessions from the northwestern Mexico [33].

Moreover, González-Manzano et al. [34] reported the presence of flavanol-anthocyanin condensed pigment in two different native Mexican purple corn varieties, (cv. Arrocillo and cv. Peruano), in particular the adducts catechin-(4,8)-peonidin-3-glucoside, catechin(4,8)-cyanidin-3-glucoside, catechin-(4,8)-perlagonidin-3-glucoside, and their malonylated derivatives were identified through NMR analysis. These data were further confirmed by investigating Morado cultivar composition, the most widely diffused purple corn commercialized as a powder: it was shown to contain a particular flavonol-anthocyanin condensed pigment, namely catechin-(4,8)-cyanidin-3,5-O-diglucoside, previously found 
only in wine [35] and some fruits and vegetables [36]. This compound, identified by ${ }^{1} \mathrm{H}-$ and ${ }^{13} \mathrm{C}-\mathrm{NMR}$ using $1 \mathrm{D}$ and $2 \mathrm{D}$ techniques and by mass spectrometry, was also quantified in seed pericarp, in endosperm and in both parts of three varieties collected in Mexico, together with other cyanidin, peonidin or pelargonidin condensed pigments deriving from a C-C linkage between their $\mathrm{C} 8$ and $\mathrm{C} 4$ atoms in catechin or epicatechin. It was also noticed that these condensed compounds are not necessarily distributed in relation with the presence of the free anthocyanins, confirmed their naturally presence in some corn variety and not the fact that they formed during corn processing, as previously supposed [34]. Recently, the same molecules were identified in a particular Peruvian cultivar of purple corn (var. PMV-581), both in non-germinated and in germinated seeds [22]. A prevalence of pelargonidin-derived pigments, whose flavanol moiety was represented both by catechin or epicatechin and afzelechin, was highlighted in six different purple corn lines from the landrace Apache Red (AR), accounting for 10.1-18.9\% of the total anthocyanin content, differently from Morado purple corn in which they represented $28.3 \%$ of the content. Furthermore, AR lines are characterized by the presence of C-glycosyl flavones which could contribute to highlight the health bioactivities and the co-pigmentation, thus leading to further breeding programs to improve natural colorants concentration [37].

According to Collison et al. [18], purple, blue, red/blue, and red kernels of twenty-four different pigmented maize hybrids obtained from Peruvian Morado variety contained 890-3312, up to 540, up to 368 , and up to $127 \mathrm{mg}$ of C3G equivalent/g of dry matter, respectively, and a similar composition in anthocyanins was also registered for colored corn cob in addition to the presence of catechin-(4,8)-cyanidin-3,5-diglucoside [11].

Lower anthocyanins concentrations have been detected by spectrophotometric methods in four red and seven blue genotypes from Mexico (3.89-12.09 mg C3G equivalent $/ 100 \mathrm{~g}$ of dry matter for red samples and 10.24-34.17 mg C3G equivalent/100 $\mathrm{g}$ of dry matter for blue samples) [38]. Moreover, in all pigmented corn varieties, phenolic acids derivatives were also detected, as reported by Montilla et al. [39], who compared both anthocyanins and phenolic acids contents in ten different Bolivian purple corn cultivars. In this work the total content of phenolic acids were quantified using Folin-Ciocalteu assay showing that the sum of soluble and bound phenolics ranges from 311.0 to $817.6 \mathrm{mg}$ GAE/100 $\mathrm{g}$ of dry weight, while ferulic acid and $p$-coumaric acid were recognized as the most representative compounds of phenolic fraction present in all the varieties studied by HPLC-Diode Array Detectr (DAD) analysis. On the other hand, anthocyanin fraction was quantified in these ten samples by HPLC-DAD: the registered values were extremely different among the ten studied varieties, ranging from 1.97 and $71.68 \mathrm{mg}$ of C3G/100 g of dry weight. The cultivar Kulli showed the highest anthocyanins content, thus its composition was fully characterized, confirming that cyanidin-, perlagonidin-, and peonidin-glycosylated forms and their malonylate derivatives are the most representative in purple corn.

These data agree with the results reported by Urias-Lugo et al. [40], who compare total phenolic and anthocyanins content of two blue-corn varieties named Chalqueño and Conico with five hybrids, obtained from blue Mexican maize landraces and white varieties characterized by improved agronomic stability and adapted to highlands. Both the native varieties and the hybrids showed a similar composition: with a total anthocyanins content ranging from $646 \mathrm{mg}$ to $1052 \mathrm{mg}$ of C3G equivalent $/ \mathrm{kg}$, while the free and bound phenolic fractions content were 1037-1488 and 9064-11987 mg GAE/ kg, respectively.

Recently, two open-pollinated varieties deriving from Southwest and Corn Belt blue corn cultivated in different New Mexico locations, namely "Los Lunas High" and "Ohio Blue", were compared with six landrace accessions from different areas of Arizona and New Mexico for their content in anthocyanins as well as in amino acids, protein, starch, crude fiber, fatty acids, and ash. No significant differences among the tested samples were recorded and therefore they could be suitable for traditional dishes and their cultivation should be encouraged, considering also that the open-pollinated corn varieties were characterized by the presence of cyanidin-3-O-disuccynilglucoside [41,42]. 
Conversely, a high amount, i.e., $915.43 \pm 90.07 \mathrm{mg}$ C3G equivalent $/ \mathrm{kg}$, was found in a Turkish blue corn flour [43].

Considering European pigmented varieties, rare corn genotypes still display the anthocyanin colorations; among them "Millo Corvo" is an ancient landrace with dark blue/black kernels typically cultivated in Spain's region of Galicia, and it is particularly rich in anthocyanins $(83.45 \mathrm{mg} / 100 \mathrm{~g}$ of flours), flavonols $(74.21 \mathrm{mg} / 100 \mathrm{~g})$, and phenolic acids $(216.63 \mathrm{mg} / 100 \mathrm{~g})$, but it is poor in carotenoids [44]. In this context several Italian ancient pigmented varieties are reported, ranging from orange to dark purple.

Recently the genotype of pigmented "Rostrato Rosso" variety was investigated using an untargeted metabolomics approach based on Ultra-High Performance Liquid Chromatography with ElectroSpray Ionization, coupled to Quadrupole-Time-Of-Flight (UHPLCESI/QTOF) mass spectrometry, demonstrating that it is characterized by a particular phytocomplex, rich in both phenolic acids $(1149.7 \mathrm{mg} / \mathrm{kg})$, anthocyanins $(4399.4 \mathrm{mg} / \mathrm{kg})$, and other flavonols [45]. "Rostrato Rosso" mainly differs from South American pigmented maize for the presence of uncommon anthocyanins forms in pigmented corn, such as malvidin 3-O-(6"-acetyl-galactoside), or delphinidin 3-O-xyloside, and it is also rich in tyrosol and $\varepsilon$-carotene.

"Nero Spinoso" from Camonica valley in Italy, in turn, is an ancient cultivar characterized by the extremely dark black/red coloration mainly due to the high content of phlobaphenes, accumulated in the pericarp layer of seeds thank to a transcription factor belonging to the myb transcription factor gene family (Pericarp 1 (P1) gene). In addition, it contains also flavonoids and phenolic acids and all these peculiarities make this cultivar very interesting as functional food [46].

Finally, Capocchi et al. [47] chemically characterized four genotypes grown in the northern mountain zone of Tuscany whose kernel color ranged from orange to dark red. In red and orange varieties, the pigments accumulated in the pericarp and in the endosperm (carotenoids), respectively. High amount of phlobaphenes were detected in red grains which progressively decreased form dark red to yellow genotypes. High levels of carotenoids were also present in the endosperm of the red varieties with $\beta$-carotene being the less abundant, differently from zeaxanthin that is present in considerably higher amount, indicating a possible different biosynthetic pathway in these traditional genotypes. Ascorbic acid was present in a range of $10.33-64.89 \mathrm{mg} / \mathrm{g}$ flour with the lower content registered for the yellow variety. Based on these data, the traditional Tuscany corn varieties can be considered good source of nutraceutical molecules.

The cultivation of pigmented corn have been also reported from Asian countries: Khamphasan et al. [48] evaluated the effect of genotype and location, and their interaction, on the anthocyanin composition, total polyphenolic compounds, antioxidant activity, and color parameters of cob and husk from 53 different purple corns from Thailand, giving important information about the way to improve phytochemicals concentration in such corn by-products, which could be recycled for different purposes [48].

In 2016, Russian Research Scientific Institute of Corn published results related to new hybrid varieties with different pigmentation including a variety with grey tops and light yellow sides which had a similar protein content to that of the most conventionally used yellow corn and also a concentration of macro- and microelements very similar to that of a red variety; conversely, white and orange hybrid corn kernels had a higher content of starch and therefore they might be recommended for specific technological purposes. The maximum flavonoid content was registered in orange hybrid corn kernels ( $80 \mathrm{mg} / 100 \mathrm{~g})$, while the highest anthocyanin amount in rubin hybrid corn $(120 \mathrm{mg} / 100 \mathrm{~g})$ [49].

In Scheme 1, the main classes of compounds present in the different pigmented corn varieties are listed. 

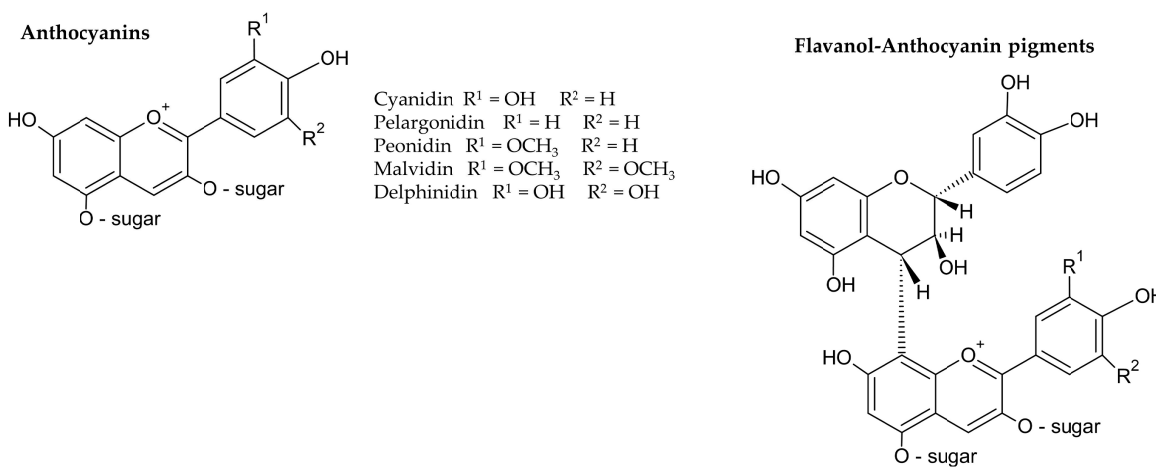

Catechin--(4,8)-anthocyanin mono/di-glucoside Cyanidin $\mathrm{R}^{1}=\mathrm{OH} \quad \mathrm{R}^{2}=\mathrm{H}$

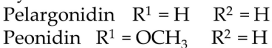

Hydroxycinnamic acid derivatives
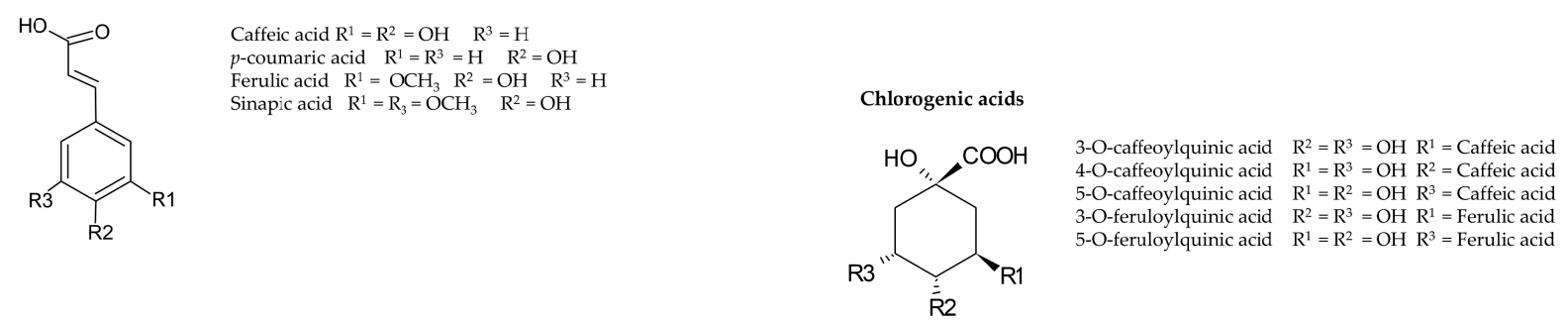

Benzoic acid derivatives<smiles>[R]c1cc(C(=O)O)cc(Br)c1[R]</smiles>

$p$-hydroxybenzoic acid $\mathrm{R}^{1}=\mathrm{R}^{2}=\mathrm{H} \quad \mathrm{R}^{3}=\mathrm{OH}$ Vanillic acid $\mathrm{R}^{1}=\mathrm{OCH}_{3} \quad \mathrm{R}^{2}=\mathrm{OH} \quad \mathrm{R}^{3}=\mathrm{H}$

R2<smiles>[R4]c1cc(O)c2c(=O)c([R3])c(-c3cc([R2])c(O)c([R2])c3)oc2c1</smiles>

Luteolin $\mathrm{R}^{1}=\mathrm{OH} \quad \mathrm{R}^{2}=\mathrm{H}^{3} \quad \mathrm{R}^{3}=\mathrm{HR}^{4}=\mathrm{OH}(\mathrm{O}$-sugar) Kaempferol $\quad \mathrm{R}^{1}=\mathrm{R}^{2}=\mathrm{H} \mathrm{R}^{3}=\mathrm{OH}(\mathrm{O}$ - sugar $) \mathrm{R}^{4}=\mathrm{OH}(\mathrm{O}$-sugar $)$ Quercetin $\mathrm{R}^{1}=\mathrm{OH} \quad \mathrm{R}^{2}=\mathrm{H} \mathrm{R}^{3}=\mathrm{OH}\left(\mathrm{O}\right.$ - sugar) $\mathrm{R}^{4}=\mathrm{OH}(\mathrm{O}$-sugar $)$ Myricetin $\mathrm{R}^{1}=\mathrm{R}^{2}=\mathrm{OH} \mathrm{R}^{3}=\mathrm{OH}(\mathrm{O}$ - sugar $) \mathrm{R}^{4}=\mathrm{OH}(\mathrm{O}$-sugar $)$ Isorhamnetin $\mathrm{R}^{1}=\mathrm{OCH}_{3} \quad \mathrm{R}^{2}=\mathrm{H} \mathrm{R}^{3}=\mathrm{OH}(\mathrm{O}$ - sugar $) \mathrm{R}^{4}=\mathrm{OH}(\mathrm{O}$-sugar $)$ Apigenin $\mathrm{R}^{1}=\mathrm{R}^{2}=\mathrm{H} \mathrm{R}^{3}=\mathrm{HR}^{4}=\mathrm{OH}$ (O-sugar)
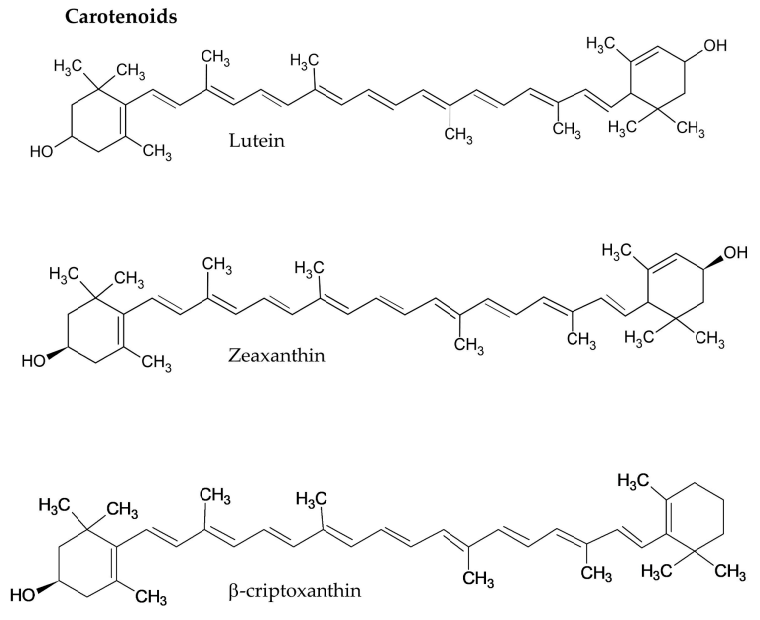<smiles>CC1=C(/C=C/C(C)=C/C=C/C(C)=C/C=C/C=C(C)/C=C/C=C(C)/C=C/C2=C(C)CCCC2(C)C)C(C)(C)CCC1</smiles>

Scheme 1. The main classes of compounds considered in this review are listed. 


\section{Factors Affecting Bioactive Extraction and Stability}

Secondary metabolites extraction from plant material is generally influenced by many intrinsic and extrinsic factors, from agricultural practice to pedo-climatic conditions, from extraction method to extraction conditions. Polyphenols and anthocyanins are easily affected by temperature, light exposure, and $\mathrm{pH}$ during storage and they tend to degrade into other products; therefore, many techniques over the years have been applied to enhance the stability as well as to optimize the yield of extraction.

The most common extraction procedures involved the use of aqueous-organic solvent mixture, generally acidified to improve the yield, at a temperature lower than $50-60{ }^{\circ} \mathrm{C}$, for different time [50]. An example is represented by the extraction of anthocyanins from purple corn cob using a food-friendly $50 \%$ aqueous ethanol acidified with $6 \mathrm{~N} \mathrm{HCl}(0.01 \%$, $v / v$ ) for $45 \mathrm{~min}$ and a double washing of the remaining colored insoluble powder after the extract filtration. This extract provided a high level of polyphenols and monomeric anthocyanins without chemically changing which could originate polymeric pigments [51]. However, in 2020 a new approach for water-based anthocyanin recovery from purple corn was investigated. A single-stage, two-stage, three-stage, and two-stage countercurrent water-based extractions resulted in a recovery of $48.6,68.6,77.9$, and $66.8 \%$, respectively. Moreover, a three-stage process was techno-economic feasible for commercial scale application [52].

An improvement in the extraction was generally achieved by ultrasound-assisted extraction by using $95 \%$ ethanol with $0.1 \mathrm{M}$ citric acid, and setting the experimental conditions as follows: ultrasonic times 90 , power $400 \mathrm{~W}$, and solid:liquid ratio 1:8. This set up was then successfully scaled-up for the extraction of C3G (45\% total anthocyanins) and cyanidin-3-O(6"-O-malonylglucoside) ( $40 \%$ of total anthocyanins) from purple bran. Lower percentage of pelargonidin-3-O-glucoside, pelargonidin-3-O-(6"-O-malonylglucoside), peonidin-3$O$-glucoside, and cyanidin-3-O-(3",6"-di-O-malonylglucoside) were also obtained [53]. Conversely, a higher content of malonylated (mono- and di-) than non-malonylated cyanidin derivatives was registered for the husk using similar conditions [25]. Different operative conditions were applied to a Mexican red corn cob. Solid:liquid ratio and extraction time highly affected the yield of bioactives which increased from 10 to 30 solid:liquid ratio (w:v) and from 60 to $120 \mathrm{~min}$ of extraction time (from $215.17 \pm 33.49$ to $527.33 \pm 103.79 \mathrm{GAE}$ $\mathrm{mg} / 100 \mathrm{~g} \mathrm{DW})$. The use of ethanol $55 \%$ allowed the extraction of 5-CQA, $p$-coumaric acid and caffeic acid 4-O-hexoside, in addition to three glycosylated apigenin (hexoside, pentosyl hexoside, and 6-C-pentosyl-8-C-hexoside), luteolin-O-rutinoside, and scopoletin [54]. Ultrasound-assisted extraction was also the most efficient procedure to extract anthocyanins (1:10 solvent-to-solid ratio, ethanol/water/lactic acid mixture, 80:19:1, for $20 \mathrm{~min}$, $100 \mathrm{~W}$ ) from Cahuacintle corn and husk. Additionally, an enzymatic-assisted extraction using xylanases, celluclast, and depol, was used since the residual material obtained after the extraction exhibited an intense color. The optimized $\mathrm{pH}$ and temperature conditions gave an extract richer in cyanidin-3-O-(6"-O-malonyl)glucoside than the maceration or microwave-assisted extraction methods [55].

Oxidation of polyphenols should be prevented during extraction and the most used method consists in performing this process under $\mathrm{N}_{2}$ purging, thus leading to high yield of extraction. In the case of anthocyanins extraction from kernel and cob, applying $0.20 \mathrm{MPa}$ for 15 min under $\mathrm{N}_{2}$ purging, $0.492 \mathrm{mg}$ and $1.890 \mathrm{mg}$ C3G equivalents/g of dry kernel and cob, respectively, were extracted at $80^{\circ} \mathrm{C}$ by using sample:solvent ratio $1: 8$ and water:ethanol ratio 1:3 for kernel and 1:1 for cob. This operative condition together with the solvent mixture markedly suitable for anthocyanin polarity promoted the swelling of sample, thus enhancing the contact area between sample and solvent. Other extraction process conditions were studied, but the above reported were identified as the best to avoid both impurity dissolution and anthocyanin degradation which could be at higher temperature, as well as at longer time of extraction [56]. Ohmic heating extraction is another interesting approach that could be used; it was applied to a new purple corn single cross-hybrid from Thailand and the yield of total anthocyanins extraction was compared to that obtained 
by using other extraction methods based on the use of water as solvent and operating in optimized conditions (conventional extraction applied for $5 \mathrm{~min}$ at $70^{\circ} \mathrm{C}$, ultrasoundassisted extraction for $5 \mathrm{~min}$ at $80^{\circ} \mathrm{C}, 37 \mathrm{KHz}$, and microwave-assisted extraction for $2 \mathrm{~min}$, $2.45 \mathrm{GHz}, 700 \mathrm{~W}$ ). This new approach is based on the rapid change of electrical energy into heat which is able to generate a uniform temperature distribution through the sample solution submitted to the extraction. It has two main advantages: it does not affect the nutritional properties differently from conventional extraction and leads to higher yield in a less time. However, it produces the lowest amount of anthocyanins in comparison with the other methods, and also, the color of the extract is darker, probably due to a quick overheating of cob powder which burnt producing carbon. Microwave-assisted extraction was the best method, and it was scaled-up giving a total anthocyanin yield of $0.25 \%$ and producing a dark purple powder arising a deep red colored solution after the dissolution in water [57]. Another interesting extraction method, used to maximize the amount of anthocyanins to be used as dye in food, was a steeping step of the pericarp in sodium metabisulfite generating $\mathrm{SO}_{2}$ in presence of lactic acid which facilitates faster $\mathrm{SO}_{2}$ sorption. $\mathrm{SO}_{2}$ should then promote the pigments extraction by a possible interaction of anthocyanins with $\mathrm{HSO}_{3}{ }^{-}$leading to a better diffusion through cell walls and thus to an increased solubility of pigments. Moreover, lactic acid lowered $\mathrm{pH}$ of the solution, enhancing anthocyanin extraction and stability. This treatment allowed the extraction of $22.9 \pm 0.2 \mathrm{mg}$ C $3 \mathrm{G}$ monomeric anthocyanin equivalents/g dry pericarp and also of the condensed anthocyanins, differently from the extraction with only water in which the condensed forms were prevalent with only $7.1 \pm 0.6 \mathrm{mg}$ C3G/g dry pericarp, or steeping water with $0.2 \% \mathrm{SO}_{2}$, which extracted only monomeric anthocyanins, but in a lower amount $(20.5 \pm 1.5 \mathrm{mg}$ C $3 \mathrm{G} / \mathrm{g}$ dry pericarp). Flavonoids concentration did not differ in the different approaches [58]. The foliar part of corn could also be a source of anthocyanins. Its content in a new dark red corn variety was evaluated using a quick, simple and conservative method, namely hyperspectral model, generally used for measuring moisture, nitrogen, and chlorophyll content. To overcome the weak relationship between the anthocyanin content and the hyperspectrum, a sensitive band $(685 \mathrm{~nm})$ was selected via multiple linear regression thus enhancing the accuracy and the stability of the model, also when the anthocyanin content was lower than $20 \mathrm{mg} / \mathrm{g}$ [59].

High-pressure processing for 30-45 min differently affected purple waxy corn kernel polyphenols contents and antioxidant properties depending on the pressure applied. Pressure treatment at $700 \mathrm{MPa}$ was the best condition to have the highest yields in phytochemicals with a color profile overlapping to that of steam-treated kernels. Conversely, lower pressure (from 250 up to $550 \mathrm{MPa}$ ) reduced the recovery of polyphenols with a progressively reduction as the pressure decreased. This different action of pressure treatment is probably correlated with the cell rupture and the subsequent release of polyphenols from food materials occurring at the highest pressure level (700 MPa). Moreover, this treatment promoted starch gelatinization with a slower retrogradation than a generic thermal cooking process. Even if high pressure processing is generally not suitable for foods rich in water soluble compounds, the results obtained in this work supported its potential use in the development of new healthy products [60].

Despite the, relatively, easy use of all these methods, the set-up of raw material:solvent ratio, solvent mixture composition, time, and temperature of extraction are time consuming but not so expensive. A great advantage could derive by the use of experimental design. One of the most recently used approach is response surface methodology (RSM). Its application allowed Hwang et al. [61] optimizing solid:solvent ratio, solvent volume, extraction time, and temperature, performing only few experiments to set up the best conditions (at $40{ }^{\circ} \mathrm{C}$ for $8 \mathrm{~h}$, with 1:15 in solvent volume, and a $33 \%$ solid:liquid ratio) to extract the highest amount of the 3-O-glycosidic derivatives of cyanidin, pelargonidin, peonidin, and the malonylglycosidic derivatives of cyanidin and peonidin from a commercially purple corn kernel. In these conditions, the best yield extraction was also obtained for other polyphenols, such as hyrsutrin and its methoxylated derivative, hydroxy benzoic $(2,4,6-$ trihydroxy benzoic, protocatechuic, and vanillic acids) and hydroxycinnamic (ferulic and 
p-hydroxycinnamic acids) acid derivatives. By using this approach, the same anthocyanins in addition to pelargonidin-3-O-(6"-O-malonyl)-glucoside, malvidin-3-O-(6"-O-malonyl- $p$ hydroxybenzoyl)-glucoside, and malvidin-3-O-(6"-O-dimalonyl)-glucoside were extracted by a purple corn produced in China, without any degradation. RSM was also adopted to optimize the ultrasound-assisted extraction of both anthocyanins and zein (a prolamine protein representing about $40 \%$ of total protein content, widely used in different industrial fields for its peculiar characteristics). The results obtained were based on single-factor experiments which considered solid/liquid ratio, solvent extraction composition (ethanol percentage), and power, time, and temperature as ultrasound parameters. The highest yield in anthocyanins $(450 \mathrm{mg} / \mathrm{kg}$ corn) was obtained using a 1:26 solid (g)/liquid (mL) ratio, $74 \%$ ethanol $(v / v)$ at $70{ }^{\circ} \mathrm{C}$ for $90 \mathrm{~min}$, with an ultrasound power of $105 \mathrm{~W}$, after different purification steps consisting in a precipitation with ammonium sulphate precipitation, followed by the separation from zein using a cross-flow ultrafiltration, and a final purification by AB- 8 macroporous resins [62]. In another research, a central composite rotatable design (CCRD) was applied to study the effects of two parameters, i.e., temperature and pressure, on many other variables, namely yield of extraction (obtained at each of the three steps applied by sequentially using supercritical carbon dioxide, ethanol, and water), total content of phenolics, flavonoids, and monomeric anthocyanins, anti-DPPH radical capacity and CIE $\mathrm{L}^{*} \mathrm{a}^{*} \mathrm{~b}^{*}$ system. Using RSM, the best process conditions for purple corn cob extraction were detected in the use of ethanol both at $65^{\circ} \mathrm{C}$ and 450 bar for the extraction of total phenolics (389 $\mathrm{mg}$ gallic acid equivalents/g extract) and antiradical activity, and at $45^{\circ} \mathrm{C}$ and 420 bar for the extraction of total monomeric anthocyanins (64 mg C3G equivalents/g extract of which 26-38 mg/g were C3G); conversely, the highest yield of extraction for total flavonoids was obtained using water at $50{ }^{\circ} \mathrm{C}$ and $400 \mathrm{bar}(93.7 \mathrm{mg}$ catechin equivalents/g) [31].

Purple corn cob could be a good and economic source of anthocyanins with its general content higher than kernel, as demonstrated by Lao and Giusti [51]. Thus, it could represent a relatively cheap material for the extraction of natural colorant, even if the cereal texture complexed with other compounds present in cob made more difficult the optimization of the extraction procedure. Muangrat et al. [63] successfully used the central composite face-centered design to develop a RSM predicting the effect of ultrasound amplitude levels and different solvent extraction mixture on total anthocyanin and polyphenol levels. Waterethanol 1:1, amplitude level of $50 \%$, at $65^{\circ} \mathrm{C}$ for $30 \mathrm{~min}$ with a sample:solvent ratio of 1:30 were the best conditions identified to extract $27.66 \mathrm{mg}$ and $0.240 \mathrm{mg}$ of gallic acid and C3G equivalents/g dry sample, respectively. The use of ultrasound-assisted extraction has two main advantages: the acceleration of the swelling of cells accompanied by the fragmentation of the tissue matrix whose particles are hydrated by water present in the extraction solvent mixture and thus the penetration of the organic solvent component is facilitated, as well as the intensification of mass transfer by diffusion. Eight main anthocyanins were identified in the extract: cyanidin-3-O-(6"-O-ethylmalonylglucoside) and petunidin-3-O-glucoside in addition to C3G, pelargonidin-3-O-glucoside, peonidin-3$O$-glucoside, and their malonyl derivatives.

All these recent examples of application of RSM to different types of corn and corn waste confirmed the high potential of this approach and its help in the reduction of time and solvent/reagent consumption.

In the last decade the demand of natural colorants increased day by day in order to replace synthetic substances, considered less safe for food purposes, and therefore, stability and color studies became mandatory. Anthocyanins is one of the most studied class of natural colorants together with anthraquinones, betalains, and carotenoids. One of the main issues regarding the use of colored corn extract is its stability. In fact, anthocyanins are red-purple colored in acidic conditions, and following the deprotonation and quinoidal form generation, due to $\mathrm{pH}$ increase, they generate a purple-blue color; moreover, metal chelation and co-pigmentation can generate a blue color even in acidic conditions. Other factors influencing the rate of degradation include oxygen, light, temperature, and, also, 
enzymes. Metal chelation transformed flavylium cations into blue quinoidal structure and these complexes were stabilized by acylation on anthocyanin skeleton [64]. These chelates increased the stability of anthocyanins also during storage and heat treatment even if an excess of metals could degrade these pigments [65]. Anthocyanin chromophores can interact with colorless molecules or intramolecularly with acyl residues on the same anthocyanin by hydrophobic or $\pi-\pi$ linkages, thus, generating a blue co-pigment [66]. Recently, a systematic evaluation of the role of acylation in the formation of intramolecular co-pigments was investigated using $\mathrm{Al}^{3+}$ and $\mathrm{Fe}^{3+}$ as metal ions and performing the experiments at $\mathrm{pH}$ 6-7 in the dark and at room temperature for $48 \mathrm{~h}$. The anthocyanins isolated and purified both from a purple corn and a red cabbage extract changed their color more markedly in the case of chelation of $\mathrm{Fe}^{3+}$; moreover, at $\mathrm{pH} 6$ these changes were less evident starting from diacylated cyanidin to non-acylated derivatives. An improvement in pigment stability was registered with acylation increasing and among mono- and diacylated derivative the stability was in the order malonic $>$ sinapic $>$ ferulic $>p$-coumaric and $p$ coumaric-sinapic $>$ ferulic-sinapic $>$ sinapic-sinapic, respectively [67]. The addition of ferulic acid at different concentration $(4,8$, and $12 \mathrm{mg} / \mathrm{mL}$ extract) to a purple corn cob extract obtained by a $24 \mathrm{~h}$ maceration of $100 \mathrm{~g}$ of powder in $400 \mathrm{~mL}$ methanol in the dark at $4{ }^{\circ} \mathrm{C}$ determined the co-pigmentation of the antochyanin monoglucoside present in the extract (C3G, peonidin-3-O-glucoside, and pelargonidin-3-O-glucoside), thus originating the corresponding vinylguaiacol adducts which provided a color-enhancing effect, as demonstrated by CIE L*a*b* color space parameters. Moreover, a stabilizing effect was highlighted when the extract was stored for 90 days at $25^{\circ} \mathrm{C}$ in the dark [68].

In the past several researchers studied the stability of corn anthocyanins pointing out the greater stability of red/blue with respect to purple/red compounds also during a thermo-alkaline process such as nixtamalization [69]. More recently, the stability of anthocyanin extracts obtained from purple corn pericarp using an accelerated solvent extraction system was evaluated at different $\mathrm{pH}$ storage conditions for 12 weeks at $22^{\circ} \mathrm{C}$. The color degradation and chemical stability as the $\mathrm{pH}$ increased were confirmed also in this research: in fact, the color differences measured with CIE $\mathrm{L}^{*} \mathrm{a}^{*} \mathrm{~b}^{*}$ moved from 0.2 to 3.6 and from 17.7 to 47.5 during 12 weeks of storage, at $\mathrm{pH} 2$ and 6, respectively; moreover, the predicted half-life of total anthocyanins ranged from 44.6 to 60.7 weeks and from 1.8 to 3 weeks, at $\mathrm{pH} 2$ and 6, respectively. Flavanol-anthocyanin condensed forms or pyranoanthocyanins showed degradation kinetics similar to that of monomeric anthocyanins. Based on the obtained acceptable shelf-life data, the authors stated that the pigments extracted from purple corn pericarp can be used as natural colorant in acid beverages [70].

In order to stabilize the anthocyanin extracts different approaches based on complexation and co-pigmentation have been evaluated in the last years using Arabic gum or whey proteins or polysaccharides, in addition to metal ions. For example, in 2017 Luna-Vital et al. [71] studied the effect of alginate and zinc on color and chemical stability of purple corn water soluble pericarp extract in a beverage model. Using an experimental design, 13 different samples prepared using a solution of commercially available colorless Jammers cherry flavor (Kool-Aid Invisible ${ }^{\circledR}, 70.8 \mathrm{~g} / \mathrm{L}$ ) added to extract alone (final concentration $0.5 \mathrm{mg} \mathrm{DW} / \mathrm{mL}$ ) or to extract plus alginate $(0.01 \%)$ or to extract plus alginate and $\mathrm{ZnCl}_{2}$ at different concentrations were tested. Thermal stability was evaluated at 70, 80 , and $90^{\circ} \mathrm{C}$ up to $6 \mathrm{~h}$ and shelf-life at $25^{\circ} \mathrm{C}$ in the dark for 12 weeks. Zinc and alginate reduced thermal and shelf-life degradation through the formation of a complex, differently from alginate alone which improved stability only at $70^{\circ} \mathrm{C}$. The Stern-Volmer fluorescence quenching approach was used to assess the complex formation which indicated that the chemical interaction between the fluorophore (anthocyanin extract) and the quencher ( $\mathrm{ZnCl}_{2}$ or alginate) was due to static quenching, thus slowing the chemical degradation of extract. The results were very promising as both alginate and zinc were used in a lower concentration than the limits which could alter flavor and therefore, could be considered good additives to improve anthocyanin extracts stability. Maltodextrin was also evaluated 
as carrier in the production of spry-dried colored corn cob extract. Extraction solvents and purification step and drying inlet temperature, in addition to different amount of carrier $(2 \%, 5 \%$, and $10 \%)$ were key factors. In particular, inlet temperature highly affected water solubility of the final product and $150{ }^{\circ} \mathrm{C}$ coupled with the use of $5 \%$ maltodextrin resulted in a $90 \%$ of pigment yield, highly soluble with the least color loss [72].

Encapsulation is another approach to improve stability and the use of biopolymerbased hydrogels is essential in the case of food matrices. In the last years, anthocyanin extracts isolated from different matrices have been encapsulated in Ca-alginate hydrogels obtained by incorporation of calcium ions to form the gel matrix [73,74]. In 2018 Guo et al. [75] performed a study evaluating the effect of alginate to pectin ratio, total hydrocolloid concentration, and $\mathrm{pH}$ on encapsulation efficiency and stability of anthocyanins from purple corn when exposed to light and temperature. The encapsulation efficiency of hydrogel during manufacturing improved from $26 \%$ to $65 \%$ by increasing curing bath, but also alginate to pectin ratio and total gum concentration can highly impact this too. Another important result consisted in the improvement of stability of extract in hydrogel spherical particles applying low temperature and high particle weight:solution volume ratio. Finally, the half-life values following a first-order kinetics degradation increased with encapsulation ( $630 \mathrm{~h}$ vs. $58 \mathrm{~h}$ of anthocyanin aqueous solution).

Recently, the use of microencapsulation by spray drying has been successfully adopted; in particular, a modified beta-cyclodextrin, hydroxypropyl beta-cyclodextrin, was used as a coating agent able to bind anthocyanins in a more effective way, thus improving the core compounds stability. The thus obtained purple corn powder extract was stable both at $4{ }^{\circ} \mathrm{C}$ and $30^{\circ} \mathrm{C}$ for 60 days of storage, even if the best storage condition for peonidin3-O-glucoside and pelargonidin-3-O-glucoside was $4{ }^{\circ} \mathrm{C}$. At this temperature, a lower increase in the concentration of protocatechuic and hydroxybenzoic acids, deriving from the degradation of anthocyanidin at B-ring level, was registered [76]. Interesting studies were performed on nine different corn extracts obtained by an accelerated solvent extraction system on unique pigmented corn collected by the University of Illinois. The extracts have been added to a colorless cherry flavor beverage model and stored at different temperatures $\left(4{ }^{\circ} \mathrm{C}, 22{ }^{\circ} \mathrm{C}\right.$, and $\left.32{ }^{\circ} \mathrm{C}\right)$ for 12 weeks. The beverages richest in $\mathrm{C} 3 \mathrm{G}$ and condensed anthocyanins maintained the color for a longer time at $32{ }^{\circ} \mathrm{C}$ during the entire monitoring time, differently from those containing a high concentration of peonidin derivatives [77].

In Table 1, secondary metabolites of colored corn (kernel, cob, and flour and derived products) in addition to the extraction solvent and method used for their detection are reported.

Table 1. Secondary metabolites of colored corn (kernel, cob, and flour and derived products). If not specified, the extraction method consisted in a solid/liquid extraction method.

\begin{tabular}{|c|c|c|c|}
\hline Compound & Sample & Extraction Solvent & Reference \\
\hline Cyanidin & Purple corn pericarp & $\mathrm{H}_{2} \mathrm{O}$, Accelerated Solvent Extraction & [77] \\
\hline \multirow[t]{8}{*}{ Cyanidin-3-O-glucoside } & Purple corn cob & Acidified $(0.01 \% \mathrm{HCl} 6 \mathrm{~N}) 70 \%$ acetone & {$[11,20]$} \\
\hline & & Acidified ( $1 \%$ citric acid) $\mathrm{MeOH}$ & [21] \\
\hline & & $\mathrm{MeOH}$ & [30] \\
\hline & & Three-sequential step: Supercritical & [31] \\
\hline & & $\begin{array}{c}\mathrm{CO}_{2}, \mathrm{EtOH} \text {, water } \\
\text { Acidified }\end{array}$ & \\
\hline & & $\begin{array}{l}\text { Acidified }(0.01 \% \mathrm{HCl} 6 \mathrm{~N}) 70 \% \text { acetone } \\
\text { or } \mathrm{MeOH} \text { or } \mathrm{H}_{2} \mathrm{O} \text { or } 20 \% \mathrm{EtOH} \text { or } 40 \%\end{array}$ & \\
\hline & & $\begin{array}{c}\mathrm{EtOH} \text { or } 50 \% \mathrm{EtOH} \text { or } 60 \% \mathrm{EtOH} \text { or } \\
80 \% \mathrm{EtOH} \text { or } \mathrm{EtOH}\end{array}$ & [51] \\
\hline & & $\begin{array}{l}60-100 \% \text { EtOH, } w / w, \text { ultrasound } \\
\text { assisted extraction }\end{array}$ & [62] \\
\hline
\end{tabular}


Table 1. Cont

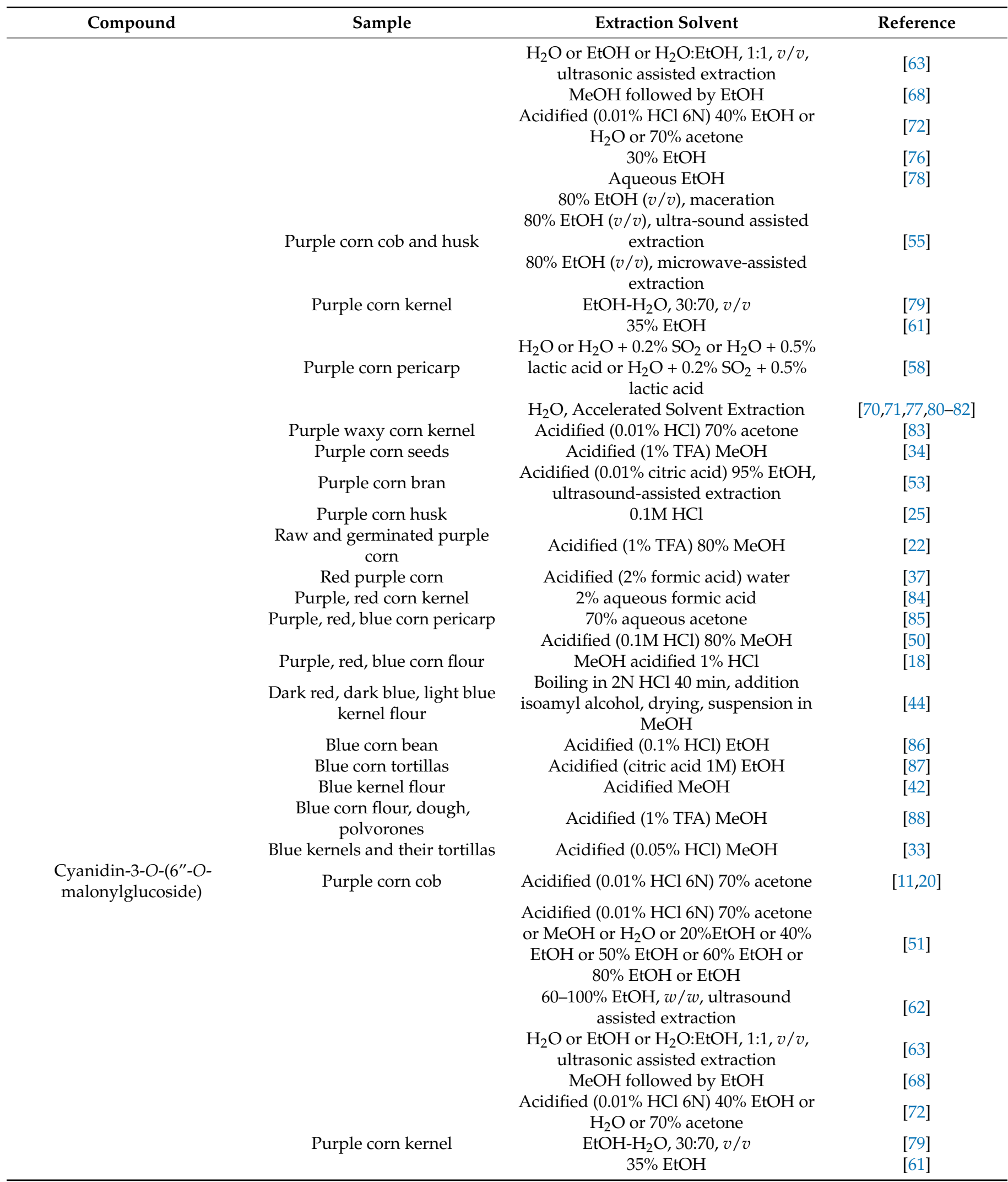


Table 1. Cont.

\begin{tabular}{|c|c|c|c|}
\hline Compound & Sample & Extraction Solvent & Reference \\
\hline & Purple corn pericarp & $\begin{array}{c}\mathrm{H}_{2} \mathrm{O} \text { or } \mathrm{H}_{2} \mathrm{O}+0.2 \% \mathrm{SO}_{2} \text { or } \mathrm{H}_{2} \mathrm{O}+0.5 \% \\
\text { lactic acid or } \mathrm{H}_{2} \mathrm{O}+0.2 \% \mathrm{SO}_{2}+0.5 \% \\
\text { lactic acid }\end{array}$ & [58] \\
\hline & & $\mathrm{H}_{2} \mathrm{O}$, Accelerated Solvent Extraction & {$[70,71,77,81,82]$} \\
\hline & Purple corn seeds & Acidified (1\% TFA) MeOH & {$[34]$} \\
\hline & Purple corn bran & $\begin{array}{c}\text { Acidified (0.01\% citric acid) } 95 \% \mathrm{EtOH}, \\
\text { ultrasound-assisted extraction }\end{array}$ & [53] \\
\hline & Purple corn husk & $0.1 \mathrm{M} \mathrm{HCl}$ & [25] \\
\hline & $\begin{array}{c}\text { Raw and germinated purple } \\
\text { corn }\end{array}$ & Acidified (1\% TFA) $80 \% \mathrm{MeOH}$ & [22] \\
\hline & Red purple corn & Acidified ( $2 \%$ formic acid) water & [37] \\
\hline & Purple, red corn kernel & $2 \%$ aqueous formic acid & [84] \\
\hline & Purple, red, blue corn flour & $\mathrm{MeOH}$ acidified $1 \% \mathrm{HCl}$ & [18] \\
\hline & Purple, red, blue corn pericarp & Acidified $(0.1 \mathrm{M} \mathrm{HCl}) 80 \% \mathrm{MeOH}$ & [50] \\
\hline & Red, blue corn pericarp & $70 \%$ aqueous acetone & [85] \\
\hline & Blue corn bean & Acidified $(0.1 \% \mathrm{HCl}) \mathrm{EtOH}$ & [86] \\
\hline & Blue corn tortillas & Acidified (citric acid 1M) EtOH & [87] \\
\hline & Blue kernels and their tortillas & Acidified $(0.05 \% \mathrm{HCl}) \mathrm{MeOH}$ & [33] \\
\hline & $\begin{array}{l}\text { Blue corn flour, dough, } \\
\text { polvorones }\end{array}$ & Acidified (1\% TFA) MeOH & {$[88]$} \\
\hline & Corn cob and husk & $\begin{array}{c}80 \% \mathrm{EtOH}(v / v) \text {, maceration } \\
80 \% \mathrm{EtOH}(v / v), \text { ultra-sound assisted } \\
\text { extraction } \\
80 \% \mathrm{EtOH}(v / v), \text { microwave-assisted } \\
\text { extraction }\end{array}$ & [55] \\
\hline \multirow{4}{*}{$\begin{array}{l}\text { Cyanidin-3-O-(3"-O- } \\
\text { malonylglucoside) }\end{array}$} & Purple corn cob & Acidified $(0.01 \% \mathrm{HCl}) 70 \%$ acetone & {$[20]$} \\
\hline & Purple corn flour & $\mathrm{MeOH}$ acidified $1 \% \mathrm{HCl}$ & [18] \\
\hline & Purple corn husk & $0.1 \mathrm{M} \mathrm{HCl}$ & [25] \\
\hline & Blue kernels and their tortillas & Acidified $(0.05 \% \mathrm{HCl}) \mathrm{MeOH}$ & [33] \\
\hline \multirow{2}{*}{$\begin{array}{l}\text { Cyandin-3-O-(6"-O- } \\
\text { ethylmalonylglucoside) }\end{array}$} & Purple corn cob & $\begin{array}{l}\mathrm{H}_{2} \mathrm{O} \text { or EtOH or } \mathrm{H}_{2} \mathrm{O}: \text { EtOH, } 1: 1, v / v \\
\text { ultrasonic assisted extraction }\end{array}$ & {$[63]$} \\
\hline & Blue corn tortillas & Acidified (citric acid 1M) EtOH & [87] \\
\hline $\begin{array}{l}\text { Cyanidin-3-O-(6"- } \\
\text { acetylgalactoside) }\end{array}$ & Blue corn tortillas & Acidified (citric acid 1M) EtOH & [87] \\
\hline $\begin{array}{l}\text { Cyanidin-3-O-(6"- } \\
\text { piruvoylglucoside) }\end{array}$ & Blue corn tortillas & Acidified (citric acid 1M) EtOH & [87] \\
\hline $\begin{array}{l}\text { Cyanidin-3-O-glucoside } \\
\text { monomalonate }\end{array}$ & Purple corn husk & $0.1 \mathrm{M} \mathrm{HCl}$ & [25] \\
\hline $\begin{array}{l}\text { Cyanidin-3-O- } \\
\text { malonylhexoside }\end{array}$ & $\begin{array}{l}\text { Raw and germinated purple } \\
\text { corn }\end{array}$ & Acidified (1\% TFA) $80 \% \mathrm{MeOH}$ & [22] \\
\hline \multirow{7}{*}{$\begin{array}{l}\text { Cyanidin-3-O-(3"-6"- } \\
\text { dimalonylglucoside) }\end{array}$} & Blue kernels and their tortillas & Acidified $(0.05 \% \mathrm{HCl}) \mathrm{MeOH}$ & [33] \\
\hline & Purple corn cob & Acidified $(0.01 \% \mathrm{HCl}) 70 \%$ acetone & {$[20]$} \\
\hline & Purple corn pericarp & $\mathrm{H}_{2} \mathrm{O}$, Accelerated Solvent Extraction & [77] \\
\hline & Purple corn husk & $0.1 \mathrm{M} \mathrm{HCl}$ & [25] \\
\hline & $\begin{array}{c}\text { Raw and germinated purple } \\
\text { corn }\end{array}$ & Acidified (1\% TFA) $80 \% \mathrm{MeOH}$ & [22] \\
\hline & Purple, red, blue corn pericarp & Acidified $(0.1 \mathrm{M} \mathrm{HCl}) 80 \% \mathrm{MeOH}$ & [50] \\
\hline & $\begin{array}{l}\text { Blue corn flour, dough, } \\
\text { polvorones }\end{array}$ & Acidified (1\% TFA) MeOH & {$[88]$} \\
\hline $\begin{array}{l}\text { Cyanidin-3-O-glucoside- } \\
\text { dimalonate }\end{array}$ & Purple corn husk & $0.1 \mathrm{M} \mathrm{HCl}$ & [25] \\
\hline
\end{tabular}


Table 1. Cont.

\begin{tabular}{|c|c|c|c|}
\hline Compound & Sample & Extraction Solvent & Reference \\
\hline \multirow{4}{*}{$\begin{array}{l}\text { Cyanidin-3-O-(6"'- } \\
\text { succinylglucoside) }\end{array}$} & Purple corn cob & Acidified $(0.01 \% \mathrm{HCl} 6 \mathrm{~N}) 70 \%$ acetone & [11] \\
\hline & Blue corn bean & Acidified $(0.1 \% \mathrm{HCl}) \mathrm{EtOH}$ & [86] \\
\hline & Blue kernels and their tortillas & Acidified $(0.05 \% \mathrm{HCl}) \mathrm{MeOH}$ & [33] \\
\hline & Blue kernel flour & Acidified $\mathrm{MeOH}$ & [42] \\
\hline $\begin{array}{l}\text { Cyanidin-3-O-(6"- } \\
\text { caffeoilglucoside) }\end{array}$ & Blue corn bean & Acidified $(0.1 \% \mathrm{HCl}) \mathrm{EtOH}$ & {$[86]$} \\
\hline \multirow{2}{*}{$\begin{array}{l}\text { Cyanidin-3-O-(6"- } \\
\text { disuccinylglucoside) }\end{array}$} & Blue kernel flour & Acidified $\mathrm{MeOH}$ & [42] \\
\hline & Blue kernels and their tortillas & Acidified $(0.05 \% \mathrm{HCl}) \mathrm{MeOH}$ & [33] \\
\hline \multirow[t]{3}{*}{ Cyanidin-3,5-diglucoside } & $\begin{array}{c}\text { Raw and germinated purple } \\
\text { corn }\end{array}$ & Acidified (1\% TFA) $80 \% \mathrm{MeOH}$ & [22] \\
\hline & Red purple corn & Acidified ( $2 \%$ formic acid) water & [37] \\
\hline & Blue corn tortillas & Acidified (citric acid 1M) EtOH & [87] \\
\hline \multirow[t]{27}{*}{ Pelargonidin-3-O-glucoside } & Purple corn cob & Acidified $(0.01 \% \mathrm{HCl} 6 \mathrm{~N}) 70 \%$ acetone & {$[11,20]$} \\
\hline & & Acidified (1\% citric acid) $\mathrm{MeOH}$ & [21] \\
\hline & & $\mathrm{MeOH}$ & {$[30]$} \\
\hline & & $\begin{array}{l}\text { Three-sequential step: Supercritical } \\
\qquad \mathrm{CO}_{2}, \mathrm{EtOH} \text {, water }\end{array}$ & {$[31]$} \\
\hline & & $\begin{array}{c}\text { Acidified }(0.01 \% \mathrm{HCl} 6 \mathrm{~N}) 70 \% \text { acetone } \\
\text { or } \mathrm{MeOH} \text { or } \mathrm{H}_{2} \mathrm{O} \text { or } 20 \% \mathrm{EtOH} \text { or } 40 \% \\
\text { EtOH or } 50 \% \mathrm{EtOH} \text { or } 60 \% \mathrm{EtOH} \text { or } \\
80 \% \mathrm{EtOH} \text { or } \mathrm{EtOH}\end{array}$ & [51] \\
\hline & & $\begin{array}{l}\mathrm{H}_{2} \mathrm{O} \text { or EtOH or } \mathrm{H}_{2} \mathrm{O}: \mathrm{EtOH}, 1: 1, v / v \\
\text { ultrasonic assisted extraction }\end{array}$ & {$[63]$} \\
\hline & & $\mathrm{MeOH}$ followed by EtOH & [68] \\
\hline & & $\begin{array}{l}\text { Acidified }(0.01 \% \mathrm{HCl} 6 \mathrm{~N}) 40 \% \mathrm{EtOH} \text { or } \\
\qquad \mathrm{H}_{2} \mathrm{O} \text { or } 70 \% \text { acetone }\end{array}$ & {$[72]$} \\
\hline & & $30 \% \mathrm{EtOH}$ & [76] \\
\hline & & Aqueous EtOH & [78] \\
\hline & & $\begin{array}{l}60-100 \% \text { EtOH, } w / w \text {, ultrasound } \\
\text { assisted extraction }\end{array}$ & {$[62]$} \\
\hline & & $\mathrm{MeOH}$ followed by EtOH & [68] \\
\hline & & $80 \% \mathrm{EtOH}(v / v)$, maceration & \\
\hline & Purple corn cob and husk & $\begin{array}{c}80 \% \operatorname{EtOH}(v / v), \text { ultra-sound assisted } \\
\text { extraction } \\
80 \% \operatorname{EtOH}(v / v), \text { microwave-assisted } \\
\text { extraction }\end{array}$ & [55] \\
\hline & Purple corn kernel & $\mathrm{EtOH}-\mathrm{H}_{2} \mathrm{O}, 30: 70, v / v$ & [79] \\
\hline & & $35 \% \mathrm{EtOH}$ & [61] \\
\hline & Purple corn pericarp & $\begin{array}{c}\mathrm{H}_{2} \mathrm{O} \text { or } \mathrm{H}_{2} \mathrm{O}+0.2 \% \mathrm{SO}_{2} \text { or } \mathrm{H}_{2} \mathrm{O}+0.5 \% \\
\text { lactic acid or } \mathrm{H}_{2} \mathrm{O}+0.2 \% \mathrm{SO}_{2}+0.5 \% \\
\text { lactic acid }\end{array}$ & [58] \\
\hline & & $\mathrm{H}_{2} \mathrm{O}$, Accelerated Solvent Extraction & {$[70,71,77,80-82]$} \\
\hline & Purple corn seeds & Acidified (1\% TFA) $\mathrm{MeOH}$ & [34] \\
\hline & Purple corn bran & $\begin{array}{c}\text { Acidified (0.01\% citric acid) } 95 \% \mathrm{EtOH} \\
\text { ultrasound-assisted extraction }\end{array}$ & {$[53]$} \\
\hline & Purple corn husk & $0.1 \mathrm{M} \mathrm{HCl}$ & [25] \\
\hline & Purple corn flour & $\mathrm{MeOH}$ acidified $1 \% \mathrm{HCl}$ & [18] \\
\hline & $\begin{array}{l}\text { Raw and germinated purple } \\
\text { corn }\end{array}$ & Acidified (1\% TFA) $80 \% \mathrm{MeOH}$ & [22] \\
\hline & Purple, red corn pericarp & $70 \%$ aqueous acetone & [85] \\
\hline & Purple, red corn kernel & $2 \%$ aqueous formic acid & [84] \\
\hline & Purple, red, blue corn pericarp & Acidified (0.1M HCl) $80 \% \mathrm{MeOH}$ & [50] \\
\hline & Purple waxy corn kernel & Acidified $(0.01 \% \mathrm{HCl}) 70 \%$ acetone & [83] \\
\hline
\end{tabular}


Table 1. Cont.

\begin{tabular}{|c|c|c|c|}
\hline Compound & Sample & Extraction Solvent & Reference \\
\hline & $\begin{array}{l}\text { Dark red, dark blue, light blue } \\
\text { kernel flour }\end{array}$ & $\begin{array}{c}\text { Boiling in } \mathrm{HCl} 2 \mathrm{~N} 40 \mathrm{~min} \text {, addition } \\
\text { isoamyl alcohol, drying, suspension in } \\
\text { MeOH }\end{array}$ & [44] \\
\hline & Blue corn bean & Acidified $(0.1 \% \mathrm{HCl}) \mathrm{EtOH}$ & [86] \\
\hline & Blue kernel flour & Acidified $\mathrm{MeOH}$ & [42] \\
\hline & Blue kernels and their tortillas & Acidified $(0.05 \% \mathrm{HCl}) \mathrm{MeOH}$ & [33] \\
\hline & $\begin{array}{l}\text { Blue corn flour, dough, } \\
\text { polvorones }\end{array}$ & Acidified (1\% TFA) MeOH & {$[88]$} \\
\hline \multirow[t]{24}{*}{$\begin{array}{l}\text { Pelargonidin-3-O-(6"- } \\
\text { malonylglucoside) }\end{array}$} & \multirow[t]{3}{*}{ Purple corn cob } & Acidified $(0.01 \% \mathrm{HCl} 6 \mathrm{~N}) 70 \%$ acetone & [11] \\
\hline & & Acidified $(0.01 \% \mathrm{HCl}) 70 \%$ acetone & [20] \\
\hline & & $\begin{array}{c}\mathrm{H}_{2} \mathrm{O} \text { or } \mathrm{EtOH} \text { or } \mathrm{H}_{2} \mathrm{O}: \mathrm{EtOH}, 1: 1, v / v \\
\text { ultrasonic assisted extraction }\end{array}$ & [63] \\
\hline & \multirow{3}{*}{$\begin{array}{l}\text { Purple corn flour } \\
\text { Raw and germinated purple } \\
\text { corn } \\
\text { Blue kernels and their tortillas }\end{array}$} & $\mathrm{MeOH}$ acidified $1 \% \mathrm{HCl}$ & [18] \\
\hline & & Acidified (1\% TFA) $80 \% \mathrm{MeOH}$ & [22] \\
\hline & & & \\
\hline & Purple corn seeds & Acidified (1\% TFA) MeOH & [34] \\
\hline & Red purple corn & $2 \%$ aqueous formic acid & {$[37]$} \\
\hline & Purple corn cob & $\begin{array}{c}\text { Acidified }(0.01 \% \mathrm{HCl} 6 \mathrm{~N}) 70 \% \text { acetone } \\
\text { or } \mathrm{MeOH} \text { or } \mathrm{H}_{2} \mathrm{O} \text { or } 20 \% \mathrm{EtOH} \text { or } 40 \% \\
\text { EtOH or } 50 \% \mathrm{EtOH} \text { or } 60 \% \mathrm{EtOH} \text { or } \\
80 \% \mathrm{EtOH} \text { or } \mathrm{EtOH}\end{array}$ & {$[51]$} \\
\hline & Purple corn bran & $\begin{array}{c}\text { Acidified (0.01\% citric acid) } 95 \% \text { EtOH, } \\
\text { ultrasound-assisted extraction }\end{array}$ & [53] \\
\hline & Purple corn husk & $0.1 \mathrm{M} \mathrm{HCl}$ & [25] \\
\hline & Purple corn pericarp & $\begin{array}{c}\mathrm{H}_{2} \mathrm{O} \text { or } \mathrm{H}_{2} \mathrm{O}+0.2 \% \mathrm{SO}_{2} \text { or } \mathrm{H}_{2} \mathrm{O}+0.5 \% \\
\text { lactic acid or } \mathrm{H}_{2} \mathrm{O}+0.2 \% \mathrm{SO}_{2}+0.5 \% \\
\text { lactic acid }\end{array}$ & [58] \\
\hline & Purple corn kernel & $35 \% \mathrm{EtOH}$ & {$[61]$} \\
\hline & Purple corn & $\begin{array}{c}60-100 \% \text { EtOH, } w / w, \text { ultrasound } \\
\text { assisted extraction }\end{array}$ & {$[62]$} \\
\hline & \multirow{3}{*}{ Purple corn cob and husk } & $80 \%$ EtOH $(v / v)$, maceration & \multirow{3}{*}{ [55] } \\
\hline & & $\begin{array}{c}80 \% \text { EtOH }(v / v), \text { ultra-sound assisted } \\
\text { extraction }\end{array}$ & \\
\hline & & $\begin{array}{c}80 \% \mathrm{EtOH}(v / v), \text { microwave-assisted } \\
\text { extraction }\end{array}$ & \\
\hline & Purple corn pericarp & $\mathrm{H}_{2} \mathrm{O}$, Accelerated Solvent Extraction & {$[70,71,77,81,82]$} \\
\hline & Purple corn cob & $\begin{array}{l}\text { Acidified }(0.01 \% \mathrm{HCl} 6 \mathrm{~N}) 40 \% \mathrm{EtOH} \text { or } \\
\qquad \mathrm{H}_{2} \mathrm{O} \text { or } 70 \% \text { acetone }\end{array}$ & [72] \\
\hline & Blue corn bean & Acidified $(0.1 \% \mathrm{HCl}) \mathrm{EtOH}$ & [86] \\
\hline & \multirow[t]{2}{*}{ Purple, red, blue corn pericarp } & $70 \%$ aqueous acetone & [85] \\
\hline & & Acidified $(0.1 \mathrm{M} \mathrm{HCl}) 80 \% \mathrm{MeOH}$ & {$[50]$} \\
\hline & \multirow{2}{*}{$\begin{array}{l}\text { Purple, red corn kernel } \\
\text { Blue corn flour, dough, } \\
\text { polvorones }\end{array}$} & $2 \%$ aqueous formic acid & {$[84]$} \\
\hline & & Acidified (1\% TFA) MeOH & [88] \\
\hline $\begin{array}{l}\text { Pelargonidin-3-O-(3"- } \\
\text { malonylglucoside) }\end{array}$ & Purple corn husk & $0.1 \mathrm{M} \mathrm{HCl}$ & [25] \\
\hline \multirow{4}{*}{$\begin{array}{l}\text { Pelargonidin-3-O-(3"-6"- } \\
\text { dimalonylglucoside) }\end{array}$} & Purple corn seeds & Acidified (1\% TFA) MeOH & {$[34]$} \\
\hline & \multirow{3}{*}{$\begin{array}{c}\text { Purple corn husk } \\
\text { Purple, red, blue corn pericarp } \\
\text { Raw and germinated purple } \\
\text { corn }\end{array}$} & $0.1 \mathrm{M} \mathrm{HCl}$ & [25] \\
\hline & & Acidified $(0.1 \mathrm{M} \mathrm{HCl}) 80 \% \mathrm{MeOH}$ & [50] \\
\hline & & Acidified (1\% TFA) $80 \% \mathrm{MeOH}$ & [22] \\
\hline
\end{tabular}


Table 1. Cont.

\begin{tabular}{|c|c|c|c|}
\hline Compound & Sample & Extraction Solvent & Reference \\
\hline Pelargonidin-3,5-diglucoside & Red purple corn & $2 \%$ aqueous formic acid & [37] \\
\hline \multirow[t]{34}{*}{ Peonidin-3-O-glucoside } & \multirow[t]{13}{*}{ Purple corn cob } & Acidified $(0.01 \% \mathrm{HCl} 6 \mathrm{~N}) 70 \%$ acetone & {$[11,20]$} \\
\hline & & Acidified (1\% citric acid) $\mathrm{MeOH}$ & {$[21]$} \\
\hline & & $\mathrm{MeOH}$ & {$[30]$} \\
\hline & & Three-sequential step: Supercritical & [31] \\
\hline & & $\begin{array}{l}\qquad \mathrm{CO}_{2}, \mathrm{EtOH}, \text { water } \\
\text { Acidified }(0.01 \% \mathrm{HCl} 6 \mathrm{~N}) 70 \% \text { acetone }\end{array}$ & \\
\hline & & or $\mathrm{MeOH}$ or $\mathrm{H}_{2} \mathrm{O}$ or $20 \% \mathrm{EtOH}$ or $40 \%$ & [51] \\
\hline & & $\begin{array}{c}\text { EtOH or } 50 \% \mathrm{EtOH} \text { or } 60 \% \mathrm{EtOH} \text { or } \\
80 \% \mathrm{EtOH} \text { or } \mathrm{EtOH}\end{array}$ & \\
\hline & & $\begin{array}{c}60-100 \% \mathrm{EtOH}, w / w, \text { ultrasound } \\
\text { assisted extraction }\end{array}$ & [62] \\
\hline & & $\begin{array}{c}\mathrm{H}_{2} \mathrm{O} \text { or EtOH or } \mathrm{H}_{2} \mathrm{O}: \mathrm{EtOH}, 1: 1, v / v \\
\text { ultrasonic assisted extraction }\end{array}$ & {$[63]$} \\
\hline & & $\mathrm{MeOH}$ followed by EtOH & [68] \\
\hline & & $\begin{array}{c}\text { Acidified }(0.01 \% \mathrm{HCl} 6 \mathrm{~N}) 40 \% \mathrm{EtOH} \text { or } \\
\mathrm{H}_{2} \mathrm{O} \text { or } 70 \% \text { acetone }\end{array}$ & {$[72]$} \\
\hline & & $30 \% \mathrm{EtOH}$ & [76] \\
\hline & & Aqueous EtOH & [78] \\
\hline & \multirow[t]{3}{*}{ Purple corn kernel } & $\mathrm{EtOH}-\mathrm{H}_{2} \mathrm{O}, 30: 70, v / v$ & [79] \\
\hline & & $35 \% \mathrm{EtOH}$ & [61] \\
\hline & & $\mathrm{H}_{2} \mathrm{O}$ or $\mathrm{H}_{2} \mathrm{O}+0.2 \% \mathrm{SO}_{2}$ or $\mathrm{H}_{2} \mathrm{O}+0.5 \%$ & \\
\hline & \multirow[t]{2}{*}{ Purple corn pericarp } & $\begin{array}{c}\text { lactic acid or } \mathrm{H}_{2} \mathrm{O}+0.2 \% \mathrm{SO}_{2}+0.5 \% \\
\text { lactic acid }\end{array}$ & [58] \\
\hline & & $\mathrm{H}_{2} \mathrm{O}$, Accelerated Solvent Extraction & {$[70,71,77,80-82]$} \\
\hline & Purple corn seeds & Acidified (1\% TFA) MeOH & [34] \\
\hline & Purple corn bran & $\begin{array}{c}\text { Acidified }(0.01 \% \text { citric acid) } 95 \% \mathrm{EtOH}, \\
\text { ultrasound-assisted extraction }\end{array}$ & [53] \\
\hline & Purple corn husk & $0.1 \mathrm{M} \mathrm{HCl}$ & [25] \\
\hline & \multirow{2}{*}{$\begin{array}{c}\text { Purple corn flour } \\
\text { Raw and germinated purple } \\
\text { corn }\end{array}$} & $\mathrm{MeOH}$ acidified $1 \% \mathrm{HCl}$ & [18] \\
\hline & & Acidified (1\% TFA) $80 \% \mathrm{MeOH}$ & {$[22]$} \\
\hline & Purple waxy corn kernel & Acidified $(0.01 \% \mathrm{HCl}) 70 \%$ acetone & [83] \\
\hline & Red purple corn & $2 \%$ aqueous formic acid & [37] \\
\hline & Purple, red corn kernel & $2 \%$ aqueous formic acid & [84] \\
\hline & Purple, red, blue corn pericarp & $70 \%$ aqueous acetone & [89] \\
\hline & & Acidified $(0.1 \mathrm{M} \mathrm{HCl}) 80 \% \mathrm{MeOH}$ & [50] \\
\hline & $\begin{array}{c}\text { Dark red, dark blue, light blue } \\
\text { kernel flour }\end{array}$ & $\begin{array}{l}\text { Boiling in } \mathrm{HCl} 2 \mathrm{~N} \text {, addition isoamyl } \\
\text { alcohol, drying, suspension in } \mathrm{MeOH}\end{array}$ & {$[44]$} \\
\hline & Blue kernel flour & Acidified $\mathrm{MeOH}$ & [42] \\
\hline & & $80 \% \operatorname{EtOH}(v / v)$, maceration & \\
\hline & & $80 \% \mathrm{EtOH}(v / v)$, ultra-sound assisted & \\
\hline & Corn cob and husk & extraction & [55] \\
\hline & & $\begin{array}{c}80 \% \text { EtOH }(v / v), \text { microwave-assisted } \\
\text { extraction }\end{array}$ & \\
\hline \multirow[t]{6}{*}{$\begin{array}{l}\text { Peonidin-3-O-(6"- } \\
\text { malonylglucoside) }\end{array}$} & \multirow[t]{6}{*}{ Purple corn cob } & Acidified $(0.01 \% \mathrm{HCl} 6 \mathrm{~N}) 70 \%$ acetone & {$[11,20]$} \\
\hline & & $\begin{array}{c}\text { Acidified }(0.01 \% \mathrm{HCl} 6 \mathrm{~N}) 70 \% \text { acetone } \\
\text { or } \mathrm{MeOH} \text { or } \mathrm{H}_{2} \mathrm{O} \text { or } 20 \% \mathrm{EtOH} \text { or } 40 \% \\
\text { EtOH or } 50 \% \mathrm{EtOH} \text { or } 60 \% \mathrm{EtOH} \text { or } \\
80 \% \mathrm{EtOH} \text { or } \mathrm{EtOH}\end{array}$ & {$[51]$} \\
\hline & & $\begin{array}{c}60-100 \% \mathrm{EtOH}, w / w, \text { ultrasound } \\
\text { assisted extraction }\end{array}$ & [62] \\
\hline & & $\begin{array}{l}\mathrm{H}_{2} \mathrm{O} \text { or } \mathrm{EtOH} \text { or } \mathrm{H}_{2} \mathrm{O}: \mathrm{EtOH}, 1: 1, v / v \\
\text { ultrasonic assisted extraction }\end{array}$ & {$[63]$} \\
\hline & & $\mathrm{MeOH}$ followed by EtOH & [68] \\
\hline & & $\begin{array}{l}\text { Acidified }(0.01 \% \mathrm{HCl} 6 \mathrm{~N}) 40 \% \text { EtOH or } \\
\qquad \mathrm{H}_{2} \mathrm{O} \text { or } 70 \% \text { acetone }\end{array}$ & {$[72]$} \\
\hline
\end{tabular}


Table 1. Cont.

\begin{tabular}{|c|c|c|c|}
\hline Compound & Sample & Extraction Solvent & Reference \\
\hline & Purple corn kernel & $\mathrm{EtOH}-\mathrm{H}_{2} \mathrm{O}, 30: 70, v / v$ & [79] \\
\hline & & $\mathrm{H}_{2} \mathrm{O}$ or $\mathrm{H}_{2} \mathrm{O}+0.2 \% \mathrm{SO}_{2}$ or $\mathrm{H}_{2} \mathrm{O}+0.5 \%$ & \\
\hline & Purple corn pericarp & $\begin{array}{c}\text { lactic acid or } \mathrm{H}_{2} \mathrm{O}+0.2 \% \mathrm{SO}_{2}+0.5 \% \\
\text { lactic acid }\end{array}$ & [58] \\
\hline & & $\mathrm{H}_{2} \mathrm{O}$, Accelerated Solvent Extraction & {$[70,71,77,81,82]$} \\
\hline & Purple corn seeds & Acidified (1\% TFA) $\mathrm{MeOH}$ & [34] \\
\hline & Purple corn husk & $0.1 \mathrm{M} \mathrm{HCl}$ & [25] \\
\hline & Purple corn flour & $\mathrm{MeOH}$ acidified $1 \% \mathrm{HCl}$ & [18] \\
\hline & $\begin{array}{c}\text { Raw and germinated purple } \\
\text { corn }\end{array}$ & Acidified (1\% TFA) $80 \% \mathrm{MeOH}$ & [22] \\
\hline & Purple, red, blue corn pericarp & $70 \%$ aqueous acetone & [85] \\
\hline & & Acidified $(0.1 \mathrm{M} \mathrm{HCl}) 80 \% \mathrm{MeOH}$ & [50] \\
\hline & & $80 \% \operatorname{EtOH}(v / v)$, maceration & \\
\hline & & $80 \% \mathrm{EtOH}(v / v)$, ultra-sound assisted & \\
\hline & Corn cob and husk & extraction & [55] \\
\hline & & $\begin{array}{c}80 \% \operatorname{EtOH}(v / v), \text { microwave-assisted } \\
\text { extraction }\end{array}$ & \\
\hline \multirow{5}{*}{$\begin{array}{l}\text { Peonidin-3-O-(3"-6"'- } \\
\text { dimalonylglucoside) }\end{array}$} & Purple corn cob & Acidified $(0.01 \% \mathrm{HCl} 6 \mathrm{~N}) 70 \%$ acetone & [11] \\
\hline & Purple corn husk & $0.1 \mathrm{M} \mathrm{HCl}$ & [25] \\
\hline & Red purple corn & $2 \%$ aqueous formic acid & [37] \\
\hline & Purple, red corn kernel & $2 \%$ aqueous formic acid & {$[84]$} \\
\hline & Purple, red, blue corn pericarp & Acidified $(0.1 \mathrm{M} \mathrm{HCl}) 80 \% \mathrm{MeOH}$ & [50] \\
\hline Peonidin-3,5-diglucoside & Red purple corn & $2 \%$ aqueous formic acid & [37] \\
\hline Petunidin-3-O-glucoside & Purple corn cob & $\begin{array}{c}\mathrm{H}_{2} \mathrm{O} \text { or } \mathrm{EtOH} \text { or } \mathrm{H}_{2} \mathrm{O}: \mathrm{EtOH}, 1: 1, v / v \\
\text { ultrasonic assisted extraction }\end{array}$ & [63] \\
\hline $\begin{array}{l}\text { Malvidin-3-O-(6"- } \\
\text { dimalonylglucoside) }\end{array}$ & Purple corn & $\begin{array}{c}60-100 \% \text { EtOH, } w / w \text {, ultrasound } \\
\text { assisted extraction }\end{array}$ & {$[62]$} \\
\hline $\begin{array}{l}\text { Malvidin-3-O-(6"-malon- } p \text { - } \\
\text { hydroxybenzoilglucoside) }\end{array}$ & Purple corn & $\begin{array}{c}60-100 \% \text { EtOH, } w / w, \text { ultrasound } \\
\text { assisted extraction }\end{array}$ & {$[62]$} \\
\hline $\begin{array}{l}\text { Delphinidin-3-O-(6"- } \\
\text { malonylglucoside) }\end{array}$ & $\begin{array}{c}\text { Raw and germinated purple } \\
\text { corn }\end{array}$ & Acidified (1\% TFA) $80 \% \mathrm{MeOH}$ & [22] \\
\hline $\begin{array}{l}\text { Delphinidin-3-O-(6"-O-p- } \\
\text { coumaroylglucoside })\end{array}$ & Purple corn pericarp & $\mathrm{H}_{2} \mathrm{O}$, Accelerated Solvent Extraction & [77] \\
\hline $\begin{array}{l}\text { Catechin-(4,8)-cyanidin-3- } \\
\text { glucoside }\end{array}$ & Purple corn seeds & Acidified (1\% TFA) MeOH & {$[34]$} \\
\hline \multirow{5}{*}{$\begin{array}{c}\text { Catechin-(4,8)-cyanidin-3,5- } \\
\text { diglucoside }\end{array}$} & Purple corn cob & Acidified $(0.01 \% \mathrm{HCl} 6 \mathrm{~N}) 70 \%$ acetone & [11] \\
\hline & Purple corn pericarp & $\mathrm{H}_{2} \mathrm{O}$, Accelerated Solvent Extraction & {$[77,81]$} \\
\hline & Purple corn seeds & Acidified (1\% TFA) MeOH & {$[34]$} \\
\hline & $\begin{array}{l}\text { Raw and germinated purple } \\
\text { corn }\end{array}$ & Acidified (1\% TFA) $80 \% \mathrm{MeOH}$ & [22] \\
\hline & Red purple corn & $2 \%$ aqueous formic acid & [37] \\
\hline $\begin{array}{l}\text { Catechin-(4,8)-cyanidin-3- } \\
\text { malonylglucoside }\end{array}$ & Purple corn seeds & Acidified (1\% TFA) $\mathrm{MeOH}$ & [34] \\
\hline $\begin{array}{l}\text { Epicatechin- }(4,8) \text {-cyanidin-3- } \\
\text { malonylglucoside }\end{array}$ & Purple corn seeds & Acidified (1\% TFA) $\mathrm{MeOH}$ & {$[34]$} \\
\hline \multirow{4}{*}{$\begin{array}{l}\text { (Epi)catechin (4-8)-cyanidin } \\
\text { 3-malonylglucoside-5 } \\
\text { glucoside }\end{array}$} & & & \\
\hline & Purple corn seeds & Acidified (1\% TFA) MeOH & {$[34]$} \\
\hline & $\begin{array}{l}\text { Raw and germinated purple } \\
\text { corn }\end{array}$ & Acidified (1\% TFA) $80 \% \mathrm{MeOH}$ & [22] \\
\hline & Red purple corn & $2 \%$ aqueous formic acid & [37] \\
\hline
\end{tabular}


Table 1. Cont

\begin{tabular}{|c|c|c|c|}
\hline Compound & Sample & Extraction Solvent & Reference \\
\hline $\begin{array}{l}\text { Afzelechin-(4-8)-cyanidin 3,5 } \\
\text { diglucoside }\end{array}$ & Red purple corn & $2 \%$ aqueous formic acid & {$[37]$} \\
\hline $\begin{array}{l}\text { Catechin-(4,8)-pelargonidin-3- } \\
\text { glucoside }\end{array}$ & Purple corn seeds & Acidified (1\% TFA) MeOH & [34] \\
\hline \multirow[t]{2}{*}{$\begin{array}{l}\text { (Epi)catechin } \\
\text { (4-8)-pelargonidin 3,5 } \\
\text { diglucoside }\end{array}$} & $\begin{array}{l}\text { Raw and germinated purple } \\
\text { corn }\end{array}$ & Acidified (1\% TFA) $80 \% \mathrm{MeOH}$ & [22] \\
\hline & Red purple corn & $2 \%$ aqueous formic acid & [37] \\
\hline $\begin{array}{l}\text { Catechin (4-8)-pelargonidin } \\
\text { 3-malonylglucoside-5 } \\
\text { glucoside }\end{array}$ & Red purple corn & $2 \%$ aqueous formic acid & [37] \\
\hline $\begin{array}{l}\text { Afzelechin-(4-8)-pelargonidin } \\
\text { 3,5 diglucoside } \\
\text { Afzelechin- (4-8)-pelargonidin }\end{array}$ & Red purple corn & $2 \%$ aqueous formic acid & [37] \\
\hline $\begin{array}{l}\text { 3-malonylglucoside-5 } \\
\text { glucoside }\end{array}$ & Red purple corn & $2 \%$ aqueous formic acid & {$[37]$} \\
\hline $\begin{array}{l}\text { Catechin-(4,8)-peonidin-3- } \\
\text { glucoside }\end{array}$ & Purple corn seeds & Acidified (1\% TFA) MeOH & {$[34]$} \\
\hline $\begin{array}{l}\text { Epicatechin-(4,8)-peonidin-3- } \\
\text { glucoside }\end{array}$ & Purple corn seeds & Acidified (1\% TFA) MeOH & {$[34]$} \\
\hline $\begin{array}{l}\text { Catechin (4-8)-peonidin- 3,5 } \\
\text { diglucoside }\end{array}$ & Red purple corn & $2 \%$ aqueous formic acid & [37] \\
\hline $\begin{array}{l}\text { Catechin (4-8)-peonidin } \\
\text { 3-malonylglucoside-5 } \\
\text { glucoside }\end{array}$ & Red purple corn & $2 \%$ aqueous formic acid & [37] \\
\hline $\begin{array}{l}\text { Afzelechin-(4-8)-peonidin-3,5 } \\
\text { diglucoside }\end{array}$ & Red purple corn & $2 \%$ aqueous formic acid & [37] \\
\hline Proanthocyanidin dimer & Blue corn tortillas & Acidified (citric acid 1M) EtOH & [87] \\
\hline Proanthocyanidin trimer & Blue corn tortillas & Acidified (citric acid 1M) EtOH & [87] \\
\hline \multirow{2}{*}{$\begin{array}{l}\text { Proanthocyanidin tetramer } \\
\text { p-hydroxycinnamic acid }\end{array}$} & Blue corn tortillas & Acidified (citric acid 1M) EtOH & [87] \\
\hline & Purple corn kernel & $\begin{array}{c}\text { EtOH- } \mathrm{H}_{2} \mathrm{O}, 30: 70, v / v \\
35 \% \mathrm{EtOH}\end{array}$ & $\begin{array}{l}{[79]} \\
{[61]}\end{array}$ \\
\hline \multirow[t]{5}{*}{ Caffeic acid } & Purple corn cob & $30 \% \mathrm{EtOH}$ & [76] \\
\hline & Purple corn pericarp & $\mathrm{H}_{2} \mathrm{O}$, Accelerated Solvent Extraction & [82] \\
\hline & Purple waxy corn kernel & $80 \% \mathrm{EtOH}$ & [83] \\
\hline & Purple, red, blue corn flour & Acidified $(1 \% \mathrm{HCl}) \mathrm{MeOH}$ & [18] \\
\hline & Blue corn bean & Acidified $(0.1 \% \mathrm{HCl}) \mathrm{EtOH}$ & [86] \\
\hline Caffeic acid derivatives & Purple corn & $\begin{array}{l}\text { Acidified }(0.1 \% \mathrm{HCl}) \\
\text { MeOH:acetone: } \mathrm{H}_{2} \mathrm{O}, 45: 45: 10, v / v / v\end{array}$ & {$[90]$} \\
\hline Caffeic acid 4-O-hexoside & Red corn cob & $\begin{array}{c}55 \% \text { EtOH, ultrasonic-assisted } \\
\text { extraction } \\
\text { Acidified }(0.1 \% \mathrm{HCl})\end{array}$ & {$[54]$} \\
\hline \multirow[t]{9}{*}{ Ferulic acid } & Purple corn cob & $\begin{array}{c}\text { MeOH:acetone: } \mathrm{H}_{2} \mathrm{O}, 45: 45: 10, v / v / v+ \\
\mathrm{NaOH} 3 \mathrm{~N}+\text { ethyl acetate }\end{array}$ & {$[90]$} \\
\hline & & $\mathrm{MeOH}$ & [30] \\
\hline & & $30 \% \mathrm{EtOH}$ & [76] \\
\hline & Purple corn kernel & $\mathrm{EtOH}-\mathrm{H}_{2} \mathrm{O}, 30: 70, v / v$ & [79] \\
\hline & & $35 \% \mathrm{EtOH}$ & [61] \\
\hline & Purple corn pericarp & $\mathrm{H}_{2} \mathrm{O}$, Accelerated Solvent Extraction & [82] \\
\hline & Purple waxy corn kernel & $80 \% \mathrm{EtOH}$ & [83] \\
\hline & $\begin{array}{l}\text { Purple corn flour, orange } \\
\text { kernel }\end{array}$ & $80 \% \mathrm{MeOH}$ & {$[69,91]$} \\
\hline & Purple, red, blue corn flour & Acidified $(1 \% \mathrm{HCl}) \mathrm{MeOH}$ & [18] \\
\hline
\end{tabular}


Table 1. Cont

\begin{tabular}{|c|c|c|c|}
\hline Compound & Sample & Extraction Solvent & Reference \\
\hline \multirow{3}{*}{ Ferulic acid derivatives } & & Acidified $(0.1 \% \mathrm{HCl})$ & \\
\hline & Purple corn cob & $\begin{array}{c}\text { MeOH:acetone:H2O, 45:45:10, } v / v / v+ \\
\text { NaOH 3N + ethyl acetate }\end{array}$ & [90] \\
\hline & & Aqueous EtOH & [78] \\
\hline 3-O-feruloylquinic acid & Raw purple corn & Acidified (1\% TFA) $80 \% \mathrm{MeOH}$ & [22] \\
\hline 5-O-feruloylquinic acid & Purple corn cob & Aqueous EtOH & [78] \\
\hline Feruloyl-caffeoylglycerol & Purple, red, blue corn flour & Acidified $(1 \% \mathrm{HCl}) \mathrm{MeOH}$ & [18] \\
\hline $\mathrm{N}, \mathrm{N}^{\prime}$-diferuloylputrescine & Purple, red, blue corn flour & Acidified $(1 \% \mathrm{HCl}) \mathrm{MeOH}$ & [18] \\
\hline$o$-coumaric acid & Purple corn pericarp & $\mathrm{H}_{2} \mathrm{O}$, Accelerated Solvent Extraction & [26] \\
\hline \multirow[t]{6}{*}{$p$-coumaric acid } & Orange kernel & $80 \% \mathrm{MeOH}$ & [69] \\
\hline & Purple corn cob & $\begin{array}{l}\text { Acidified }(0.1 \% \mathrm{HCl}) \\
\text { MeOH:acetone: } \mathrm{H}_{2} \mathrm{O}, 45: 45: 10, v / v / v+ \\
\mathrm{NaOH} 3 \mathrm{~N}+\text { ethyl acetate }\end{array}$ & {$[90]$} \\
\hline & Purple corn cob & $30 \% \mathrm{EtOH}$ & [76] \\
\hline & Purple waxy corn kernel & $80 \% \mathrm{EtOH}$ & [83] \\
\hline & Red corn cob & $\begin{array}{c}55 \% \text { EtOH, ultrasonic-assisted } \\
\text { extraction }\end{array}$ & {$[54]$} \\
\hline & Blue corn bean & Acidified $(0.1 \% \mathrm{HCl}) \mathrm{EtOH}$ & [86] \\
\hline$p$-coumaroylquinic acid & Green, pinky, purple corn silk & $95 \% \mathrm{MeOH}$ & {$[27]$} \\
\hline$p$-coumaric acid derivatives & Purple corn & $\begin{array}{c}\text { Acidified }(0.1 \% \mathrm{HCl}) \\
\text { MeOH:acetone: } \mathrm{H}_{2} \mathrm{O}, 45: 45: 10, v / v / v\end{array}$ & {$[90]$} \\
\hline \multirow{2}{*}{$\begin{array}{c}\text { Coumaroyl-caffeoylglycerol } \\
\text { N-coumaroyl-N'- } \\
\text { feruloylputrescine }\end{array}$} & Purple, red, blue corn flour & Acidified $(1 \% \mathrm{HCl}) \mathrm{MeOH}$ & [18] \\
\hline & Purple, red, blue corn flour & Acidified $(1 \% \mathrm{HCl}) \mathrm{MeOH}$ & [18] \\
\hline $\mathrm{N}, \mathrm{N}^{\prime}$-dicoumaroylspermidine & Purple, red, blue corn flour & Acidified $(1 \% \mathrm{HCl}) \mathrm{MeOH}$ & [18] \\
\hline \multirow[t]{6}{*}{ Protocatechuic acid } & Purple corn cob & $30 \% \mathrm{EtOH}$ & [76] \\
\hline & Purple corn flour & $80 \% \mathrm{MeOH}$ & [91] \\
\hline & Purple corn kernel & $\mathrm{EtOH}-\mathrm{H}_{2} \mathrm{O}, 30: 70, v / v$ & [79] \\
\hline & & $35 \% \mathrm{EtOH}$ & {$[61]$} \\
\hline & Purple corn pericarp & $\mathrm{H}_{2} \mathrm{O}$, Accelerated Solvent Extraction & [82] \\
\hline & Purple waxy corn kernel & $80 \% \mathrm{EtOH}$ & [83] \\
\hline Sinapic acid & Purple corn cob & $30 \% \mathrm{EtOH}$ & [76] \\
\hline Syringic acid & Purple corn cob & $30 \% \mathrm{EtOH}$ & [76] \\
\hline & & $\mathrm{MeOH}$ & [30] \\
\hline \multirow[t]{2}{*}{ 3-O-caffeoylquinic acid } & $\begin{array}{l}\text { Raw and germinated purple } \\
\text { corn }\end{array}$ & Acidified (1\% TFA) $80 \% \mathrm{MeOH}$ & [22] \\
\hline & Green, pinky, purple corn silk & $95 \% \mathrm{MeOH}$ & [29] \\
\hline 4-O-caffeoylquinic acid & Green, pinky, purple corn silk & $95 \% \mathrm{MeOH}$ & [29] \\
\hline 5-O-caffeoylquinic acid & Green, pinky, purple corn silk & $95 \% \mathrm{MeOH}$ & [29] \\
\hline \multirow[b]{3}{*}{$\begin{array}{l}\text { Dicaffeoyl-succinoylquinic } \\
\text { acid }\end{array}$} & Red corn cob & $\begin{array}{l}55 \% \mathrm{EtOH} \text {, ultrasonic-assisted } \\
\text { extraction }\end{array}$ & {$[54]$} \\
\hline & Blue corn bean & Acidified $(0.1 \% \mathrm{HCl}) \mathrm{EtOH}$ & [86] \\
\hline & $\begin{array}{c}\text { Raw and germinated purple } \\
\text { corn }\end{array}$ & Acidified (1\% TFA) $80 \% \mathrm{MeOH}$ & [22] \\
\hline \multirow[t]{4}{*}{ p-Hydroxybenzoic acid } & Orange kernel & $80 \% \mathrm{MeOH}$ & [32] \\
\hline & Raw purple corn & Acidified (1\% TFA) $80 \% \mathrm{MeOH}$ & [22] \\
\hline & Purple corn cob & $\mathrm{MeOH}$ & {$[30]$} \\
\hline & & $30 \% \mathrm{EtOH}$ & [76] \\
\hline \multirow{8}{*}{ Vanillic acid } & Purple waxy corn kernel & $80 \% \mathrm{EtOH}$ & [83] \\
\hline & Purple corn cob & $\mathrm{MeOH}$ & [30] \\
\hline & & $30 \% \mathrm{EtOH}$ & [76] \\
\hline & Purple corn kernel & $\mathrm{EtOH}-\mathrm{H}_{2} \mathrm{O}, 30: 70, v / v$ & [79] \\
\hline & & $35 \% \mathrm{EtOH}$ & [61] \\
\hline & Purple corn pericarp & $\mathrm{H}_{2} \mathrm{O}$, Accelerated Solvent Extraction & [82] \\
\hline & $\begin{array}{l}\text { Raw and germinated purple } \\
\text { corn }\end{array}$ & Acidified (1\% TFA) $80 \% \mathrm{MeOH}$ & {$[22]$} \\
\hline & Purple waxy corn kernel & $80 \% \mathrm{EtOH}$ & [83] \\
\hline
\end{tabular}


Table 1. Cont.

\begin{tabular}{|c|c|c|c|}
\hline Compound & Sample & Extraction Solvent & Reference \\
\hline Vanillic acid hexoside & Germinated purple corn & Acidified (1\% TFA) $80 \% \mathrm{MeOH}$ & [22] \\
\hline \multirow[t]{2}{*}{ 2,4,6-tribenzoic acid } & \multirow[t]{2}{*}{ Purple corn kernel } & $\mathrm{EtOH}-\mathrm{H}_{2} \mathrm{O}, 30: 70, v / v$ & [79] \\
\hline & & $35 \% \mathrm{EtOH}$ & [61] \\
\hline Kaempferol & Purple corn pericarp & $\mathrm{H}_{2} \mathrm{O}$, Accelerated Solvent Extraction & [82] \\
\hline Kaempferol O-hexoside & Germinated purple corn & Acidified (1\% TFA) 80\% MeOH & [22] \\
\hline Kaempferol-3-O-glucoside & Raw purple corn & Acidified (1\% TFA) $80 \% \mathrm{MeOH}$ & [22] \\
\hline Kaempferol-7-O-glucoside & \multirow{2}{*}{$\begin{array}{c}\text { Purple corn cob } \\
\text { Raw and germinated purple } \\
\text { corn }\end{array}$} & Aqueous EtOH & [78] \\
\hline Kaempferol acetylhexoside & & Acidified (1\% TFA) $80 \% \mathrm{MeOH}$ & [22] \\
\hline \multirow{2}{*}{$\begin{array}{c}\text { Keampfero-3,7-di-O-hexoside } \\
\text { Kaempferol rhamnoside } \\
\text { hexoside }\end{array}$} & \multirow{2}{*}{$\begin{array}{c}\text { Purple corn cob } \\
\text { Raw and germinated purple } \\
\text { corn }\end{array}$} & Aqueous EtOH & [78] \\
\hline & & Acidified (1\% TFA) $80 \% \mathrm{MeOH}$ & [22] \\
\hline $\begin{array}{c}\text { Keampferolo-7-O-(6"- } \\
\text { malonylglucoside) }\end{array}$ & Purple corn cob & Aqueous EtOH & [78] \\
\hline \multirow{4}{*}{$\begin{array}{l}\text { Kaempferol-7-O-rutinoside } \\
\text { kaempferol-3-O-hexosyl-7-O- } \\
\text { glucuronilhexoside } \\
\text { Quercetin }\end{array}$} & Purple corn cob & Aqueous EtOH & [78] \\
\hline & Purple corn cob & Aqueous $\mathrm{EtOH}$ & [78] \\
\hline & Purple corn cob & $30 \% \mathrm{EtOH}$ & [76] \\
\hline & Purple corn pericarp & $\mathrm{H}_{2} \mathrm{O}$, Accelerated Solvent Extraction & [82] \\
\hline Quercetin-O-glucoside & $\begin{array}{c}\text { Raw and germinated purple } \\
\text { corn }\end{array}$ & Acidified (1\% TFA) 80\% MeOH & {$[22]$} \\
\hline Quecertin-7-O-glucoside & Purple corn cob & Aqueous EtOH & [78] \\
\hline Quercetin 3-O-hexoside & $\begin{array}{l}\text { Raw and germinated purple } \\
\text { corn }\end{array}$ & Acidified (1\% TFA) $80 \% \mathrm{MeOH}$ & [22] \\
\hline \multirow[t]{3}{*}{ Quercetin-3-O-rutinoside } & Purple corn cob & $30 \% \mathrm{EtOH}$ & {$[76]$} \\
\hline & Purple corn pericarp & $\mathrm{H}_{2} \mathrm{O}$, Accelerated Solvent Extraction & {$[77,82]$} \\
\hline & $\begin{array}{l}\text { Raw and germinated purple } \\
\text { corn }\end{array}$ & Acidified (1\% TFA) $80 \% \mathrm{MeOH}$ & [22] \\
\hline $\begin{array}{l}\text { Quecertin-7-O-p- } \\
\text { coumaroylhexoside }\end{array}$ & Purple corn cob & Aqueous EtOH & [78] \\
\hline Myricetin-7-O-hexoside & Purple corn cob & Aqueous $\mathrm{EtOH}$ & [78] \\
\hline Myricetin-3,7-di-O-glucoside & Purple corn cob & Aqueous $\mathrm{EtOH}$ & [78] \\
\hline \multirow[t]{2}{*}{ Isorhamnetin-O-hexoside } & Raw purple corn & Acidified (1\% TFA) $80 \% \mathrm{MeOH}$ & [22] \\
\hline & Purple corn cob & Aqueous EtOH & [78] \\
\hline Isorhamnetin-3-O-glucoside & $\begin{array}{l}\text { Raw and germinated purple } \\
\text { corn }\end{array}$ & Acidified (1\% TFA) $80 \% \mathrm{MeOH}$ & [22] \\
\hline Isorhamnetin-3-O-rutinoside & $\begin{array}{l}\text { Raw and germinated purple } \\
\text { corn }\end{array}$ & Acidified (1\% TFA) $80 \% \mathrm{MeOH}$ & [22] \\
\hline \multirow{2}{*}{$\begin{array}{c}\text { Isorhamnetin-7-O-rutinoside } \\
\text { Isorhamnetin-3,7-di-O- } \\
\text { hexiside }\end{array}$} & Purple corn cob & Aqueous EtOH & [78] \\
\hline & Purple corn cob & Aqueous EtOH & {$[78]$} \\
\hline $\begin{array}{l}\text { Isorhamentin-3-O-hexosyl-7- } \\
\text { O-glucuronilhexoside }\end{array}$ & Purple corn cob & Aqueous EtOH & [78] \\
\hline \multirow[t]{2}{*}{ Apigenin-O-hexoside } & Red corn cob & $\begin{array}{c}55 \% \text { EtOH, ultrasonic-assisted } \\
\text { extraction }\end{array}$ & {$[54]$} \\
\hline & Blue corn bean & Acidified $(0.1 \% \mathrm{HCl}) \mathrm{EtOH}$ & [86] \\
\hline \multirow{6}{*}{$\begin{array}{c}\text { Apigenin-7-O-glucoside } \\
\text { Apigenin-O-pentosyl } \\
\text { hexoside } \\
\text { di-C,C-hexosyl-apigenin } \\
\text { C-hexosyl apigenin } \\
\text { C-hexosyl-C-pentosyl } \\
\text { apigenin }\end{array}$} & Purple corn cob & Aqueous EtOH & [78] \\
\hline & Red corn cob & $\begin{array}{c}55 \% \text { EtOH, ultrasonic-assisted } \\
\text { extraction }\end{array}$ & {$[54]$} \\
\hline & Red purple corn & $2 \%$ aqueous formic acid & [37] \\
\hline & Red purple corn & $2 \%$ aqueous formic acid & {$[37]$} \\
\hline & Red purple corn & $2 \%$ aqueous formic acid & [37] \\
\hline & Red corn cob & $\begin{array}{c}55 \% \text { EtOH, ultrasonic-assisted } \\
\text { extraction }\end{array}$ & [54] \\
\hline
\end{tabular}


Table 1. Cont.

\begin{tabular}{|c|c|c|c|}
\hline Compound & Sample & Extraction Solvent & Reference \\
\hline Luteolin & Purple corn pericarp & $\mathrm{H}_{2} \mathrm{O}$, Accelerated Solvent Extraction & [82] \\
\hline Luteolin-7-O-glucoside & Purple corn cob & \multirow{2}{*}{$\begin{array}{c}\text { Aqueous EtOH } \\
\text { 55\% EtOH, ultrasonic-assisted } \\
\text { extraction }\end{array}$} & [78] \\
\hline Luteolin-O-rutinoside & Red corn cob & & {$[54]$} \\
\hline $\begin{array}{l}\text { 2"-O-pentoside-8-C-hexoside } \\
\text { Luteolin }\end{array}$ & $\begin{array}{c}\text { Raw and germinated purple } \\
\text { corn }\end{array}$ & Acidified (1\% TFA) 80\% MeOH & [22] \\
\hline Naringenin & Purple corn pericarp & $\mathrm{H}_{2} \mathrm{O}$, Accelerated Solvent Extraction & {$[77,82]$} \\
\hline \multirow[t]{2}{*}{ Daidzin } & Red corn cob & $\begin{array}{c}55 \% \mathrm{EtOH}, \text { ultrasonic-assisted } \\
\text { extraction }\end{array}$ & [54] \\
\hline & Blue corn bean & \multirow{2}{*}{$\begin{array}{c}\text { Acidified }(0.1 \% \mathrm{HCl}) \mathrm{EtOH} \\
55 \% \text { EtOH, ultrasonic-assisted } \\
\text { extraction }\end{array}$} & [86] \\
\hline Procyanidin dimer & Red corn cob & & {$[54]$} \\
\hline Scopoletin & Red corn cob & $\begin{array}{c}55 \% \text { EtOH, ultrasonic-assisted } \\
\text { extraction }\end{array}$ & {$[54]$} \\
\hline Maysin & Green, pinky, purple corn silk & $95 \% \mathrm{MeOH}$ & [25] \\
\hline Maysin derivative & Green, pinky, purple corn silk & $95 \% \mathrm{MeOH}$ & [25] \\
\hline Methoxymaysin derivative & Green, pinky, purple corn silk & $95 \% \mathrm{MeOH}$ & [25] \\
\hline Vitexin & Red purple corn & $2 \%$ aqueous formic acid & [37] \\
\hline Isovitexin & Red purple corn & $2 \%$ aqueous formic acid & {$[37]$} \\
\hline \multirow[t]{2}{*}{ Hirsutrin } & \multirow[t]{2}{*}{ Purple corn kernel } & $\mathrm{EtOH}-\mathrm{H}_{2} \mathrm{O}, 30: 70, v / v$ & [79] \\
\hline & & $35 \% \mathrm{EtOH}$ & {$[61]$} \\
\hline \multirow[t]{2}{*}{$3^{\prime}$-methoxyhirsutrin } & \multirow[t]{2}{*}{ Purple corn kernel } & $\mathrm{EtOH}-\mathrm{H}_{2} \mathrm{O}, 30: 70, v / v$ & [79] \\
\hline & & $35 \% \mathrm{EtOH}$ & {$[61]$} \\
\hline GABA & Germinated purple corn & $\begin{array}{c}\mathrm{H}_{2} \mathrm{O}+\text { derivatization by phenyl } \\
\text { isothiocyanate }\end{array}$ & [22] \\
\hline \multirow[t]{2}{*}{ Lutein } & Orange kernel & $\mathrm{H}_{2} \mathrm{O}$-saturated butan-1-ol & {$[69,92]$} \\
\hline & Red kernel & $\begin{array}{c}\text { Diethyl ether:petroleum } \\
\text { ether, } 2: 1, v / v\end{array}$ & [47] \\
\hline \multirow[t]{2}{*}{ Zeaxanthin } & Orange kernel & $\mathrm{H}_{2} \mathrm{O}$-saturated butan-1-ol & {$[69,92]$} \\
\hline & Red kernel & $\begin{array}{c}\text { Diethyl ether:petroleum } \\
\text { ether, } 2: 1, v / v\end{array}$ & {$[47]$} \\
\hline \multirow[t]{2}{*}{$\beta$-Cryptoxanthin } & Orange kernel & Water-saturated butan-1-ol & {$[69,92]$} \\
\hline & Red kernel & $\begin{array}{c}\text { Diethyl ether:petroleum } \\
\text { ether, } 2: 1, v / v\end{array}$ & {$[47]$} \\
\hline \multirow[t]{2}{*}{$\beta$-Carotene } & Orange kernel & $\mathrm{H}_{2} \mathrm{O}$-saturated butan-1-ol & {$[69,92]$} \\
\hline & Red kernel & $\begin{array}{c}\text { Diethyl ether:petroleum } \\
\text { ether, } 2: 1, v / v\end{array}$ & [47] \\
\hline
\end{tabular}

\section{Health Benefits}

Several in vitro and in vivo studies correlated the healthy properties, i.e., antibacterial, antimutagenic, antioxidant, anti-inflammatory, anticarcinogenic, hypoglycemic, and hypolipidemic, of pigmented corn varieties mainly to their carotenoid, anthocyanin, and polyphenolic content. The different qualitative and quantitative profile can justify the different activity reported in literature, as discussed below.

\subsection{Antibacterial and Antimutagenic Activities}

Polyphenols are known to be antimicrobial agents and this activity against pathogenic bacteria is related to the chemical structure and concentration of each compound and it can also vary dependently on the fact that each molecule is tested alone or in a mixture, and therefore, the composition of the extract can highly affect the activity. Both free and bound phenolic fractions extracted from powdered Peruvian purple corn accession (AREQ-084) were tested on the growth of two probiotic lactic acid bacteria strains, i.e., Lactobacillus helveticus and Bifidobacterium longum, and of Helicobacter pylori, the main responsible pathogen for gastric cancer development [79]. Free phenolic fraction was mainly characterized by the presence of anthocyanins (cyanidin-, peonidin-, pelargonidin-3-O-glucoside, and 
their malonyl derivatives); conversely, bound phenolic fraction was mainly composed of $p$-coumaric acid, ferulic acid, and its derivatives. No effect on the beneficial growth of probiotic bacteria was registered for free phenolic fraction tested in the range $10-50 \mathrm{mg} / \mathrm{mL}$ of medium (corresponding to a concentration range of $0.03-0.15 \mathrm{mg} \mathrm{C} 3 \mathrm{G}$ equivalents $/ \mathrm{mL}$ medium) and for bound phenolic fraction tested at $10 \mathrm{mg} / \mathrm{mL}$ of medium (corresponding to 0.02 and $0.016 \mathrm{mg}$ phenolic acids and ferulic acid $/ \mathrm{mL}$ medium, respectively). The same concentrations were tested against the growth of $H$. pylori and no inhibitory activity was registered, differently from the results published for anthocyanins and anthocyanin-rich extracts from cranberry against other Gram-negative bacteria [93]. However, these data were in accordance with previously reported results indicating a similar range of anthocyanin from another source not active on $H$. pylori amount, but active on its virulence factors $[94,95]$. No relationship between total polyphenol content and antibacterial activity against Staphylococcus aureus, Bacillus subtilis, Escherichia coli, and Pseudomonas aeruginosa was registered for different germplasms of corn (white, yellow, and purple) from two different Costa Rica regions, even if a purple variety had high activity against $B$. subtilis, indicating that other secondary metabolites can highly contribute to the biocidal action [96].

The antimutagenic activity of eleven genotypes of Mexican pigmented corn (red and blue) was evaluated testing the corresponding flavonoid and anthocyanin extracts using TA100 and TA98 Salmonella typhimurium strains and aflatoxin B1 (AFB1) mutagen. No extract was toxic with respect to the bacteria, but flavonoid extracts, rich in ferulic acid, were able to inhibit from $43 \%$ to $93 \%$ AFB1 mutagenicity with higher capacity to inhibit frameshift mutation in the genoma with respect to base changes mutations. The same trend was registered for the anthocyanin fraction, even if with lower percentage values. In particular blue corn samples, rich in C3G, showed higher efficacy against AFB1 on TA98 than TA100, differently from previously demonstrated for another blue Mexican variety [38,97], but these differences could be attributed to the different methods used to test the antimutagenic activity and also to the different composition in anthocyanins. Furthermore, in the case of flavonoid extracts, the different capacity registered for the tested samples could depend on the different extract composition: in fact, steric and electronic properties of each flavonoid differently affect the capacity to bind bacteria membrane and, thus, penetrate into them [38].

\subsection{Effects on Metabolic Syndrome, Diabetes, Glucose and Lipidic Metabolism}

Among the various biological activities reported for polyphenol, and in particular for anthocyanins, the action of the different corn extracts on metabolic syndrome had recently attracted the attention of the researchers. Today, obesity and diabetes are increasing day by day and World Health Organization estimated about $40 \%$ of adults and 38 million children are overweight and 1.6 million deaths were estimated to be caused by type- 2 diabetes (T2D) in 2016 [98,99]. The search for new antioxidant molecules able to maintain redox status and reduce insulin resistance and inflammation determined by over expression of adipokines, which are signaling factors involved in the regulation of blood pressure, energy and vascular homeostasis, and inflammation, is mandatory. In the last decades, several published research pointed out the anti-diabetic, anti-obesity, and anti-inflammatory potential properties of anthocyanin-based extracts by using in vitro and in vivo model tests and many mechanisms of action have been proposed. Generally, biological models, i.e., cell cultures, offer approaches to study the phytochemicals activity which better correlate with their action in vivo.

Free and bound phenolic fraction obtained by different Peruvian corn accessions were tested in vitro for their capacity to inhibit target enzymes for hyperglycemia and obesity, i.e., $\alpha$-glucosidase, $\alpha$-amylase, and lipase activities. Free phenolic fractions generally had a higher dose-dependent activity and darker purple samples highly inhibited lipase and $\alpha$-glucosidase, but only a mild activity against $\alpha$-amylase was registered for the same samples, thus leading to promising agents, able to modulate the glycemic levels without abnormal bacterial fermentation of undigested starch in the gut produced by a weak $\alpha$ - 
amylase activity. A correlation between the anthocyanin content and both $\alpha$-glucosidase and lipase inhibitory activity was highlighted suggesting a high contribution of these compounds to the registered antihyperglycemic and anti-obesity effects [100]. Similar results were also obtained for a cob extract obtained by a Lombard purple corn variety, namely Moradyn [78].

As purified compounds may exert different biological activities in relation to their chemical structure and the presence of more than one molecule in extracts can exert synergistic or antagonist action, Luna-Vital et al. [80] compared the effect of a purple corn pericarp extract and pure anthocyanins on inflammation, adipogenesis, and insulinresistance using 3T3-L1 cells as in vitro model and performing the assays both in basal and inflammatory conditions. The extract inhibited the adipocytes differentiation by reducing peroxisome proliferator-activated receptors (PPAR) $\gamma$-expression in a dose dependent manner (IC50 $0.4 \mathrm{mg} / \mathrm{mL}$ and $38.4 \mu \mathrm{g} / \mathrm{mL}$, respectively) and promoted the reduction of lipid concentration especially in basal conditions, differently from the pure single anthocyanins; in fact, a lower triglycerides concentration was detected in presence of IC50 extract, but the same concentration promoted the increase in triglyceride concentration when a postprandial status was mimicked in the assay evaluating the glucose-dependent lipolysis in 3T3-L1 cells. In addition, the extract reduced the lipogenic and lipolytic enzymes in a dose dependent manner through an interaction with amino acid residues of the catalytic cavity of lipases and different domains of fatty acids synthase, but with IC50 value higher than that of the isolated proanthocyanidin fraction which in turn was higher than those of the isolated anthocyanins. Finally, the extract exerted a general positive effect on insulin-resistant adipocytes in which inflammation was promoted by tumor necrosis factor (TNF)- $\alpha$, and induced a decrease of leptin and an increase of adiponectin. Intracellular reactive oxygen species (ROS) levels were reduced to basal level both when adipocytes were pre-treated with TNF- $\alpha$ and after treated with extract and when simultaneously treated with TNF- $\alpha$ and extract, differently from pure anthocyanins which generally were more active when the simultaneous approach was used. An improvement of insulin sensitivity was also obtained through glucose transporter type 4 (GLUT4) membrane translocation and the modulation of the phosphorylation pattern of insulin pathway. The same extract and its purified compounds were also active on other two T2D targets, i.e., free fatty acid receptor-1 (FFAR-1) and glucokinase (GK) responsible for the stimulation of pancreatic $\beta$-cells to secrete glucose-dependent insulin and the preservation of glucose homeostasis at pancreatic and liver level, respectively. In general, the extract exerted a better activation of FFAR-1 and GK than the pure compounds with IC50 value of $77 \mu \mathrm{g} / \mathrm{mL}$ in INS-1E dual-layer cell culture and of $44 \mu \mathrm{g} / \mathrm{mL}$ in HepG2 cells; therefore, the anthocyanin fraction could be considered a better candidate for ameliorating T2D treatment than pure compounds [81].

The anthocyanin fraction of 20 different purple corn lines deriving from the landrace Apache Red, each with a characteristic polyphenolic profile, was tested using murine lipopolysaccharide (LPS)-activated RAW264.7 macrophages and TNF- $\alpha$-induced 3T3-L1 adipocytes for testing the anti-diabetic, anti-adipogenic, and anti-inflammatory capacities. The results indicated differences among the genotypes, but generally all samples reduced the production of some target parameters associated to obesity such as the main proinflammatory mediators in adipose tissue, i.e., TNF- $\alpha$, prostaglandin E2, interleukin- 6 (IL6), nitric oxide (NO), LPS, monocyte chemoattractant protein-1 (generally present in high level in obese subjects), leptin, and enhanced the level of adiponectin (an anti-inflammatory mediator). Moreover, the extracts lowered the lipid levels by a cell proliferation inhibition or by a decrease of preadipocyte-adipocyte transition. In this last case, the mechanism of action involved the inhibition of the expression level of PPAR, and it was demonstrated only for some samples as well as the reduction of the expression level of the enzyme fatty acid synthase involved in lipogenesis and therefore indirectly in the control of postprandial blood glucose alteration. Colored pericarp extracts were also able to inhibit $\alpha$-amylase and a serine aminopeptidase inactivating the glucagon-like peptide- 1 with a stronger action than other sources rich in polyphenols [101,102], showing IC50 values ranging from 109.5 
to $172.7 \mu \mathrm{g} / \mathrm{mL}$ and from 65.5 to $702.7 \mu \mathrm{g} / \mathrm{mL}$, respectively. The hypoglycemic effects were also supported by the ability to reduce about 30-60\% ROS levels and to restore the glucose uptake (about 30-140\%) in TNF- $\alpha$-induced 3T3-L1 adipocytes. These results supported the potential use of colored corn pigments as functional ingredients for the reduction of the risk of T2D and are fortified by the anti-inflammatory and anti-diabetic actions of quercetin, luteolin, and rutin, and by the anti-adipogenic potential of vanillic and protocatechuic acids generally present in the colored extracts [82].

Another important promising target protein for T2D and obesity treatment is tyrosine phosphatase $1 \beta$ (PTP1 $\beta$ ) which is expressed in different insulin-sensitive tissue. Therefore, some compounds isolated from commercially purple corn kernel were tested using a suitable in vitro kit based on the co-incubation of samples with the substrate $\mathrm{N} p$-nitrophenyl phosphate (pNPP). The isolated fraction containing phenolic acids and flavonoids had a moderate PTP1 $\beta$ inhibitory effect (IC50 $26.12 \mu \mathrm{g} / \mathrm{mL}$ ), even if higher than that of the anthocyanin fraction (IC50 $58.20 \mu \mathrm{g} / \mathrm{mL}$ ). The most active compounds were ferulic acid, 3-methoxyhirsutrin, pelargonidin-3-O-glucoside, and cyanidin-3-O-(malonylglucoside); this last one acted with a noncompetitive inhibition of PTP1 $\beta$ [61].

Recently, the antiobesity activity on adipocyte life cycle of a purple corn silk extract was investigated. Chlorogenic acid, quercetin, and naringenin derivatives were indicated as the main responsible for the capacity to reduce significantly the pre-adipocyte proliferation; conversely, the anti-differentiation properties by decreasing the lipid accumulation evidenced for the extract at 0.5 and $1 \mathrm{mg} / \mathrm{mL}$ were mainly attributed to quercetin, anthocyanins, and vanillic acid which acted by down-regulating enzymes involved in the adipogenesis and PPAR $\gamma$ expression. Moreover, a moderate lipolysis stimulating effect, which could be a promising therapeutic strategy in the treatment of obesity, was pointed out as demonstrated by the increasing of released glycerol contents in adipocytes, in addition to a moderate apoptosis induction. The overall results obtained in this research indicated purple corn silk as a promising by-product useful in the prevention of obesity, even if in vivo studies are mandatory [26].

The promising results obtained by the above mentioned in vitro studies were generally confirmed by in vivo studies; in fact, purple corn kernel extract provided by the Corn Research Institute in Gangwon Province, Korea, differently affected the metabolic parameters in T2D db/db mice treated for 8 week with 10 or $50 \mathrm{mg}$ dry material $/ \mathrm{kg}$ : In fact, it significantly decreased blood glucose (52\% and $68 \%$, respectively), Hemoglobin A1c (HbA1c) (about 20\%), and glucagone (40\%) blood levels, but increased the serum insulin (at least of 1.5 fold), C-peptide (at least of 1.4 fold), and adiponectin levels compared to diabetic mice control group. Lower triglyceride levels and higher High-Density Lipoprotein (HDL)-cholesterol levels were also registered in treated groups. The extract determined a suppression of blood glucose level after 30-90 min from glucose load in the oral glucose tolerance test and prevented islet destruction as demonstrated by histological examination. Finally, it activated 5'-adenosine monophosphate-activated protein kinase (AMPK), thus reducing the hepatic glucose production and over expressing mRNA of GLUT4, thus increasing the flux of glucose into skeletal muscle. These results confirmed that purple corn extract is a good candidate for hyperglycemia and hyperlipidemia control in T2D [79].

The effect on metabolic syndrome was also demonstrated in vivo, as shown in the study of Bhaswant et al. [103]. The administration to mail Wistar rats of a high-carbohydrate or high-fat diets, corresponding to $1.8 \mathrm{~g} /$ day (9.1 mg anthocyanins $/ \mathrm{kg} /$ day) or $1.5 \mathrm{~g} / \mathrm{day}$ of purple corn flour (7.4 mg anthocyanins $/ \mathrm{kg} /$ day) showed an important impact on metabolic syndrome; in fact, glucose tolerance improved, systolic blood pressure, total body fat mass, and visceral adiposity index were reduced. In animals fed with a high-fat diet, alkaline phosphatase, alanine transaminase, and aspartate transaminase were reduced, even if these parameters did not be normalized, and plasma triacylglycerols and total cholesterol levels significantly decreased. A lower anthocyanins concentration $(2.92 \mathrm{mg} / \mathrm{kg})$ from blue corn extract was added both to high-sucrose and high-cholesterol-high-sucrose diets of 
Wistar rats. The obtained results were again promising as higher high-density lipoprotein cholesterol and lower systolic blood pressure, total cholesterol, serum triglycerides, and epididymal adipose tissue weight were registered in the treated animal group in respect to the control [104].

Purple corn anthocyanins also positively affected the lipid metabolism in lactating rats supplemented with chia oil rich in $\alpha$-linolenic acid (ALA), in particular they could decrease gene expression of Sterol Regulatory Element-Binding transcription factor 1 (SREBP-1c) in the liver, differently from ALA which would increase the expression of PPAR- $\alpha$. These actions alter hepatic $\Delta 5$ desaturase gene expression only apparently because the purple corn extract protect desaturases activity, which is reduced by high level of ALA with a mechanism not yet totally elucidated [105].

In 2020, the potential mechanisms of interaction of blue corn anthocyanins with target cell signaling proteins involved in inflammation, insulin resistance, oxidative stress, glucose and lipid metabolism, and therefore, in the development of diabetes, was predicted using in silico approach. 312 different interactions between compounds and proteins were detected and the main important occurred between C3G and 11 $\beta$-hydroxysteroid dehydrogenase (-HSD), glutamine fructose-6-phosphate amidotransferase (GFAT), PPAR $\gamma$, or petunidin-3O-glucoside, $11 \beta$-HS and protein tyrosine phosphatase (PTP) or delphinidin 3-O-glucoside, and $11 \beta-H S D$, GFAT, PTP, and receptor tyrosine kinases. These molecular docking results gave an important contribution to the search for new molecules with anti-diabetic effect and thanks to these predictions new pathways by which anthocyanins exert their effects can be studied [86].

Diabetic neuropathy is one of the most common complication of diabetes, which can be caused by an increase in oxidative stress status, in advanced end glycation products formation, and aldose reductase activity. A 50\% hydroalcoholic extract constituted of Zingiber officinale rhizomes and purple Zea mays seeds administered to streptozotocindiabetic rats improved neuropathy with a mechanism not related to the reduction of blood glucose. Moreover, this extract reduced myelin degradation, thus increasing myelinated nerve fiber density, which is crucial for maintaining postural stability in rats subjected to chronic constriction at sciatic nerve, and decreased neuropathic pain by a reduction in the stimulation of pain fiber as a consequence of a reduced oxidative stress [106].

\subsection{Anti-Inflammatory Properties}

Cyclooxygenase-2 (COX-2) and inducible NO synthase (iNOS) are the key factors in the inflammation process and their inhibition could be beneficial. Proanthocyanidin (mainly with degree of polymerization $<10$ ) fraction of purple and red corn pericarp had the capacity to inhibit over $89 \%$ COX-2 when tested at the concentration of $1 \mathrm{mg} / \mathrm{mL}$ without any effect on COX-1, the key enzyme in homeostatic regulation. The same compounds and C3G present in purple corn were also dose-dependent $(0.1-1 \mathrm{mg} / \mathrm{mL})$ non-competitive inhibitors of iNOS [85]. As recently reported by Zhang et al. [107], anthocyanins present in purple and red corn also ameliorated the paracrine interplay between adipocytes and macrophages, reducing the production of TNF- $\alpha$ and Monocyte Chemoattractant Protein-1 (MCP-1) in both macrophages and adipocytes mono-cultures, and inhibiting nuclear factor kappa-light-chain-enhancer of activated B cells (NF-kB) and c-Jun N-terminal Kinase (JNK) pathways activation.

\subsection{Effect on Oxidative Stress}

An imbalance between the generation of free radicals (ROS and reactive nitrogen species (RNS)) and the antioxidant defense systems caused oxidative stress. Both enzymatic and non-enzymatic systems can contribute to the defense of cell structures and many different molecules other than micronutrients and minerals are known to prevent radical formation or to scavenge the produced radicals. Oxidative stress and inflammation are associated to many diseases, and also to obesity, a complex disorder co-responsible of the increased morbidity and mortality in the last decades. A purple corn extract contain- 
ing 52.02\% C3G and its malonyl derivative (27.33\%), 13.58\% peonidin-3-glucoside, and its malonyl derivative $(7.07 \%)$ decreased oxidative stress and inflammatory status when administered to C57BL/ 6 mice $(200 \mathrm{mg} / \mathrm{kg}$ ) fed with a high-fat diet (HFD) for 12 weeks. In fact, a reduced production of TNF- $\alpha$, IL-6, iNOS, and NF- $\kappa$ B in addition to a lower extent of hepatic and serum lipid peroxidation was registered, as demonstrated by a meaningful reduction of serum and hepatic triglyceride (TG), total cholesterol (TC), low density lipoprotein cholesterol (LDL-C), and malondialdehyde (MDA) levels respect to HFD control group. This extract also increased the total serum superoxide dismutase (SOD) and glutathione peroxidase (GPx) activities and reduced TC levels differently from black soybean extract which mainly contain petunidin-3-O-glucoside (69.89\%), delphinidin-3O-glucoside $(27.17 \%)$, and a very low amount of C3G, pelargonidin-3-O-glucoside, and peonidin-3-O-glucoside, probably due to the different composition in anthocyanins. Moreover, fatty acid decomposition is promoted and accelerated as evidenced by the increased fecal butyric acid level. Considering the overall effect of purple corn extract on mice, it could be considered very promising as agent preventing obesity via oxidative stress reduction [89]. Nixtamal treatment of blue hybrid corn increased SOD1 gene expression and total liver antioxidant capacity in rats fed a high-fat diet in respect to raw corn, thus statistically reducing liver inflammation [108].

The enhancing effects on the activity of SOD by anthocyanins have been known for over a decade; in fact, this action and the one on both mRNA expression and blood and urine oxidative status markers were monitored in sheep treated for 14 days with a purple corn anthocyanin-rich Japanese extract (consisting of 78\% anthocyanins, 20\% ethanol, and $2 \%$ citric acid, and added to animal diet at $0.5 \%$ anthocyanin concentration) in a crossover design [109]. The monitoring of urine 8-hydroxy-2'-deoxyguanosine (8-OHdG) and plasma MDA levels (markers of DNA and lipid oxidation, respectively) indicated that both treated and control animals did not undergo severe oxidative stress during the study, but a significant increase in plasma SOD activity was registered for the treated group, suggesting a positive mitigation activity of oxidative stress through the extract action on SOD activity. No variation in the main non-enzymatic antioxidants concentrations, i.e., total antioxidant capacity and total glutathione, was registered, differently from previous other researches testing the action of anthocyanins on these markers in monogastric animals [110], probably due to the poor absorption of the same anthocyanins by sheep. These results are promising, even if the researchers concluded that further studies are needed to understand the capacity of purple corn extract to counteract oxidative stress. Purple corn silage also increased blood SOD concentration $(9,333$ vs. $8,467 \mathrm{U} / \mathrm{mL})$ together with the milk production ( 31.7 vs. $29.2 \mathrm{~kg}$ /day) in lactating cows fed with rations containing $32 \%$ dry matter extract, ad libitum for 12 weeks, even if the anthocyanins concentration significantly decreased after 4 weeks of storage (70 mg/kg vs. $20 \mathrm{mg} / \mathrm{kg}$ dry matter) probably due to their degradation [111]. This positive effect on lactation was confirmed by Tian et al. [112] who demonstrated that the consumption of anthocyanin-rich purple corn stover silage by dairy goats for 7 days, after 14 days of adaptation to the new diet, increased not only the plasma antioxidant capacity (SOD activity), but also the levels of antioxidant gene expression (SOD2, Glutathione Peroxidase 1 (GPX1), and Glutathione Peroxidase 2 (GPX2)) while decreased mammary gland TNF mRNA expression in respect to normal diet goats. Moreover, a positive correlation between total antioxidant capacity and pelargonidin, SOD and peonidin, and SOD and malvidin-3-O-glucoside present in the extract was pointed out, demonstrating that anthocyanins can be transferred to goat milk [113].

\subsection{Effect on Memory and Neuroprotection}

The first report about the effect of the anthocyanin fraction present in blue corn tortillas on learning and memory capability was published by Aguirre López et al. [114]. In fact, adult rats fed with $6 \mathrm{~g}$ /day of blue corn tortillas for 38 days showed an improved learning and short-and long-term memory when assessed using Barnes's labyrinth at the end of the feeding period, differently from rats fed with white corn tortillas. In the same 
year, an extract obtained from purple waxy corn cob and pandan leaves was tested for its potential neuroprotective effect and memory enhancer in menopausal-model Wistar rats. For 28 days, rats were orally given 20,40 , and $80 \mathrm{mg} / \mathrm{kg}$ and non-spatial memory was evaluated using object recognition test every 7 days. In addition, oxidative stress status, acethylcolinesterase (AChE) inhibition effect, extracellular signal-regulated kinase 1/2 (ERK1/2) expression in the prefrontal cortex, and neuron density were evaluated at the end of the monitoring period. The lowest dose extract was able to reduce AChE activity, while an increasing in the ERK1/2 expression was evident for the dose $40 \mathrm{mg} / \mathrm{kg}$; neuron density and non-spatial memory improved at all the tested concentrations as well as the reduction of oxidative stress status [115]. The same extract, tested using the same animal model, also improved spatial memory through the suppression of AChE activity and neuron density in dentate gyrus of hippocampus through a better expression of ERK1/2. The registered effects globally enhanced the cognitive function as well as donezepil, a drug usually used in the treatment of memory impairment; therefore, this extract could be considered a good supplement at low cost, taking also into account that its LD50 was more than $2000 \mathrm{mg} / \mathrm{kg}$ body weight [116].

\subsection{Antiproliferative Effect}

Blue corn and tortilla extracts obtained using ethanol acidified with $1 \mathrm{M}$ citric acid were tested for their antiproliferative effects against hepatocellular carcinoma (HepG2), lung carcinoma (H-460), cervix adenocarcinoma (Hela), mammary adenocarcinoma (MCF-7), and prostate cancer androgen dependent (PC-3) using MTT assay. An antiproliferative effect was registered for all cell lines at $1000 \mu \mathrm{g}$ blue corn or tortilla extract $/ \mathrm{mL}$, with Hela cells being the most sensitive, suggesting that even if monomeric anthocyanin concentration can be reduced following the nixtamalization process in tortillas production, other active polymeric structures can be generated; this effect was also supported by the increase in antioxidant activity and by the same IC50 value of corn and tortilla extracts for the lipid peroxidation inhibition [87]. Purple and red corn extracts were also tested for their anti-proliferative effect on HCT-116 and HT-29 human colorectal cancer cells. Both the extracts suppressed angiogenesis and promoted apoptosis in the tested cell lines, as demonstrated by the reduction of Vascular Endothelial Growth Factor (VEGF) expression and angiopoietin receptor Tie-2 marker in HCT-116 and by their impact on markers of apoptosis (B-cell lymphoma 2 associated X protein ( Bcl2 associated X*BAX), B-cell lymphoma 2 (Bcl-2), cytochrome c, TRAILR2/D5) in HT-29 cells. Computational docking studies pointed out a negative value for free energies of bindings between anthocyanin fractions and receptor and non-receptor tyrosine kinases, thus indicating a spontaneous reaction and therefore the capability to suppress angiogenesis [84].

Recently, new nanoparticles obtained using purple waxy corn and blue butterfly pea petals extract were tested for their potential use in the treatment of cholangiocarcinoma (CCA), one of the poorest prognosis cancer, also resistant to the main chemotherapy drugs. A dose- and time-dependent anti-proliferative action of the new formulation was registered both in CCA cell line and in in a gemcitabine-resistant CCA cell line (KKU214GemR). Moreover, the efficacy of gemcitabine against KKU214GemR cells was enhanced by the co-treatment with low doses of extract, indicating a possible cytotoxic effect carried out by the downregulation of forkhead box protein (FOXM1), NF- $\mathrm{kB}, \mathrm{Bcl}-2$, and by induction of mitochondrial superoxide production [117].

\subsection{Other Effects}

An anthocyanin-enriched purple corn extract was tested against the development of orofacial allodynia induced in male rats by the injection of complete Freund's adjuvant, which caused temporomandibular joint (TMJ) inflammation. This extract, differently from the yellow corn extract, was able to prevent allodynia with efficacy very similar to that of acetyl salicylic acid when added to water starting 11 days before the beginning of the experiments and up to the end 3 days later, and only a partial additive effect was registered 
following the co-administration of the two agents. Purple corn extract possibly involved the reduction of trigeminal macrophage infiltration and the shift of microglia cell polarization to an anti-inflammatory phenotype, as demonstrated by a reduction in the expression of pro-inflammatory molecules, an increase in the production of anti-inflammatory mediators, and thinner and longer microglia cell processes which significantly contribute to neuronal pain [118].

Another interesting potential use of purple corn extract was tested in Wistar rat; a $50 \%$ extract cream was used to protect animals from the deleterious action of UV-B. The obtained results were interesting as when mice were exposed with UV-B $\left(840 \mathrm{~mJ} / \mathrm{cm}^{2}\right)$ for 4 weeks, a lower increase in matrix metalloproteinase-1 (MMP-1) level (an enzyme responsible for collagen breakdown in photoaging) and a reduced decrease of collagen amount in dermis were registered in mice group using purple corn cream with respect to the control group using a placebo cream [119].

A mixture of purple waxy corn cob and blue petal of Clitoria ternatea were incapsulated in niosome and incorporated into a mucoadhesive gel and tested for its capacity to act as a topical oral wound healing in vitro and in vivo. Anthocyanins created intra- and intermolecular interactions generating nano-sized clusters of crystalline-like aggregates in this system, thus improving permeation. Niosome gel (1\% and $10 \%)$ promoted the production of collagen in human gingival fibroblasts in in vitro test and was able to totally heal buccal wounds in Wistar rats after 5 days, possibly due to the improved mucosal permeability in addition to an anti-inflammatory effect exerted by the topic application [120].

The cardioprotective activity of an extract obtained by a new variety of corn rich in anthocyanin (a hybrid carrying the B1 and Pl1 alleles, which confer anthocyanin pigmentation) was evaluated in wild-type C57BL/6J mice, in which an intraperitoneal injection of $15 \mathrm{mg} / \mathrm{kg}$ body weight of doxorubicin (DOX), a chemotherapeutic agent commonly used in the treatment of blood, breast, ovarian, and lung cancers, induced cardiotoxicity. Mice were fed with a diet based on $29 \%$ of corn $(0.21 \pm 0.1 \mathrm{mg}$ anthocyanin/g powder) both for 3 weeks before and for 74 days after the induction of cardiotoxicity. A significant reduction of heart histopathological alterations in addition to a longer survival of treated animals than mice fed with normal diet was evident, confirming the previous results obtained in vitro on murine HL-1 cardiomyocytes pre-treated with C3G standard or purple corn extract, even if these treatments did not affect the cytotoxic effect of DOX on human cancer cell lines [121].

An aqueous extract of purple corn was also demonstrated to possess aphrodisiac activity when administered to male rats in the range $25-75 \mathrm{mg} / \mathrm{kg}$, by controlling ejaculation and by acting at the level of the central nervous system that control the brain and the spinal cord. In fact, the consumption of this extract significantly affected the arousal and execution of sexual behavior without significant influences on the ambulatory behavior and promoted an increase in the number of discharges of the ejaculatory motor [122].

Finally, an interesting work pointed out the effect of $5 \%$ purple corn extract supplementation of rainbow trout diet for eight weeks. A higher n-3 and n- 6 polyunsaturated fatty acids contents in fish body was registered as well as a lower adiposity; moreover, a higher gpx1 expression in erythrocytes and plasma total antioxidant capacity (TAC) indicated a potential effect against lipid peroxidation also in fish [123].

In Table 2, a summary of the in vitro and in vivo studies correlated with the health benefits discussed in the above section are reported. 
Table 2. A summary of the in vitro and in vivo studies correlated the healthy properties discussed in Section 5.

\begin{tabular}{|c|c|c|c|c|}
\hline Type of Trial & Metabolite, Origin & $\begin{array}{l}\text { Extract/Dose/Period } \\
\text { of Treatment }\end{array}$ & Main Biological Effect & Reference \\
\hline $\begin{array}{c}\text { In vitro anti-bacterial } \\
\text { activity (Lactobacillus } \\
\text { helveticus, } \\
\text { Bifidobacterium } \\
\text { longum, Helicobacter } \\
\text { pylori) }\end{array}$ & $\begin{array}{l}\text { Free and bound } \\
\text { phenolic compounds, } \\
\text { Peruvian purple corn }\end{array}$ & $\begin{array}{l}\text { free phenolic fraction } \\
10-50 \mathrm{mg} / \mathrm{mL} \text {, bound } \\
\text { phenolic fraction } \\
10 \mathrm{mg} / \mathrm{mL}\end{array}$ & No antibacterial activity & [79] \\
\hline $\begin{array}{l}\text { In vitro antibacterial } \\
\text { activity against } \\
\text { pathogenic bacteria } \\
\text { (Pseudomonas } \\
\text { aeruginosa, Escherichia } \\
\text { coli, Bacillus subtilis } \\
\text { and Staphylococcus } \\
\text { aureus) }\end{array}$ & $\begin{array}{l}\text { Polyphenolic extracts } \\
\text { from corn germplasm, } \\
\text { Chorotega and } \\
\text { Brunca Regions of } \\
\text { Costa Rica }\end{array}$ & $\begin{array}{l}100 \mathrm{mg} / \mathrm{mL} \\
\text { uncolored and } \\
\text { colored extracts }\end{array}$ & $\begin{array}{c}\text { Antibacterial activity of purple extract only } \\
\text { against } B \text {. subtilis }\end{array}$ & [96] \\
\hline $\begin{array}{c}\text { In vitro } \\
\text { antimutagenic } \\
\text { activity (TA100 and } \\
\text { TA98 Salmonella } \\
\text { typhimurium strains) }\end{array}$ & $\begin{array}{l}\text { Flavonoid and } \\
\text { anthocyanin extracts, } \\
\text { red and blue Mexican } \\
\text { corn }\end{array}$ & $\begin{array}{l}\text { Flavonoid extract: } 7 \\
\text { and } 138 \mathrm{~mm} \\
(+) \text {-catechin } \\
\text { equivalents, } \\
\text { anthocyanin extracts: } \\
1.6 \text { and } 3.2 \mathrm{~mm} \\
\text { cyanidin-3-glucoside } \\
\text { equivalents }\end{array}$ & $\begin{array}{l}\text { No effect on bacterial strain; } \\
\downarrow \text { Aflatoxin B1 mutagenicity }\end{array}$ & {$[38,97]$} \\
\hline $\begin{array}{l}\text { In vitro inhibitory } \\
\text { capacity of } \\
\text { hyperglycemia and } \\
\text { obesity target } \\
\text { enzymes }\end{array}$ & $\begin{array}{l}\text { Free phenolic fraction, } \\
\text { twenty-two Peruvian } \\
\text { corn samples } \\
\text { corresponding to five } \\
\text { corn races } \\
\text { (Arequipeño, } \\
\text { Cabanita, Kculli, } \\
\text { Granada and Coruca) }\end{array}$ & $\begin{array}{c}50,125,250 \mathrm{mg} \text { dose } \\
\text { for a-amylase assay; } \\
5,12.5,25 \mathrm{mg} \text { dose for } \\
\text { a -glucosidase assay; } \\
2.5,6.25,12.5 \mathrm{mg} \\
\text { dose for lipase assay }\end{array}$ & $\begin{array}{c}\uparrow \alpha \text {-glucosidase and a-amylase inhibitory } \\
\text { activity; only Kculli race sample } \uparrow \text { lipase } \\
\text { inhibitory activity }\end{array}$ & [100] \\
\hline $\begin{array}{l}\text { In vitro inhibitory } \\
\text { capacity of } \\
\text { hyperglycemia and } \\
\text { obesity target } \\
\text { enzymes }\end{array}$ & $\begin{array}{l}\text { Phytocomplex from } \\
\text { Moradyn purple corn } \\
\text { cob }\end{array}$ & $0.062-1 \mathrm{mg} / \mathrm{mL}$ & $\begin{array}{l}\uparrow \alpha \text {-glucosidase inhibitory activity; no } \\
\text { a-amylase inhibitory activity }\end{array}$ & [78] \\
\hline $\begin{array}{l}\text { In vitro response to } \\
\text { adipogenesis, } \\
\text { inflammation, and } \\
\text { insulin resistance in } \\
\text { 3T3-L1 adipocytes on } \\
\text { basal and } \\
\text { inflammatory } \\
\text { conditions }\end{array}$ & $\begin{array}{l}\text { Anthocyanin-rich } \\
\text { extract from purple } \\
\text { corn pericarp }\end{array}$ & $\begin{array}{l}0.4 \mathrm{mg} / \mathrm{mL}(50.3 \mu \mathrm{M} \\
\mathrm{C} 3 \mathrm{G} \text { equivalent })\end{array}$ & $\begin{array}{c}\downarrow \text { Preadipocyte differentiation, fatty acid } \\
\text { synthase enzymatic activity, PPAR- } \gamma \\
\text { transcriptional } \\
\text { activity on adipocytes in basal conditions } \\
\text { TNF- } \alpha \text {-dependent inflammatory status } \\
\text { ( } \uparrow \text { adiponectin, leptin), glucose uptake, and } \\
\text { GLUT4 membrane translocation, } \downarrow \text { reduced } \\
\text { reactive oxygen species generation }\end{array}$ & [80] \\
\hline $\begin{array}{l}\text { In vitro cellular } \\
\text { insulin secretion and } \\
\text { hepatic glucose } \\
\text { uptake (pancreatic } \\
\text { cells and hepatocytes) }\end{array}$ & $\begin{array}{l}\text { Anthocyanin-rich } \\
\text { extract from purple } \\
\text { corn pericarp }\end{array}$ & $0.125-1 \mathrm{mg} / \mathrm{mL}$ & $\begin{array}{c}\uparrow \text { Glucose-stimulated insulin secretion in } \\
\text { INS-1E cells and activation of the free fatty } \\
\text { acid receptor- } 1 \text {; } \\
\uparrow \text { glucose uptake in HepG } 2 \text { cells and } \\
\text { activation of glucokinase }\end{array}$ & [80] \\
\hline $\begin{array}{c}\text { In vitro } \\
\text { anti-inflammatory, } \\
\text { anti-adipogenic and } \\
\text { anti-diabetic activity } \\
\text { (mouse 3T3-L1 } \\
\text { preadipocytes and } \\
\text { RAW264.7 } \\
\text { macrophages) }\end{array}$ & $\begin{array}{l}\text { Anthocyanin-rich } \\
\text { extract from twenty } \\
\text { genotypes of Apache } \\
\text { Red purple corn } \\
\text { pericarp }\end{array}$ & $\begin{array}{c}0.05-1.0 \mathrm{mg} / \mathrm{mL} \text { in } \\
\alpha \text {-amylase and } \\
\text { dipeptidyl } \\
\text { peptidase- } 4 \\
\text { inhibitory assays; } \\
1 \mathrm{mg} / \mathrm{mL} \text { in the other } \\
\text { assays }\end{array}$ & $\begin{array}{c}\uparrow \text { Antiadipogenic effect by inhibiting } \\
\text { preadipocyte differentiation and reducing } \\
\text { TG content in mature adipocyte; } \\
\text { Inhibition of } \alpha \text {-amylase activity (IC50: } \\
\text { 109.5-172.7 } \mu \mathrm{g} / \mathrm{mL} \text { ), and DPP-IV activity } \\
\text { (IC50: } 65.5-702.7 \mu \mathrm{g} / \mathrm{mL} \text { ); } \\
\text { The presence of Cyanidin, perlagonidin } \\
\text { glycosylate forms, their malonilate } \\
\text { derivatives, quercetin, luteolin and vanillic } \\
\text { acid in corn extracts has been positively } \\
\text { correlated with anti-adipogenic potential }\end{array}$ & [82] \\
\hline
\end{tabular}


Table 2. Cont.

\begin{tabular}{|c|c|c|c|c|}
\hline Type of Trial & Metabolite, Origin & $\begin{array}{c}\text { Extract/Dose/Period } \\
\text { of Treatment }\end{array}$ & Main Biological Effect & Reference \\
\hline $\begin{array}{l}\text { In vitro anti-diabetic } \\
\text { activity }\end{array}$ & $\begin{array}{l}\text { Commercial purple } \\
\text { corn kernel extract }\end{array}$ & $\begin{array}{l}\text { IC50 } 26.12 \mu \mathrm{g} / \mathrm{mL} \text { for } \\
\text { phenolic acid and } \\
\text { flavonoids fraction } \\
\text { IC50 } 58.20 \mu \mathrm{g} / \mathrm{mL} \text { for } \\
\text { anthocyanin fraction }\end{array}$ & $\downarrow$ Tyrosine phosphatase $1 \beta$ expression & {$[61]$} \\
\hline $\begin{array}{l}\text { In vitro anti-obesity } \\
\text { activity (murine } \\
\text { 3T3-L1 cell line) }\end{array}$ & $\begin{array}{l}\text { Purple corn silk } \\
\text { extract }\end{array}$ & $250-1000 \mu \mathrm{g} / \mathrm{mL}$ & $\begin{array}{l}\downarrow \text { Adipogenesis (inhibition on adipocyte } \\
\text { differentiation and reduction of total lipid } \\
\text { accumulation); } \\
\uparrow \text { Lipolysis and apoptosis inductive effect } \\
\text { (increase of released glycerol content and of } \\
\text { nucleic condensing and apoptotic bodies) }\end{array}$ & [26] \\
\hline $\begin{array}{l}\text { In vivo (C57BL/KsJ } \\
\mathrm{db} / \mathrm{db} \text { mice }) \\
\text { anti-diabetic effect }\end{array}$ & $\begin{array}{l}\text { Anthocyanin-rich } \\
\text { purple corn extract }\end{array}$ & $\begin{array}{l}10 \text { or } 50 \mathrm{mg} / \mathrm{kg} / \text { day } \\
\text { for } 8 \text { weeks }\end{array}$ & $\begin{array}{c}\text { Protection of pancreatic } \beta \text {-cells, increase of } \\
\text { insulin secretion and AMPK activation in } \\
\text { the liver } \\
\text { ( } \downarrow \text { blood glucose and HbA1c levels, } \uparrow \\
\text { C-peptide and adiponectin levels) }\end{array}$ & [79] \\
\hline $\begin{array}{l}\text { In vivo (rats) } \\
\text { attenuation of } \\
\text { diet-induced } \\
\text { metabolic syndrome }\end{array}$ & $\begin{array}{l}\text { Purple corn kernel } \\
\text { flour }\end{array}$ & $\begin{array}{l}50 \mathrm{mg} / \mathrm{kg} / \text { die } \\
(\approx 8 \mathrm{mg} \text { antho- } \\
\text { cyanins } / \mathrm{kg} / \text { die }) \text { with } \\
\text { or without high fat or } \\
\text { high carbohydrate } \\
\text { diet for } 8 \text { weeks, after } \\
8 \text { weeks of only high } \\
\text { fat or high } \\
\text { carbohydrate diets }\end{array}$ & $\begin{array}{c}\downarrow \text { Visceral adiposity index, total body fat } \\
\text { mass, systolic blood pressure, plasma } \\
\text { triacylglycerols, total cholesterol, blood } \\
\text { glucose, HbA1c, and glucagone; improved } \\
\text { glucose tolerance, liver, and cardiovascular } \\
\text { structure and function }\end{array}$ & [103] \\
\hline $\begin{array}{l}\text { In vivo (Wistar rats) } \\
\text { attenuation of } \\
\text { diet-induced } \\
\text { metabolic syndrome }\end{array}$ & Blue corn extract & $\begin{array}{l}2 \mathrm{~mL} / \text { die }(9.97 \mathrm{mg} \\
\text { total polyphenols of } \\
\text { which } 2.92 \mathrm{mg} \text { of } \\
\text { anthocyanins) with or } \\
\text { without high-sucrose } \\
\text { diet or high-sucrose } \\
\text { diet plus cholesterol, } \\
\text { for } 4 \text { weeks after } 12 \\
\text { weeks of } \\
\text { high-sucrose diet }\end{array}$ & $\begin{array}{c}\uparrow \text { Enhanced HDL-C; } \\
\downarrow \text { Systolic blood pressure, serum } \\
\text { triglycerides, total cholesterol, epididymal } \\
\text { adipose tissue weight }\end{array}$ & [104] \\
\hline $\begin{array}{l}\text { In vivo (Sprague } \\
\text { Dawley rats) } \\
\text { amelioration of lipid } \\
\text { metabolism }\end{array}$ & Purple corn extract & $\begin{array}{l}401 \mathrm{mg} \text { of } \\
\text { anthocyanins } \\
\text { per food } \mathrm{kg} \text {, with } \\
\text { olive or chia oil for } 78 \\
\text { days }\end{array}$ & $\begin{array}{c}\uparrow \text { Anti-adipogenic effect mediated by the } \\
\text { upregulation of liver PPAR- } \alpha \text { and the } \\
\text { inhibition of SREPB-1C following the } \\
\text { combined intake of Purple corn extract and } \\
\text { Chia oil }\end{array}$ & [105] \\
\hline $\begin{array}{l}\text { In silico study to } \\
\text { evaluate anti-diabetic } \\
\text { effect }\end{array}$ & Blue corn extract & $\begin{array}{l}\text { Total anthocyanins: } \\
582 \mathrm{mg} / \mathrm{kg} \text { DW } \\
\text { (C3G) }\end{array}$ & 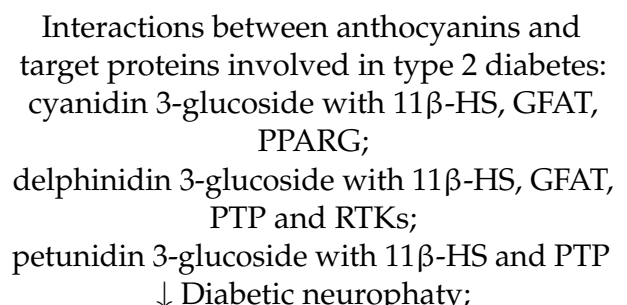 & [86] \\
\hline $\begin{array}{l}\text { In vivo } \\
\text { (streptozotocin- } \\
\text { diabetic rats) effect on } \\
\text { oxidative stress } \\
\text { related disordersin } \\
\text { diabetic condition }\end{array}$ & $\begin{array}{l}\text { Zingiber officinale } \\
\text { rhizomes and purple } \\
\text { Zea mays seeds } \\
\text { extract }(1: 4, v / v)\end{array}$ & $\begin{array}{l}100-300 \mathrm{mg} / \mathrm{kg} / \mathrm{die} \\
\text { for } 21 \text { days }\end{array}$ & $\begin{array}{c}\text { Improved sciatic function index, paw } \\
\text { withdrawal threshold intensity, paw } \\
\text { withdrawal } \\
\text { latency, nerve conduction velocity); } \\
\downarrow \text { Oxidative stress status } \\
\text { (MDA, SOD, GPx, CAT, AR); } \\
\uparrow \text { Axon density in the lesion nerve. }\end{array}$ & [106] \\
\hline
\end{tabular}


Table 2. Cont.

\begin{tabular}{|c|c|c|c|c|}
\hline Type of Trial & Metabolite, Origin & $\begin{array}{c}\text { Extract/Dose/Period } \\
\text { of Treatment }\end{array}$ & Main Biological Effect & Reference \\
\hline $\begin{array}{l}\text { In vitro } \\
\text { anti-inflammatory } \\
\text { properties }\end{array}$ & $\begin{array}{l}\text { Wet milling, dry } \\
\text { milling, and dry } \\
\text { grind purple and red } \\
\text { corn pericarp }\end{array}$ & $\begin{array}{l}1 \mathrm{mg} / \mathrm{mL} \text { red corn } \\
\text { dry milling pericarp } \\
\text { proanthocyanidin } \\
0.1-1 \mathrm{mg} / \mathrm{mL} \text { purple } \\
\text { corn dry milling } \\
\text { pericarp } \\
\text { proanthocyanidin }\end{array}$ & $\begin{array}{c}\downarrow \text { Cyclooxygenase-2; } \\
\downarrow \text { Inducible nitric oxide synthase } \\
\text { activities }\end{array}$ & [85] \\
\hline $\begin{array}{l}\text { In vitro effect on } \\
\text { inflammatory } \\
\text { paracrineinterplay } \\
\text { between } \\
\text { macrophages and } \\
\text { adipocytes }\end{array}$ & $\begin{array}{l}\text { Dry-milling purple or } \\
\text { red corn pericarp } \\
\text { extract }\end{array}$ & $\begin{array}{l}0.25-1 \mathrm{mg} / \mathrm{mL} \\
\text { purple or red } \\
\text { pericarp extract }\end{array}$ & $\begin{array}{c}\downarrow \text { Reduction of pro-inflammatory mediators } \\
\text { expressed by LPS-induced magrophages or } \\
\text { adipocytes exposed to } \\
\text { macrophage-conditioned medium; } \\
\downarrow \text { Inhibition of NF- } \mathrm{B} \text { and JNK } \\
\text { pro-inflammatory pathways; } \\
\downarrow \text { Insulin resistance caused by } \\
\text { adipocyte-macrophage interaction under a } \\
\text { metaflammation state }\end{array}$ & [107] \\
\hline $\begin{array}{l}\text { In vivo (C57BL/ } 6 \\
\text { mice) effect on } \\
\text { oxidative stress and } \\
\text { inflammatory status } \\
\text { associated to obesity }\end{array}$ & Purple corn extract & $\begin{array}{l}200 \mathrm{mg} / \mathrm{kg} \text { with a } \\
\text { high-fat diet, } 12 \\
\text { weeks }\end{array}$ & $\begin{array}{c}\downarrow \text { serum and hepatic lipid peroxidation (TG, } \\
\text { TC, LDL, MDA, SOD, TNF } \alpha \text {, IL-6, iNOS, } \\
\text { NF- } k B, \text { GPx); } \\
\uparrow \text { fecal butyric acid levels }\end{array}$ & [89] \\
\hline $\begin{array}{l}\text { In vivo (rats) effect on } \\
\text { liver oxidative stress }\end{array}$ & $\begin{array}{l}\text { Raw and nixtamal } \\
\text { blue corn }\end{array}$ & $\begin{array}{l}18 \mathrm{~g} / \text { day, with and } \\
\text { without high fat diet, } \\
8 \text { weeks }\end{array}$ & $\begin{array}{l}\downarrow \text { Oxidative stress (MDA, ROS, SOD, GPx, } \\
\text { CAT) }\end{array}$ & [108] \\
\hline $\begin{array}{l}\text { In vivo (sheep) effect } \\
\text { on oxidative stress }\end{array}$ & $\begin{array}{l}\text { Sunred NO.5 F } \\
\text { purple corn pigment }\end{array}$ & $\begin{array}{l}\text { Diet: } 78 \% \text { pigment, } \\
30 \% \text { EtOH, } 2 \% \text { citric } \\
\text { acid, } 14 \text { days }\end{array}$ & $\begin{array}{l}\downarrow \text { Serum lipid peroxidation (SOD, total } \\
\text { antioxidant capacity, GSH, 8-HOdG); } \\
\downarrow \text { Oxidation resistance of plasma }\end{array}$ & [109] \\
\hline $\begin{array}{l}\text { In vivo (cows) effect } \\
\text { on oxidative stress } \\
\text { and lactation }\end{array}$ & Purple corn silage & $\begin{array}{l}32 \% \text { dry matter } \\
\text { extract, ad libitum, } 12 \\
\text { weeks }\end{array}$ & $\begin{array}{l}\downarrow \text { Oxidative stress (SOD); } \\
\quad \uparrow \text { Milk production }\end{array}$ & [111] \\
\hline $\begin{array}{l}\text { In vivo (dairy goats) } \\
\text { effect on oxidative } \\
\text { stress and lactation }\end{array}$ & $\begin{array}{l}\text { Commercial } \\
\text { anthocyanin-rich } \\
\text { purple corn pigment, } \\
\text { Anthocyanin-rich } \\
\text { purple corn stover } \\
\text { silage }\end{array}$ & $1 \mathrm{~g} /$ die, 7 days & $\begin{array}{c}\downarrow \text { Oxidative stress (SOD, GPX2); } \\
\uparrow \text { Milk production (TNF, mRNA expression) }\end{array}$ & [112] \\
\hline $\begin{array}{l}\text { In vivo (dairy goats) } \\
\text { effect on oxidative } \\
\text { stress and lactation }\end{array}$ & $\begin{array}{l}\text { Anthocyanin-rich } \\
\text { purple corn stover } \\
\text { silage }\end{array}$ & $\begin{array}{l}862 \mathrm{mg} / \mathrm{kg} \text { dry } \\
\text { matter, } 6 \text { weeks }\end{array}$ & $\begin{array}{c}\downarrow \text { Oxidative stress (SOD, total antioxidant } \\
\text { capacity); } \\
\uparrow \text { Milk production }\end{array}$ & [113] \\
\hline $\begin{array}{l}\text { In vivo (rats) effect } \\
\text { on learning and } \\
\text { memory capability }\end{array}$ & Blue corn tortilla & $6 \mathrm{~g} /$ day, 28 days & $\begin{array}{l}\uparrow \text { Learning and short-and long-term } \\
\text { memory (Barnes's labyrinth test) }\end{array}$ & [114] \\
\hline $\begin{array}{l}\text { In vivo } \\
\text { (menopausal-model } \\
\text { Wistar rats) } \\
\text { neuroprotective effect }\end{array}$ & $\begin{array}{l}\text { Purple waxy corn cob } \\
\text { and pandan leaves } \\
\text { extract }(1: 1, w / w)\end{array}$ & $\begin{array}{c}20,40,80 \mathrm{mg} / \mathrm{kg}, 28 \\
\text { days }\end{array}$ & $\begin{array}{c}\uparrow \text { Non-spatial memory (ERK1/2, neuron } \\
\text { density); } \\
\downarrow \text { Oxidative stress status (AChE) }\end{array}$ & [115] \\
\hline $\begin{array}{l}\text { In vivo } \\
\text { (menopausal-model } \\
\text { Wistar rats) effect on } \\
\text { cognitive function } \\
\text { In vitro cellular }\end{array}$ & $\begin{array}{l}\text { Purple waxy corn cob } \\
\text { and pandan leaves } \\
\text { extract }(1: 1, w / w)\end{array}$ & $\begin{array}{c}20,40,80 \mathrm{mg} / \mathrm{kg}, 28 \\
\text { days }\end{array}$ & $\begin{array}{c}\uparrow \text { Spatial memory (ERK1/2, neuron } \\
\text { density); } \\
\downarrow \text { Oxidative stress status (AChE) }\end{array}$ & [116] \\
\hline $\begin{array}{l}\text { anti-cancer properties } \\
\text { (HepG2, H-460, Hela, } \\
\text { MCF-7, PC-3) and } \\
\text { antioxidant effect }\end{array}$ & $\begin{array}{l}\text { Blue corn and tortilla } \\
\text { extracts }\end{array}$ & $1 \mathrm{mg} / \mathrm{mL}$ & $\begin{array}{l}\uparrow \text { Anti-proliferative effect (MTT assays); } \\
\uparrow \text { Antioxidant activity (DPPH, TBARS) }\end{array}$ & [87] \\
\hline
\end{tabular}


Table 2. Cont.

\begin{tabular}{|c|c|c|c|c|}
\hline Type of Trial & Metabolite, Origin & $\begin{array}{c}\text { Extract/Dose/Period } \\
\text { of Treatment }\end{array}$ & Main Biological Effect & Reference \\
\hline $\begin{array}{l}\text { In vitro cellular } \\
\text { anti-cancer properties } \\
\text { (HCT-116, HT-29) }\end{array}$ & $\begin{array}{l}\text { Purple and red corn } \\
\text { extracts }\end{array}$ & $0.25-10 \mathrm{mg} / \mathrm{mL}$ & $\begin{array}{c}\downarrow \text { Angiogenesis (VEGF expression, Tie-2 } \\
\text { marker); } \\
\uparrow \text { Apoptosis (BCL2 Associated X*BAX, } \\
\text { B-cell lymphoma 2-Bcl-2, cytochrome c, } \\
\text { TRAILR2/D5) }\end{array}$ & [84] \\
\hline $\begin{array}{l}\text { In vitro cellular } \\
\text { anti-proliferative } \\
\text { effect (CCA and } \\
\text { KKU214GemR cell } \\
\text { lines) }\end{array}$ & $\begin{array}{l}\text { Anthocyanin } \\
\text { complex (purple } \\
\text { waxy corn and blue } \\
\text { butterfly pea petals) }\end{array}$ & $100-800 \mu \mathrm{g} / \mathrm{mL}$ & $\begin{array}{l}\uparrow \text { Citotoxicity effect (FOXM1, NF- } \mathrm{BB}, \mathrm{Bcl}-2, \\
\text { mitochondrial superoxide production) }\end{array}$ & [117] \\
\hline $\begin{array}{l}\text { In vivo (male rats) } \\
\text { anti- orofacial } \\
\text { allodynia effect }\end{array}$ & $\begin{array}{l}\text { Anthocyanin- } \\
\text { enriched purple corn } \\
\text { extract (titrated } 4 \% \\
\text { anthocyanin) }\end{array}$ & $\begin{array}{l}53 \mathrm{mg} \\
\text { anthocyanins } / \mathrm{kg} \\
\text { body, } 11 \text { days before } \\
\text { and } 3 \text { day after } \\
\text { experiments }\end{array}$ & $\begin{array}{c}\downarrow \text { Trigeminal macrophage infiltration } \\
\text { (modulation of microglia reactivity); } \\
\uparrow \text { Von Frey Behavioral Test }\end{array}$ & [118] \\
\hline $\begin{array}{l}\text { In vivo (mice) skin } \\
\text { protection }\end{array}$ & Purple corn & $\begin{array}{c}50 \% \text { purple corn } \\
\text { cream, } 4 \text { weeks, twice } \\
\text { a day }\end{array}$ & $\downarrow$ Photoaging (MMP-1, collagen in dermis) & [119] \\
\hline $\begin{array}{l}\text { In vitro (human } \\
\text { gingival fibroblast) } \\
\text { and in vivo (rats) } \\
\text { wound healing }\end{array}$ & $\begin{array}{l}\text { Purple waxy corn cob } \\
\text { and Clitoria ternatea } \\
\text { blue petals extract }\end{array}$ & $\begin{array}{l}1 \text { or } 10 \% w / w \text { in } \\
\text { niosomes }\end{array}$ & $\uparrow$ in vitro permeation and wound healing & [120] \\
\hline $\begin{array}{l}\text { In vitro effect on cell } \\
\text { survival (murine } \\
\text { HL-1 cardiomyocytes) } \\
\text { In vivo (C57BL/6J } \\
\text { mice) } \\
\text { cardioprotective } \\
\text { effect }\end{array}$ & $\begin{array}{l}\text { Cyanidin-3-glucoside } \\
\text { Anthocyanin-rich B1 } \\
\text { P11 hybrid seeds } \\
\text { powder }\end{array}$ & $\begin{array}{c}125 \mathrm{mM} \\
\text { Diet based on } 29 \% \text { of } \\
\text { corn }(0.21 \pm 0.1 \mathrm{mg} \\
\text { anthocyanin } / \mathrm{g} \\
\text { powder }), 3 \text { weeks } \\
\text { before- } 74 \text { days after } \\
\text { the induction of } \\
\text { cardiotoxicity with } \\
\text { doxorubicin }\end{array}$ & $\begin{array}{c}\uparrow \text { Cell viability; } \\
\uparrow \text { Animal survival; } \\
\downarrow \text { Histopathological alterations }\end{array}$ & [121] \\
\hline $\begin{array}{l}\text { In vivo (rats) } \\
\text { aphrodisiac activity }\end{array}$ & Purple corn extract & $\begin{array}{l}25-75 \mathrm{mg} / \mathrm{kg} \text { single } \\
\text { oral treatment }\end{array}$ & $\begin{array}{l}\text { Facilitates the arousal and execution of } \\
\text { sexual behavior; } \\
\uparrow \text { Number of discharges of the ejaculatory } \\
\text { motor patterns and total number of genital } \\
\text { motor patterns }\end{array}$ & [122] \\
\hline $\begin{array}{l}\text { In vivo (rainbow } \\
\text { trout) antioxidant } \\
\text { effect and lipid } \\
\text { peroxidation } \\
\text { inhibition }\end{array}$ & Purple corn extract & $\begin{array}{l}5 \% \text { extract feeding, } \\
\text { twice a day, } 8 \text { weeks }\end{array}$ & $\begin{array}{c}\uparrow \text { Antioxidant activity (gpx1 enzyme, TAC, } \\
\text { n-3 and n-6 PUFA) }\end{array}$ & [123] \\
\hline
\end{tabular}

\section{Colored Corn-Based Foods and Technological Aspects}

Technological processes and thermal treatment applied in the different cooking methods highly affected colored corn-based food. In particular, thermal treatment caused significant decreases in polyphenolic compounds concentration and antioxidant activity; moreover, sometimes it altered the polyphenolic composition. The type of cooking is also a key factor because generally boiling caused a more sensitive decrease in corn antioxidants than steam cooking, and anthocyanins as well as other phenolic compounds are lost into the cooking water which could be considered a valuable co-product. A greater loss of healthy compounds was registered for kernels removed from the cob prior cooking, differently from kernels cooked on the ear [83].

The use of corn flour in the design of gluten-free products has been recently increased thanks to several studies on the doughs rheological behavior and on the parameters 
affecting such doughs (i.e., particle size, temperature, water absorption, and shear rate), which are fundamental for the selection of the technological conditions. Corn variety and milling conditions highly affected chemical characteristics of flours and rheological properties of doughs; in fact, the particle size of yellow and purple corn flours were generally smaller than those obtained for white flour when the same milling process was applied and the water absorption as well as the damage to starch present in corn increased. Moreover, elastic behavior was predominant in relation to viscous component and the viscoelastic moduli values decreased. The rheological characterization results suggested that dough properties were strongly related to processing and raw corn characteristics [124]. Moreover, purple genotypes had higher gelatinization enthalpy than non-purple ones due to the fact that anthocyanins increased starch granules fragility during cooking (these genotypes had a lower amylose content in relation to non-purple), as demonstrated by Sebastián Mansilla et al. [125] for purple maize flour from Argentina. In addition, a negative correlation between anthocyanins content and the amylopectin retrogradation enthalpy was evident, probably due to interactions formed after starch gelatinization. Interesting results were obtained by Camelo-Méndez et al. [126] who evaluated the addition of different amount of blue corn flour to composite pasta containing chickpea and unripe plantain in order to develop a gluten-free product. The final product was characterized by a dark color and a high polyphenols content (especially pasta containing $75 \%$ blue flour) also after extrusion ( $20 \%$ lost) and cooking ( $30 \%$ lost), and by higher adhesiveness and lower hardness and chewiness than the corresponding white corn-based pasta. The extrusion process was responsible for a lower peak viscosity than the corresponding raw product, more marked for blue flour pasta than the corresponding white, due to the disorganization of starch during the process; however, a second peak viscosity was registered upon cooling for blue pasta indicating a re-arrangement of starch components which could contribute to the retention of polyphenols. Another example of blue corn use in extruded snacks was that relative the preparation containing orange bagasse (the main waste deriving from juice preparation) as a fiber source. Orange bagasse also made hard the extrudates (as showed by the reduction of expansion index) which were characterized by a high numbers per area and small size pores. In addition, starch lost its semicrystalline structure as a consequence of high temperature and mechanical shearing, as showed by infrared spectra and X-ray diffraction patterns [127]. The potential use of purple corn flours in designing of gluten-free eggless muffins without gum addition was demonstrated as well as of white and yellow accessions. The low breakdown viscosity indicated a thermal stability for all the tested accession, even if they differed for physicochemical, antioxidant, and pasting properties. A positive correlation between cohesiveness and chewiness was registered; conversely, a negative correlation with paste viscosities was undelighted. Sensory analysis of muffins prepared from purple corn revealed that they were not so acceptable, differently from those prepared from yellow accessions. These preparations are promising, especially considering the fact that they are eggless and their use could be helpful to counteract the increase in incidences of egg allergies [21].

Common Latin American dishes are based on masa, obtained by the nixtamalization process consisting of an alkali thermal treatment of corn to obtain a dough or a flour (nixtamalized corn flour) after a drying process which reduces water content to $8-10 \%$. This process highly affects thermal, physicochemical, sensory, and also nutritional properties of nixtamalized products [128]. In fact, a high protein content, calcium, and fiber characterized tortillas, tortillas chips, and other Mexican food produced using masa and nixtamalized flour. Moreover, corn wet milling is easier because of the germ and endosperm absorption of water and calcium and gums are released by pericarp and germ during washing phase of the process, thus reducing the cohesive and adhesive properties of the masa $[129,130]$. Significant differences in the physical properties of different blue corn varieties were registered during nixtamalization process, such as shorter cooking times for the softer kernels. In such conditions, consistency and stickiness masa and hardness and extensibility of tortillas overlap with those of the hard (flint and dent) varieties. Moreover, 
a reduction in yellow and reddish tones and an enhance in green tones was registered following nixtamalization and all tested flour varieties showed similar gelatinization temperatures. Conversely, dent and flint varieties of flours had the greatest enthalpy values due to a more organized kernel structure. Rheological and thermal parameters indicated that generally the Southwest floury varieties are more suitable for tortilla production [131]. The traditional nixtamalization and lime-cooking extrusion process more markedly decreased carotenoids concentration and the oxygen radical absorbance capacity (ORAC) in red Mexican pigmented corns than in yellow varieties. Only lutein concentration was less affected in traditional and extruded tortillas [132]. Different nutritional value and bioactive compounds concentrations were registered for artisan handmade and commercial/industrial tortillas prepared using white and blue corn. Commercially produced white tortillas had a lower antioxidant capacity, fiber, calcium, and free polyphenols content than blue handmade ones; in particular, blue handmade tortillas had 4.5-fold ferulic acid content compared with white commercially produced. Therefore, artisan fresh tortillas had superior nutritional-nutraceutical properties compared to white commercial products [133]. Huitlacoche (Ustilago maydis DC Corda) is a fungus traditionally consumed in nixtamalized products as it affected corn for two months a year, generating smut on ear, stem, and leaves, and it is a good source of nutraceuticals as well as protein, fiber, and water. Its addition to nixtamalized blue corn flours at different percentages (from $3 \%$ to $18 \%$ ) significantly affected color, water-absorption, adhesiveness, and, in general, the rheological properties of flour, as recently demonstrated by Amador-Rodríguez et al. [134]. In particular, an addition of 3-9\% exhibited good machinability in an industrial tortilladora machine, and therefore, huitlacoche could be used as a healthier ingredient in the production of food.

Blue corn varieties are well acceptable when used in the production of popcorn, chips, and flour-based confectionary not only for the attractive color, but also because they have important nutritional and healthy properties due to their high anthocyanin and fiber contents, as confirmed in a recent investigation on Turkey varieties. In fact, $61.12{ }^{\circ} \mathrm{C}$, $64.35^{\circ} \mathrm{C}$, and $75.65^{\circ} \mathrm{C}$ were the temperature values registered for the gelatinization onset, peak, and end, respectively. Considering their nutritional composition, the mean contents of moisture, lipid (with oleic, linoleic, palmitic, and stearic the main fatty acids), total protein, and crude fiber were $9.39 \%, 4.30 \%, 13.13 \%$, and $2.68 \%$, respectively. Total starch and resistant starch were $63.94 \%$ and $8.89 \%$, respectively [ 43 ].

Another interesting use was in the production of polvorones, a typical energetic and nutritional Mexican bakery product. This food had better color, flavor, and acceptability than that produced using wheat flour. In addition, the presence of anthocyanins highlighted healthy beneficial properties as the only degradation registered during the transformation of raw flour are the de-acylation of some acylated anthocyanins [88].

A combination of purple corn and rice flours as substitute of wheat flour in bakery product was also investigated. A ratio of 71.1:28.9 ensured the highest anthocyanin concentration and antiradical activity, and the best texture score of purple corn cake containing corn silk. Its acidification with 3\% fumaric acid preserved the color and polyphenols degradation and therefore this product could be considered a high nutritional and healthy food [135].

As regards the production of liquid food, a modified "Sendechó" beverage was proposed by Flores-Calderón et al. [136] in 2017 who added hops and brewer's yeast to guajillo chili and blue corn malt (the traditional ingredients), thus obtaining a beverage with the same characteristics of Sendechó in addition to that of corn beer due to an ale fermentation process. Different levels of hops, guajillo, and the addition of caramel malt to blue corn malt were used to prepare eight blue corn malt beers tested for their total acidity, bitterness units, total reducing sugars, alcohol, cis- and trans-iso- $\alpha$-acids concentration, total anthocyanins and polyphenols, as well as their scavenging activity against ABTS radical cation and DPPH. All these parameters were monitoring during three different stages of the process, i.e., boiling-wort, fermentation - green beer, and maturation-mature beer, and the results indicated that all, with the exception of $\mathrm{pH}$, total acidity, and alcohol 
content, were affected especially in mature beer. In fact, a marked decrease in polyphenolic concentration and antioxidant activity was registered. However, low-alcohol beers with $\mathrm{pH}$, total acidity, bitterness units, iso- $\alpha$-acids level, and total reducing sugars similar to barley beers were obtained. Beers based on $85 \%$ corn malt and $15 \%$ caramel malt showed the highest anthocyanins content and scavenging activity against ABTS radical. Peruvian Andean corn variety, typical of a limited Peruvian zones, is used to prepare a drink, namely chicha morada, containing an extract of the purple pigments, fruits, such as pineapple, quince, apple, and peach, which conferred flavors, and a starch source (potato, corn, or sweet potato). If a cooking process is applied and dried or fresh fruits are added, mazamorra morada, a dessert, is obtained. This particular variety had important nutritional and health effects and its flour can also be used in other foods, (pasta, bread, and cakes), providing color and flavor to the processed products [137].

The addition of $0.3 \%$ purple corn pigment containing pelargonidin, peonidin, cyanidin, malvidin, petunidin, and delphinidin to milk samples resulted in a retarding action on unsaturated fatty acids oxidation during a 7 day milk storage period. Anthocyanins could inhibit the oxidation of Unsaturated Fatty Acids (UFAs) by chain radical termination, acting as hydrogen atom donors to the peroxy radical; specifically, pelargonidin and petunidin significantly affected C14: $1 n-5$, C17: $1 n-7$, C18: $2 n-6$, C20: 2n-6, C20: 3n-3, and C20: 4n-6 degradation as well as cyanidin and total anthocyanins affected C14: 1n-5, C16: 1n-7, C17: $1 n-7$ [138].

Finally, recent studies were performed in order to obtain anthocyanin fractions from colored corns for the production of functional foods with higher resistant starch content. Blue corn extract, rich in cyanidin-3-O-(6"-O-malonylglucoside) was able to modify the relation of starch fractions of commercial maize starch contributing to a higher resistant starch formation, probably due to anthocyanins-starch interactions. Therefore, the amylolytic enzymatic hydrolysis in the small intestine could be limited and anthocyanins could be released in colon where the fermentation process led to molecules able to prevent colon cancer [139]. Similar results were also obtained testing flours from three different pigmented varieties when cooked. In fact, after in vitro starch digestion step higher resistant starch content was detected in colored samples in respect to a yellow variety, as well as lower starch hydrolysis index. Moreover, in this research, anthocyanins have been indicated as compounds able to modulate the enzyme activity and starch digestibility [140].

\section{Non-Food Uses}

Pigmented corn can be used in dry-grind ethanol process both in conventional starch hydrolyzing enzymes and in granular starch hydrolyzing enzymes process without any negative effect of the fermentation, as reported by Wang et al. [141] in 2016. In fact, for pigmented varieties similar ethanol conversion efficiencies to those of yellow corn were registered $(75.1 \pm 0.2 \%$ for yellow dent corn, $74.3 \pm 0.4 \%$ for red corn, $78.4 \pm 0.5 \%$ for blue corn, and $81.2 \pm 1.0 \%$ for purple corn). Different results were obtained by Somavat et al. [20]: In fact, $14.3 \%(v / v)$ vs. $17.2 \%(v / v)$ final ethanol concentration were obtained for blue corn and yellow dent corn, respectively, in dry-grind process. Wet-milling and dry-grind characteristics of pigmented corn were also considered. In wet-milling, lower starch yields were registered for purple and blue corn than for yellow dent corn and in dry-milling, blue corn gave the lowest total endosperm yield. Anyway, purple and blue corn can potentially be used in all the three milling processes.

Ethanol production was estimated 42,37, and 35.2 million gallon/yr for yellow, blue, and purple corn, respectively, as reported by Somavat et al. [142] after a commercial scale economic and technical feasibility analysis of dry grind process using pigmented corn to produce simultaneously ethanol and anthocyanins.

Purple corn extract was also used to develop an antimicrobial food packaging film. It was incorporated together with silver nanoparticles (AgNPs) into chitosan to develop active and intelligent food packaging films through hydrogen bonds (differently from silver nanoparticles which linked to chitosan by a coordination effect). Chitosan/AgNPs/purple 
corn extract film exhibited a higher barrier than chitosan/AgNPs or chitosan/purple corn extract. High antioxidant and antimicrobial properties were also registered due to synergistic effect between corn extract and AgNPs. The only disadvantage could be the changing color of films depending on $\mathrm{pH}$ value because of high level in anthocyanins present in purple corn extract (PCE). Anyway, chitosan/AgNPs/PCE film could be considered a novel active and intelligent food packaging material in food industry [143].

Another non-food interesting use of pigmented corn is the use of the anthocyanin fraction from husk, cob and silk of purple corn as photosensitizers in dye sensitized solar cells. The highest efficiency was obtained from purple corn husk extracted by acetone. The main characteristics of these solar cells are the high performance, the low cost, and the fact that they are environmentally friendly [144].

\section{Conclusions}

In the last years, the use of pigmented corns and the recycle of their waste represented an important issue for human health. In fact, many secondary metabolites belonging to different chemical classes of compounds have been detected in colored corn (seed, kernel, corn, husk, and bran derived-products) using chromatographic techniques and also many innovative approaches for the extraction of these molecules have been set up. The stability of the extracts deriving from colored corns is a key point in their potential use as food-derived products and this review also highlighted the different approached used to stabilize them. Finally, the main bioactivities registered in vitro and in vivo in animal models have been discussed.

Author Contributions: Conceptualization, A.P.; methodology, A.P. and R.C.; investigation, L.F.; writing—original draft preparation, A.P., R.C. and L.F.; writing—review and editing, A.P.; supervision, A.P. All authors have read and agreed to the published version of the manuscript.

Funding: This research received no external funding.

Conflicts of Interest: The authors declare no conflict of interest.

\section{References and Notes}

1. FAOSTAT. Available online: http://www.fao.org/faostat/en/\#data/QC (accessed on 12 November 2020).

2. Afzal, M.; Redha, A.; Al Hasan, R. Anthocyanins potentially contribute to defense against Alzheimer's disease. Molecules 2019, 24, 4255. [CrossRef] [PubMed]

3. de Sousa Moraes, L.F.; Sun, X.; Peluzio, M.D.C.G.; Zhu, M.J. Anthocyanins/anthocyanidins and colorectal cancer: What is behind the scenes? Crit. Rev. Food Sci. Nutr. 2019, 59, 59-71. [CrossRef] [PubMed]

4. Krga, I.; Milenkovic, D. Anthocyanins: From sources and bioavailability to cardiovascular-health benefits and molecular mechanisms of action. J. Agric. Food Chem. 2019, 67, 1771-1783. [CrossRef] [PubMed]

5. Jayarathne, S.; Stull, A.J.; Park, O.H.; Kim, J.H.; Thompson, L.; Moustaid-Moussa, N. Protective effects of anthocyanins in obesity-associated inflammation and changes in gut microbiome. Mol. Nutr. Food Res. 2019, 63, e1900149. [CrossRef]

6. Putta, S.; Yarla, N.S.; Peluso, I.; Tiwari, D.K.; Reddy, G.V.; Giri, P.V.; Kumar, N.; Malla, R.; Rachel, V.; Bramhachari, P.V.; et al. Anthocyanins: Multi-target agents for prevention and therapy of chronic diseases. Curr. Pharm. Des. 2017, 23, 6321-6346. [CrossRef]

7. Petroni, K.; Pilu, R.; Tonelli, C. Anthocyanins in corn: A wealth of genes for human health. Planta 2014, 40, 901-911. [CrossRef]

8. Chatham, L.A.; Paulsmeyer, M.; Juvik, J.A. Prospects for economical natural colorants: Insights from maize. Theor. Appl. Genet. 2019, 132, 2927-2946. [CrossRef]

9. Kennett, D.J.; Thakar, H.B.; Van Derwarker, A.M.; Webster, D.L.; Culleton, B.J.; Harper, T.K.; Kistler, L.; Scheffler, T.E.; Hirth, K. High-precision chronology for Central American maize diversification from El Gigante rockshelter, Honduras. Proc. Natl. Acad. Sci. USA 2017, 114, 9026-9031. [CrossRef]

10. Žilić, S.; Serpen, A.; Akıllıŏlu, G.; Gökmen, V.; Vančetović, J. Phenolic compounds, carotenoids, anthocyanins, and antioxidant capacity of colored maize (Zea mays L.) kernels. J. Agric. Food Chem. 2012, 60, 1224-1231. [CrossRef]

11. Lao, F.; Giusti, M.M. Quantification of purple corn (Zea mays L.) anthocyanins using spectrophotometric and HPLC approaches: Method comparison and correlation. Food Anal. Methods 2016, 9, 1367-1380. [CrossRef]

12. Paulsmeyer, M.; Chatham, L.; Becker, T.; West, M.; West, L.; Juvik, J. Survey of anthocyanin composition and concentration in diverse maize germplasms. J. Agric. Food Chem. 2017, 65, 4341-4350. [CrossRef] [PubMed] 
13. Li, X.; Qian, X.; Lü, X.; Wang, X.; Ji, N.; Zhang, M.; Ren, M. Upregulated structural and regulatory genes involved in anthocyanin biosynthesis for coloration of purple grains during the middle and late grain-filling stages. Plant. Physiol. Biochem. 2018, 130, 235-247. [CrossRef] [PubMed]

14. Fan, X.; Fan, B.; Wang, Y.; Yang, W. Anthocyanin accumulation enhanced in Lc-transgenic cotton wunder light and increased resistance to bollworm. Plant. Biotechnol. Rep. 2016, 10, 1-11. [CrossRef] [PubMed]

15. Ming, H.; Wang, Q.; Wu, W.; Liu, H.; Zheng, L.; Zhang, G. Transcriptome analysis reveals the mechanism of anthocyanidins biosynthesis during grains development in purple corn (Zea mays L.). J. Plant. Physiol. 2020, 257, 153328. [CrossRef] [PubMed]

16. Yonemaru, J.; Miki, K.; Choi, S.; Kiyosawa, A.; Goto, K. A genomic region harboring the Pl1 allele from the Peruvian cultivar JC072A confers purple cob on Japanese flint corn (Zea mays L.). Breed. Sci. 2018, 68, 582-586. [CrossRef]

17. Li, T.; Zhang, W.; Yang, H.; Dong, Q.; Ren, J.; Fan, H.; Zhang, X.; Zhou, Y. Comparative transcriptome analysis reveals differentially expressed genes related to the tissue-specific accumulation of anthocyanins in pericarp and aleurone layer for maize. Sci. Rep. 2019, 9, 2485. [CrossRef]

18. Collison, A.; Yang, L.; Dykes, L.; Murray, S.; Awika, J.M. Influence of genetic background on anthocyanin and copigment composition and behavior during thermoalkaline processing of maize. J. Agric. Food Chem. 2015, 63, 5528-5538. [CrossRef]

19. Hu, X.; Liu, J.; Li, W.; Wen, T.; Li, T.; Guo, X.-B.; Liu, R.H. Anthocyanin accumulation, biosynthesis and antioxidant capacity of black sweet corn (Zea mays L.) during kernel development over two growing seasons. J. Cereal Sci. 2020, 95, 103065.

20. Jing, P.; Noriega, V.; Schwartz, S.J.; Giusti, M.M. Effects of growing conditions on purple corncob (Zea mays L.) anthocyanins. J. Agric. Food Chem. 2007, 55, 8625-8629. [CrossRef]

21. Khampas, S.; Lertrat, K.; Lomthaisong, K.; Simla, S.; Suriharn, B. Effect of location, genotype and their interactions for anthocyanins and antioxidant activities of purple waxy corn cobs. Turk. J. Field Crops 2015, 20, 15-23. [CrossRef]

22. Paucar-Menacho, L.M.; Martínez-Villaluenga, C.; Dueñas, M.; Frias, J.; Peñas, E. Optimization of germination time and temperature to maximize the content of bioactive compounds and the antioxidant activity of purple corn (Zea mays L.) by response surface methodology. LWT-Food Sci. Technol. 2017, 76, 236-244. [CrossRef]

23. Somavat, P.; Kumar, D.; Singh, V. Techno-economic feasibility analysis of blue and purple corn processing for anthocyanin extraction and ethanol production using modified dry grind process. Ind. Crops Prod. 2018, 115, 78-87. [CrossRef]

24. Li, Q.; Somavat, P.; Singh, V.; Chatham, L.; de Mejia, E.G. A comparative study of anthocyanin distribution in purple and blue corn coproducts from three conventional fractionation processes. Food Chem. 2017, 231, 332-339. [CrossRef] [PubMed]

25. Deineka, V.I.; Sidorov, A.N.; Deineka, L.A. Determination of purple corn husk anthocyanins. J. Anal. Chem. 2016, 71, 1145-1150. [CrossRef]

26. Chaiittianan, R.; Sutthanut, K.; Rattanathongkom, A. Purple corn silk: A potential anti-obesity agent with inhibition on adipogenesis and induction on lipolysis and apoptosis in adipocytes. J. Ethnopharmacol. 2017, 201, 9-16. [CrossRef] [PubMed]

27. Sarepoua, E.; Tangwongchai, R.; Suriharn, B.; Lertrat, K. Influence of variety and harvest maturity on phytochemical content in corn silk. Food Chem. 2015, 169, 424-429. [CrossRef]

28. Simla, S.; Boontang, S.; Harakotr, B. Anthocyanin content, total phenolic content, and antiradical capacity in different ear components of purple waxy corn at two maturation stages. Aust. J. Crop. Sci. 2016, 10, 675-682. [CrossRef]

29. Žilić, S.; Janković, M.; Basić, Z.; Vančetović, j.; Maksimović, V. Antioxidant activity, phenolic profile, chlorophyll and mineral matter content of corn silk (Zea mays L.): Comparison with medicinal herbs. J. Cereal Sci. 2016, 69, 363e370. [CrossRef]

30. Kapcum, N.; Uriyapongson, J.; Alli, I.; Phimphilai, S. Anthocyanins, phenolic compounds and antioxidant activities in colored corn cob and colored rice bran. Int. Food Res. J. 2016, 23, 2347-2356.

31. Monroy, Y.M.; Rodrigues, R.A.F.; Sartoratto, A.; Cabral, F.A. Optimization of the extraction of phenolic compounds from purple corn cob (Zea mays L.) by sequential extraction using supercritical carbon dioxide, ethanol and water as solvents. J. Supercrit. Fluids 2016, 116, 10-19.

32. Zhu, F. Anthocyanins in cereals: Composition and health effects. Food Res. Int. 2018, 109, 232-249. [CrossRef] [PubMed]

33. Mora-Rochín, S.; Gaxiola-Cuevas, N.; Gutiérrez-Uribec, J.A.; Milán-Carrillo, J.; Milán-Noris, M.; Reyes-Moreno, C.; Serna-Saldivar, S.O.; Cuevas-Rodríguez, E.O. Effect of traditional nixtamalization on anthocyanin content and profile in Mexican blue maize (Zea mays L.) landraces. LWT-Food Sci. Technol. 2016, 68, 563e569. [CrossRef]

34. González-Manzano, S.; Pérez-Alonso, J.J.; Salinas-Moreno, Y.; Mateus, N.; Silva, A.M.S.; de Freitas, V.; Santos-Buelga, C. Flavanol— anthocyanin pigments in corn: NMR characterisation and presence in different purple corn varieties. J. Food Compos. Anal. 2008, 21, 521-526. [CrossRef]

35. Fanzone, M.; González-Manzano, S.; Pérez-Alonso, J.; Escribano-Bailón, M.T.; Jofré, V.; Assof, M.; Santos-Buelga, C. Evaluation of dihydroquercetin-3-O-glucoside from malbec grapes as copigment of malvidin-3-O-glucoside. Food Chem. 2015, 75, 166-173. [CrossRef] [PubMed]

36. Takahama, U.; Yamauchi, R.; Hirota, S. Isolation and characterization of a cyanidin-catechin pigment from adzuki bean (Vigna angularis). Food Chem. 2013, 141, 282-288. [CrossRef] [PubMed]

37. Chatham, L.A.; West, L.; Berhow, M.A.; Vermillion, K.E.; Juvik, J.A. Unique flavanol-anthocyanin condensed forms in Apache red purple corn. J. Agric. Food Chem. 2018, 66, 10844-10854. [CrossRef]

38. Loarca- Piña, G.; Neri, M.; de Dios Figueroa, J.; Castaño-Tostado, E.; Ramos-Gómez, M.; Reynoso, R.; Mendoza, S. Chemical characterization, antioxidant and antimutagenic evaluations of pigmented corn. Food Sci. Technol. 2019, 56, 3177-3184. [CrossRef] 
39. Cuevas Montilla, E.; Hillebrand, S.; Antezana, A.; Winterhalter, P. Soluble and bound phenolic compounds in different bolivian purple corn (Zea mays L.) cultivars. J. Agric. Food Chem. 2011, 59, 7068-7074. [CrossRef]

40. Urias-Lugo, D.A.; Heredia, J.B.; Serna-Saldivar, S.O.; Muy-Rangel, M.D.; Valdez-Torres, J.B. Total phenolics, total anthocyanins and antioxidant capacity of native and elite blue maize hybrids (Zea mays L.). CyTA-J. Food 2014, 13, 336-339. [CrossRef]

41. Nankar, A.; Grant, L.; Scott, P.; Pratt, R.C. Agronomic and kernel compositional traits of blue maize landraces from the Southwestern United States. Crop. Sci. 2016, 56, 2663-2674. [CrossRef]

42. Nankar, A.N.; Dungan, B.; Paz, N.; Sudasinghe, N.; Schaub, T.; Holguin, F.O.; Pratta, R.C. Quantitative and qualitative evaluation of kernel anthocyanins from southwestern United States blue corn. J. Sci. Food Agric. 2016, 96, 4542-4552. [CrossRef] [PubMed]

43. Mutlu, C.; Arslan-Tontul, S.; Candal, C.; Kilic, O.; Erbas, M. Physicochemical, thermal, and sensory properties of blue corn (Zea mays L.). J. Food Sci. 2018, 83, 53-59. [CrossRef] [PubMed]

44. Lago, C.; Landoni, M.; Cassani, E.; Cantaluppi, E.; Doria, E.; Nielsen, E.; Giorgi, A.; Pilu, R. Study and characterization of an ancient European flint white maize rich in anthocyanins: Millo Corvo from Galicia. PLoS ONE 2015, $10, \mathrm{e} 0126521$.

45. Bernardi, J.; Stagnati, L.; Lucini, L.; Rocchetti, G.; Lanubile, A.; Cortellini, C.; De Poli, G.; Busconi, M.; Marocco, A. Phenolic profile and susceptibility to fusarium infection of pigmented maize cultivars. Front. Plant. Sci. 2018, 14, 1189. [CrossRef] [PubMed]

46. Cassani, E.; Puglisi, D.; Cantaluppi, E.; Landoni, M.; Giupponi, L.; Giorgi, A.; Pilu, R. Genetic studies regarding the control of seed pigmentation of an ancient European pointed maize (Zea mays L.) rich in phlobaphenes: The "Nero Spinoso" from the Camonica valley. Genet. Resour. Crop. Evol. 2017, 64, 761-773. [CrossRef]

47. Capocchi, A.; Bottega, S.; Spanò, C.; Fontanini, D. Phytochemicals and antioxidant capacity in four Italian traditional maize (Zea mays L.) varieties. Int. J. Food Sci. Nutr. 2017, 68, 515-524. [CrossRef]

48. Khamphasan, P.; Lomthaisong, K.; Harakotr, B.; Ketthaisong, D.; Scott, M.P.; Lertrat, K.; Suriharn, B. Genotypic variation in anthocyanins, phenolic compounds, and antioxidant activity in cob and husk of purple field corn. Agronomy 2018, 8, 271. [CrossRef]

49. Zhirkova, E.V.; Skorokhodova, M.V.; Martirosyan, V.V.; Sotchenko, E.F.; Malkina, V.D.; Shatalova, T.A. Chemical composition and antioxidant activity of corn hybrids grain of different pigmentation. Foods Raw Mater. 2016, 4, 85-91. [CrossRef]

50. Hong, H.T.; Netzel, M.E.; O'Hare, T.J. Optimisation of extraction procedure and development of LC-DAD-MS methodology for anthocyanin analysis in anthocyanin-pigmented corn kernels. Food Chem. 2020, 319, 126515. [CrossRef]

51. Lao, F.; Giusti, M.M. Extraction of purple corn (Zea mays L.) cob pigments and phenolic compounds using food-friendly solvents. J. Cereal Sci. 2018, 80, 87-93. [CrossRef]

52. Kurambhatti, C.; Kumar, D.; Rausch, K.D.; Tumbleson, M.E.; Singh, V. Improving technical and economic feasibility of water based anthocyanin recovery from purple corn using staged extraction approach. Ind. Crops Prod. 2020, 158, 112976. [CrossRef]

53. Chen, L.; Yang, M.; Mou, H.; Kong, Q. Ultrasound-assisted extraction and characterization of anthocyanins from purple corn bran. Food Process. Preserv. 2018, 42, e13377. [CrossRef]

54. Hernández, M.; Ventura, J.; Castro, C.; Boone, V.; Rojas, R.; Ascacio-Valdés, J.; Martínez-Ávila, G. UPLC-ESI-QTOF-MS2-based identification and antioxidant activity assessment of phenolic compounds from red corn cob (Zea mays L.). Molecules 2018, 23, 1425. [CrossRef] [PubMed]

55. Fernandez-Aulis, F.; Hernandez-Vazquez, L.; Aguilar-Osorio, G.; Arrieta-Baez, D.; Navarro-Ocana, A. Extraction and identification of anthocyanins in corn cob and corn husk from Cacahuacintle maize. J. Food Sci. 2019, 84, 954-962. [CrossRef] [PubMed]

56. Muangrat, R.; Saengcharoenrat, P. Effect of processing conditions of hot pressurized solvent extraction in batch reactor on anthocyanins of purple field corn. Agric. Eng. Int. CIGR J. 2018, 20, 173-182.

57. Piyapanrungrueang, W.; Chantrapornchai, W.; Haruthaithanasan, V.; Sukatta, U.; Aekatasanawan, C. Comparison of anthocyanin extraction methods from high anthocyanin purple corn cob hybrid: Kpsc 901, and quality of the extract powder. J. Food Process. Preserv. 2016, 40, 1125-1133. [CrossRef]

58. Li, Q.; Singh, V.; de Mejia, E.G.; Somavat, P. Effect of sulfur dioxide and lactic acid in steeping water on the extraction of anthocyanins and bioactives from purple corn pericarp. Cereal Chem. 2019, 96, 575-589. [CrossRef]

59. Gu, X.; Cai, W.; Fan, Y.; Ma, Y.; Zhao, X.; Zhang, C. Estimating foliar anthocyanin content of purple corn via hyperspectral model. Food Sci. Nutr. 2018, 6, 572-578. [CrossRef]

60. Saikaew, K.; Lertrat, K.; Meenune, M.; Tangwongchai, R. Effect of high-pressure processing on colour, phytochemical contents and antioxidant activities of purple waxy corn (Zea mays L. var. ceratina) kernels. Food Chem. 2018, 243, 328-337. [CrossRef]

61. Hwang, H.S.; Kùwon, H.S.; Wang, Z.; Kim, H.T.; Kang, Y.-H.; Lee, J.-Y.; Lim, S.S. Optimization of extraction parameters of PTP1 $\beta$ (protein tyrosine phosphatase $1 \beta$ ), inhibitory polyphenols, and anthocyanins from Zea mays L. using response surface methodology (RSM). BMC Complement. Altern. Med. 2016, 16, 317. [CrossRef]

62. Jiang, T.; Zhan, S.; Li, S.; Zhu, Z.; He, J.; Lorenzo, J.M.; Barba, F.J. From 'green' technologies to 'red' antioxidant compounds extraction of purple corn: A combined ultrasound-ultrafiltration-purification approach. J. Sci. Food Agric. 2018, 98, 4919-4927. [CrossRef] [PubMed]

63. Muangrat, R.; Pongsirikul, I.; Blanco, P.H. Ultrasound assisted extraction of anthocyanins and total phenolic compounds from dried cob of purple waxy corn using response surface methodology. J. Food Process. Preserv. 2018, 42, e13447. [CrossRef]

64. Schreiber, H.D.; Swink, A.M.; Godsey, T.D. The chemical mechanism for $\mathrm{Al}^{3+}$ complexing with delphinidin: A model for the bluing of hydrangea sepals. J. Inorg. Biochem. 2010, 104, 732-739. [CrossRef] [PubMed] 
65. Sigurdson, G.T.; Robbins, R.J.; Collins, T.M.; Giusti, M.M. Evaluating the role of metal ions in the bathochromic and hyperchromic responses of cyanidin derivatives in acidic and alkaline pH. Food Chem. 2016, 208, 26-34. [CrossRef] [PubMed]

66. Di Meo, F.; Sancho Garcia, J.C.; Dangles, O.; Trouillas, P. Highlights on anthocyanin pigmentation and copigmentation: A matter of flavonoid pistacking complexation to be described by DFT-D. J. Chem. Theory Comput. 2012, 8, 2034-2043. [CrossRef]

67. Sigurdson, G.T.; Robbins, R.J.; Collins, T.M.; Giusti, M.M. Spectral and colorimetric characteristics of metal chelates of acylated cyanidin derivatives. Food Chem. 2017, 221, 1088-1095. [CrossRef]

68. Jiao, Y.; Zhang, Y.; He, Z.; Zhai, W.; Gong, H.; Yang, Z. Effect of ferulic acid on the formation of pyranoanthocyanins from purple corn (Zea mays L.) cob in a model system and their effects on color. Int. J. Food Prop. 2016, 19, 847-858. [CrossRef]

69. Beta, T.; Hwanga, T. Influence of heat and moisture treatment on carotenoids, phenolic content, and antioxidant capacity of orange maize flour. Food Chem. 2018, 246, 58-64. [CrossRef]

70. Luna-Vital, D.; Li, Q.; West, L.; West, M.; de Mejia, E.G. Anthocyanin condensed forms do not affect color or chemical stability of purple corn pericarp extracts stored under different pHs. Food Chem. 2017, 232, 639-647. [CrossRef]

71. Luna-Vital, D.; Cortez, R.; Ongkowijoyo, P.; de Mejia, E.G. Protection of color and chemical degradation of anthocyanin from purple corn (Zea mays L.) by zinc ions and alginate through chemical interaction in a beverage model. Food Res. Int. 2018, 105, 169-177. [CrossRef]

72. Lao, F.; Giusti, M.M. The effect of pigment matrix, temperature and amount of carrier on the yield and final color properties of spray dried purple corn (Zea mays L.) cob anthocyanin powders. Food Chem. 2017, 227, 376-382. [CrossRef] [PubMed]

73. Ćujić, N.; Trifković, K.; Bugarski, B.; Ibrić, S.; Pljevljakušić, D.; Šavikin, K. Chokeberry (Aronia melanocarpa L.) extract loaded in alginate and alginate/inulinsystem. Ind. Crops Prod. 2016, 86, 120-131. [CrossRef]

74. Aizpurua-Olaizola, O.; Navarro, P.; Vallejo, A.; Olivares, M.; Etxebarria, N.; Usobiaga, A. Microencapsulation and storage stability of polyphenols from Vitis vinifera grape wastes. Food Chem. 2016, 190, 614-621. [CrossRef] [PubMed]

75. Guo, J.; Giusti, M.M.; Kaletunç, G. Encapsulation of purple corn and blueberry extracts in alginate-pectin hydrogel particles: Impact of processing and storage parameters on encapsulation efficiency. Food Res. Int. 2018, 107, 414-422. [CrossRef]

76. Kapcum, C.; Uriyapongson, J. Effects of storage conditions on phytochemical and stability of purple corn cob extract powder. Food Sci. Technol. 2018, 38, 301-305. [CrossRef]

77. Haggard, S.; Luna-Vital, D.; West, L.; Juvik, J.A.; Chatham, L.; Paulsmeyer, M.; de Mejia, E.G. Comparison of chemical, color stability, and phenolic composition from pericarp of nine colored corn unique varieties in a beverage model. Food Res. Int. 2018, 105, 286-297. [CrossRef]

78. Ferron, L.; Colombo, R.; Mannucci, B.; Papetti, A. A new italian purple corn variety (Moradyn) byproduct extract: Antiglycative and hypoglycemic in vitro activities and preliminary bioaccessibility studies. Molecules 2020, 25, 1958. [CrossRef]

79. Huang, B.; Wang, Z.; Park, J.H.; Ryu, O.H.; Choi, M.K.; Lee, J.-Y.; Kang, Y.-H.; Lim, S.S. Anti-diabetic effect of purple corn extract on C57BL/KsJ db/db mice. Nut. Res. Pract. 2015, 9, 22-29. [CrossRef]

80. Luna-Vital, D.; Weiss, M.; de Mejia, E.G. Anthocyanins from purple corn ameliorated tumor necrosis factor- $\alpha$-induced inflammation and insulin resistance in 3T3-L1 adipocytes via activation of insulin signaling and enhanced GLUT4 translocation. Mol. Nutr. Food Res. 2017, 61, 1700362. [CrossRef]

81. Luna-Vital, D.A.; de Mejia, E.G. Anthocyanins from purple corn activate free fatty acid-receptor 1 and glucokinase enhancing in vitro insulin secretion and hepatic glucose uptake. PLOS ONE 2018, 13, e0200449. [CrossRef]

82. Zhang, Q.; de Mejia, E.G.; Luna-Vital, D.; Tao, T.; Chandrasekaran, S.; Chatham, L.; Juvik, J.; Singh, V.; Kumar, D. Relationship of phenolic composition of selected purple maize (Zea mays L.) genotypes with their anti-inflammatory, anti-adipogenic and anti-diabetic potential. Food Chem. 2019, 289, 739-750. [CrossRef] [PubMed]

83. Harakotr, B.; Suriharn, B.; Tangwongchai, R.; Scott, M.P.; Lertrat, K. Anthocyanin, phenolics and antioxidant activity changes in purple waxy corn as affected by traditional cooking. Food Chem. 2014, 164, 510-517. [CrossRef] [PubMed]

84. Mazewski, C.; Liang, K.; de Mejia, E.G. Inhibitory potential of anthocyanin-rich purple and red corn extracts on human colorectal cancer cell proliferation in vitro. J. Funct. Foods 2017, 34, 254-265. [CrossRef]

85. Chen, C.; Somavat, P.; Singh, V.; de Mejia, E.G. Chemical characterization of proanthocyanidins in purple, blue, and red maize coproducts from different milling processes and their anti-inflammatory properties. Ind. Crops Prod. 2017, 109, 464-475. [CrossRef]

86. Damián-Medina, K.; Salinas-Moreno, Y.; Milenkovic, D.; Figueroa-Yáñez, L.; Marino-Marmolejo, E.; Higuera-Ciapara, I.; VallejoCardona, A.; Lugo-Cervantes, E. In silico analysis of antidiabetic potential of phenolic compounds from blue corn (Zea mays L.) and black bean (Phaseolus vulgaris L.). Heliyon 2020, 6, e03632. [CrossRef] [PubMed]

87. Herrera-Sotero, M.Y.; Cruz-Hernández, C.D.; Trujillo-Carretero, C.; Rodríguez-Dorantes, M.; García-Galindo, H.S.; Chávez-Servia, J.L.; Oliart-Ros, R.M.; Guzmán-Gerónimo, R.I. Antioxidant and antiproliferative activity of blue corn and tortilla from native maize. Chem. Cent. J. 2017, 11, 110. [CrossRef]

88. Vázquez-Carrillo, M.G.; Aparicio-Eusebio, L.A.; Salinas-Moreno, Y.; Buendía-Gonzalez, M.O.; Santiago-Ramos, D. Nutraceutical, physicochemical, and sensory properties of blue corn polvorones, a traditional flour-based confectionery. Plant. Foods Hum. Nutr. 2018, 73, 321-327. [CrossRef]

89. Wu, T.; Guo, X.; Zhang, M.; Yang, L.; Liu, R.; Yin, J. Anthocyanins in black rice, soybean and purple corn increase fecal butyric acid and prevent liver inflammation in high fat diet-induced obese mice. Food Funct. 2017, 8, 3178-3186. [CrossRef]

90. Gálvez Ranilla, L.; Christopher, A.; Sarkar, D.; Shetty, K.; Chirinos, R.; Campos, D. Antimicrobial activity of free and bound phenolic fractions from a Peruvian purple corn (Zea mays L.) accession. J. Food Sci. 2017, 82, 2968-2976. [CrossRef] 
91. Trehan, S.; Singh, N.; Kaur, A. Characteristics of white, yellow, purple corn accessions: Phenolic profile, textural, rheological properties and muffin making potential. J. Food Sci. Technol. 2018, 55, 2334-2343. [CrossRef]

92. Hwang, T.; Ndolo, V.U.; Katundu, M.; Nyirenda, B.; Bezner-Kerr, R.; Arntfield, S.; Beta, T. Provitamin A potential of landrace orange maize variety (Zea mays L.) grown in different geographical locations of central Malawi. Food Chem. 2016, 196, 1315-1324. [CrossRef] [PubMed]

93. Lacombe, A.; Wu, V.C.H.; Tyler, S.; Edwards, K. Antimicrobial action of the American cranberry constituents; phenolics, anthocyanins, and organic acids, against Escherichia coli O157:H7. Int. J. Food Microbiol. 2010, 139, 102-107. [CrossRef] [PubMed]

94. Kim, S.H.; Park, M.; Woo, H.; Tharmalingam, N.; Lee, G.; Rhee, K.J.; Eon, Y.B.; Han, S.I.; Seo, W.D.; Kim, J.B. Inhibitory effects of anthocyanins on secretion of Helicobacter pylori CagA and VacA toxins. Int. J. Med. Sci. 2012, 9, 838-842. [CrossRef] [PubMed]

95. Kim, J.M.; Kim, K.M.; Park, E.H.; Seo, J.H.; Song, J.Y.; Shin, S.C.; Kang, H.L.; Lee, W.K.; Cho, M.J.; Rhee, K.H.; et al. Anthocyanins from black soybean inhibit Helicobacter pylori-induced inflammation in human gastric epithelial AGS cells. Microbiol. Immunol. 2013, 57, 366-373. [CrossRef]

96. Syedd-León, R.; Orozco, R.; Álvarez, V.; Carvajal, Y.; Rodríguez, G. Chemical and antioxidant charaterization of native corn germplasm from two regions of Costa Rica: A conservation approach. Int. J. Food Sci. 2020, 2020, 2439541. [CrossRef]

97. Mendoza-Díaz, S.; Ortíz-Valerio Ma, C.; Castaño-Tostado, E.; Figueroa-Cárdenas, J.D.; Reynoso-Camacho, R.; Ramos-Gómez, M.; Loarca-Piña, G. Antioxidant capacity and antimutagenic activity of anthocyanin and carotenoid extracts from nixtamalized pigmented creole maize races (Zea mays L.). Plant. Food Hum. Nutr. 2012, 67, 442-449. [CrossRef]

98. World Health Organization. (2020). Fact Sheet: Diabetes, 15 May 2020

99. World Health Organization. (2020). Fact Sheet: Obesity and Overweight, 1 April 2020

100. Gálvez Ranilla, L.; Huamán-Alvino, C.; Flores-Bâez, O.; Aquino-Méndez, E.M.; Chirinos, R.; Campos, D.; Sevilla, R.; Fuentealba, C.; Pedreschi, R.; Sarkar, D.; et al. Evaluation of phenolic antioxidant-linked in vitro bioactivity of Peruvian corn (Zea mays L.) diversity targeting for potential management of hyperglycemia and obesity. J. Food Sci. Technol. 2019, 56, 2909-2924. [CrossRef]

101. Wojdyło, A.; Nowicka, P.; Carbonell-Barrachina, Á.A.; Hernández, F. Phenolic compounds, antioxidant and antidiabetic activity of different cultivars of Ficus carica L. fruits. J. Funct. Foods 2016, 25, 421-432. [CrossRef]

102. Ademiluyi, A.O.; Oboh, G.J.E. Soybean phenolic-rich extracts inhibit key enzymes linked to type 2 diabetes ( $\alpha$-amylase and $\alpha$-xglucosidase) and hypertension (angiotensin I converting enzyme) in vitro. Exp. Toxicol. Pathol. 2013, 65, 305-309. [CrossRef]

103. Bhaswant, M.; Shafie, S.R.; Mathai, M.L.; Mouatt, P.; Brown, L. Anthocyanins in chokeberry and purple maize attenuate diet-induced metabolic syndrome in rats. Nutrition 2017, 41, 24-31. [CrossRef]

104. Guzmán-Gerónimo, R.I.; Alarcón-Zavaleta, T.M.; Oliart-Ros, R.M.; Meza-Alvarado, J.E.; Herrera-Meza, S.; Chávez-Servia, J.L. Blue maize extract improves blood pressure, lipid profiles, and adipose tissue in high-sucrose diet-induced metabolic syndrome in rats. J. Med. Food 2017, 20, 110-115. [CrossRef] [PubMed]

105. Reyna Gallegos, S.; Torres Arrunátegui, G.; Valenzuela, R.; Rincón-Cervera, M.Á.; Villanueva Espinoza, M.E. Adding a purple corn extract in rats supplemented with chia oil decreases gene expression of SREBP-1c and retains $\Delta 5$ and $\Delta 6$ hepatic desaturase activity, unmodified the hepatic lipid profile. Prostaglandins, Leukot. Essent. Fatty Acids 2018, 132, 1-7. [CrossRef] [PubMed]

106. Wattanathorn, J.; Thiraphatthanavong, P.; Muchimapura, S.; Thukhammee, W.; Lertrat, K.; Suriharn, B. The combined extract of Zingiber officinale and Zea mays (Purple Color) improves neuropathy, oxidative stress, and axon density in streptozotocin induced diabetic rats. Evid. Based Complement. Alternat. Med. 2015, 2015, 301029. [CrossRef] [PubMed]

107. Zhang, Q.; Luna-Vital, D.; de Mejia, E.G. Anthocyanins from colored maize ameliorated the inflammatory paracrine interplay between macrophages and adipocytes through regulation of NF-kB and JNK-dependent MAPK pathways. J. Funct. Foods 2019, 54, 175-186. [CrossRef]

108. Magaña-Cerino, J.M.; Tiessen, A.; Soto-Luna, I.C.; Peniche-Pavía, H.A.; Vargas-Guerrero, B.; Domínguez-Rosales, J.A.; GarcíaLópez, P.M.; Gurrola-Díaz, C.M. Consumption of nixtamal from a new variety of hybrid blue maize ameliorates liver oxidative stress and inflammation in a high-fat diet rat model. J. Funct. Foods 2020, 72, 104075. [CrossRef]

109. Hosoda, K.; Miyaji, M.; Matsuyama, H.; Haga, S.; Ishizaki, H.; Nonaka, K. Effect of supplementation of purple pigment from anthocyanin-rich corn (Zea mays L.) on blood antioxidant activity and oxidation resistance in sheep. Livest. Sci. 2012, 145, 266-270. [CrossRef]

110. Mazza, G.; Kay, C.D.; Cottrell, T.; Holub, B.J. Absorption of anthocyanins from blueberries and serum antioxidant status in human subjects. J. Agric. Food Chem. 2002, 50, 7731-7737. [CrossRef]

111. Matsuba, T.; Kubozono, H.; Saegusa, A.; Obata, K.; Gotoh, K.; Miki, K.; Akiyama, T.; Oba, M. Short communication: Effects of feeding purple corn (Zea mays L.) silage on productivity and blood superoxide dismutase concentration in lactating cows. J. Dairy Sci. 2019, 102, 7179-7182.

112. Tian, X.; Xin, H.; Paengkoum, P.; Paengkoum, S.; Ban, C.; Sorasak, T. Effects of anthocyanin-rich purple corn (Zea mays L.) stover silage on nutrient utilization, rumen fermentation, plasma antioxidant capacity, and mammary gland gene expression in dairy goats. J. Anim. Sci. 2019, 97, 1384-1397. [CrossRef]

113. Tian, X.Z.; Paengkoum, P.; Paengkoum, S.; Chumpawadee, S.; Ban, C.; Thongpea, S. Short communication: Purple corn (Zea mays L.) stover silage with abundant anthocyanins transferring anthocyanin composition to the milk and increasing antioxidant status of lactating dairy goats. J. Dairy Sci. 2019, 102, 413-418. [CrossRef]

114. Aguirre López, L.O.; Chávez Servia, J.L.; Gómez Rodiles, C.C.; Beltrán Ramírez, J.R.; Bañuelos Pineda, J. Blue corn tortillas: Effects on learning and spatial memory in rats. Plant Foods Hum. Nutr. 2017, 72, 448-450. [CrossRef] [PubMed] 
115. Kirisattayakul, W.; Wattanathorn, J.; Iamsaard, S.; Jittiwat, J.; Suriharn, B.; Lertrat, K. Neuroprotective and memory-enhancing effect of the combined extract of purple waxy corn cob and pandan in ovariectomized rats. Oxid. Med. Cell. Longev. 2017, 2017, 5187102. [CrossRef] [PubMed]

116. Wattanathorn, J.; Kirisattayakul, W.; Suriharn, B.; Lertrat, K. Functional drink containing the extracts of purple corn cob and pandan leaves, the novel cognitive enhancer, increases spatial memory and hippocampal neuron density through the improvement of extracellular signal regulated protein kinase expression, cholinergic function, and oxidative status in ovariectomized rats. Rejuvenation Res. 2018, 21, 431-441.

117. Intuyod, K.; Priprem, A.; Pairojkul, C.; Hahnvajanawong, C.; Vaeteewoottacharn, K.; Pinlaor, P.; Pinlaor, S. Anthocyanin complex exerts anti-cholangiocarcinoma activities and improves the efficacy of drug treatment in a gemcitabine-resistant cell line. Int. J. Oncol. 2018, 52, 1715-1726. [CrossRef] [PubMed]

118. Magni, G.; Marinelli, A.; Riccio, D.; Lecca, D.; Tonelli, C.; Abbracchio, M.P.; Petroni, K.; Ceruti, S. Purple corn extract as anti-allodynic treatment for trigeminal pain: Role of microglia. Fron. Cell. Neurosci. 2018, 12, 378. [CrossRef]

119. Wiraguna, A.A.G.P.; Dianasari, R.; Pangkahila, W. The topical skin application of purple corn extract (zea mays) inhibited the increase in mmp-1 levels and decreased collagen in wistar rats (rattus norvegicus) exposed to uv-b rays. Biomed. Pharmacol. J. 2019, 12, 297-304. [CrossRef]

120. Priprem, A.; Damrongrungruang, T.; Limsitthichaikoon, S.; Khampaenjiraroch, B.; Nukulkit, C.; Thapphasaraphong, S.; Limphirat, W. Topical niosome gel containing an anthocyanin complex: A potential oral wound healing in rats. AAPS Pharm. Sci. Tech. 2018, 19, 1681-1692. [CrossRef]

121. Petroni, K.; Trinei, M.; Fornari, M.; Calvenzani, V.; Marinelli, A.; Micheli, L.A.; Pilu, R.; Matros, A.; Mock, H.-P.; Tonelli, C.; et al. Dietary cyanidin 3-glucoside from purple corn ameliorates doxorubicin-induced cardiotoxicity in mice. Nutr. Metab. Cardiovasc. Dis. 2017, 27, 462-469. [CrossRef]

122. Carro-Juárez, M.; Rodríguez-Santiago, M.G.; Franco, M.A.; Hueletl-Soto, M.E. Aphrodisiac activity of the aqueous crude extract of purple corn (Zea mays) in male rats. J. Evid. Based Complementary Altern. Med. 2017, 22, 637-645. [CrossRef]

123. Villasante, A.; Patro, B.; Chew, B.; Becerra, M.; Wacyk, J.; Overturf, K. Dietary intake of purple corn extract reduces fat body contentand improves antioxidant capacity and n-3 polyunsaturated fatty acid profile in plasma of rainbow trout, Oncorhynchus mykiss. J. World Aquac. Soc. 2015, 46, 381-393. [CrossRef]

124. Moreira, R.; Chenlo, F.; Arufe, S.; Rubinos, S.N. Physicochemical characterization of white, yellow and purple maize flours and rheological characterization of their doughs. Food Sci. Technol. 2015, 52, 7954-7963. [CrossRef] [PubMed]

125. Sebastián Mansilla, P.; Nazar, M.C.; Pérez, G.T. Flour functional properties of purple maize (Zea mays L.) from Argentina. Influence of environmental growing conditions. Int. J. Biol. Macromol. 2020, 146, 311-319. [CrossRef] [PubMed]

126. Camelo-Méndez, G.A.; Tovar, J.; Bello-Pérez, L.A. Influence of blue maize flour on gluten-free pasta quality and antioxidant retention characteristics. J. Food Sci. Technol. 2018, 55, 2739-2748. [CrossRef] [PubMed]

127. Navarro Cortez, R.O.; Gómez-Aldapa, C.A.; Aguilar-Palazuelos, E.; Delgado-Licon, E.; Castro Rosas, J.; Hernández-Ávila, J.; Solís-Soto, A.; Ochoa-Martínez, A.; Medrano-Roldána, H. Blue corn (Zea mays L.) with added orange (Citrus sinensis) fruit bagasse: Novel ingredients for extruded snacks. CyTA-J. Food 2016, 14, 349-358. [CrossRef]

128. Mariscal Moreno, R.M.; Figuero, J.D.C.; Santiago-Ramos, D.; Arámbula Villa, G.; Sandoval, S.J.; Rayas-Duarte, P.; Véles-Medina, J.J.; Martínez Flores, H.E. The effect of different nixtamalisation processes on some physicochemical properties, nutritional composition and glycemic index. J. Cereal Sci. 2015, 65, 140-146. [CrossRef]

129. Escalante-Aburto, A.; Mariscal-Moreno, R.M.; Santiago-Ramos, D.; Ponce-García, N. An update of different nixtamalization technologies, and its effects on chemical composition and nutritional value of corn tortillas. Food Rev. Int. 2020, 36, 456-498. [CrossRef]

130. Santiago-Ramos, D.; de Dios Figueroa-Cárdenas, J.; Mariscal-Moreno, R.M.; Escalante-Aburto, A.; Ponce-García, N.; Véles-Medina, J.J. Physical and chemical changes undergone by pericarp and endosperm during corn nixtamalization-A review. J. Cereal Sci. 2018, 81, 108-117. [CrossRef]

131. Chimimba, J.; Pratt, R.; Cuellar, M.; Delgado, E. Quality parameters of masa and tortillas produced from blue maize (Zea mays sp. mays) landraces. J. Food Sci. 2019, 84, 213-223. [CrossRef]

132. Corrales-Bañuelos, A.B.; Cuevas-Rodríguez, E.O.; Gutiérrez-Uribe, J.A.; Milán-Noris, E.M.; Reyes-Moreno, C.; Milán-Carrillo, J.; Mora-Rochín, S. Carotenoid composition and antioxidant activity of tortillas elaborated from pigmented maize landrace by traditional nixtamalization or lime cooking extrusion process. J. Cereal Sci. 2016, 69, 64-70. [CrossRef]

133. Colín-Chávez, C.; Virgen-Ortiz, J.J.; Serrano-Rubio, L.E.; Martínez-Téllez, M.A.; Astier, M. Comparison of nutritional properties and bioactive compounds between industrial and artisan fresh tortillas from maize landraces. Curr. Res. Food Sci. 2020, 3, 189-194. [CrossRef]

134. Amador-Rodríguez, K.Y.; Pérez-Cabrera, L.E.; Guevara-Lara, F.; Chávez-Vela, N.A.; Posadas-Del Río, F.A.; Silos-Espino, H.; Martínez-Bustos, F. Physicochemical, thermal, and rheological properties of nixtamalized blue corn flours and masas added with huitlacoche (Ustilago maydis) paste. Food Chem. 2019, 278, 601-608. [CrossRef] [PubMed]

135. Kokkaew, H.; Pitirit, T. Optimization for anthocyanin and antioxidant contents and effects of acidulants on purple corn cake containing corn silk powder qualities. Int. Food Res. J. 2016, 23, 2390-2398.

136. Flores-Calderón, A.M.D.; Luna, H.; Escalona-Buendía, H.B.; Verde-Calvo, J.R. Chemical characterization and antioxidant capacity in blue corn (Zea mays L.) malt beers. J. Inst. Brew. 2017, 123, 506-518. [CrossRef] 
137. Salvador-Reyes, R.; Pedrosa Silva Clerici, M.T. Peruvian Andean maize: General characteristics, nutritional properties, bioactive compounds, and culinary uses. Food Res. Int. 2020, 130, 108934. [CrossRef] [PubMed]

138. Tian, X.Z.; Lu, Q.; Paengkoum, P.; Paengkoum, S. Short communication: Effect of purple corn pigment on changeof anthocyanin composition and unsaturated fatty acids during milk storage. J. Dairy Sci. 2020, 103, 7808-7812. [CrossRef]

139. Camelo-Méndez, G.A.; Agama-Acevedo, E.; Sanchez-Rivera, M.M.; Bello-Pérez, L.A. Effect on in vitro starch digestibility of Mexican blue maize anthocyanins. Food Chem. 2016, 211, 281-284. [CrossRef]

140. Rocchetti, G.; Giuberti, G.; Gallo, A.; Bernardi, J.; Marocco, A.; Lucini, L. Effect of dietary polyphenols on the in vitro starch digestibility of pigmented maize varieties under cooking conditions. Food Res. Int. 2018, 108, 183-191. [CrossRef]

141. Wang, Z.; Huang, H.; de Mejia, E.G.; Li, Q.; Singh, V. Use of pigmented maize in both conventional dry-grind and modified processes using granular starch hydrolyzing enzyme. Cereal Chem. 2016, 93, 344-351. [CrossRef]

142. Somavat, P.; Li, Q.; de Mejia, E.G.; Liu, W.; Singh, V. Coproduct yield comparisons of purple, blue and yellow dent corn for various milling processes. Ind. Crops Prod. 2016, 87, 266-272. [CrossRef]

143. Qin, Y.; Liu, Y.; Yuan, L.; Yong, H.; Liu, J. Preparation and characterization of antioxidant, antimicrobial and $\mathrm{pH}$ sensitive films based on chitosan, silver nanoparticles and purple corn extract. Food Hydrocoll. 2019, 96, 102-111. [CrossRef]

144. Phinjaturus, K.; Maiaugree, W.; Suriharn, B.; Pimanpaeng, S.; Amornkitbamrung, V.; Swatsitang, E. Dye-sensitized solar cells based on purple corn. Appl. Surf. Sci. 2016, 380, 101-107. [CrossRef] 